\title{
The streptococcal collagen-like protein-1 (Scl1) is a multifunctional adhesin in extracellular matrix interactions, biofilm formation, and host colonization by group A Streptococcus
}

Heaven A. Oliver-Kozup

West Virginia University

Follow this and additional works at: https://researchrepository.wvu.edu/etd

\section{Recommended Citation}

Oliver-Kozup, Heaven A., "The streptococcal collagen-like protein-1 (Scl1) is a multifunctional adhesin in extracellular matrix interactions, biofilm formation, and host colonization by group A Streptococcus" (2013). Graduate Theses, Dissertations, and Problem Reports. 570.

https://researchrepository.wvu.edu/etd/570

This Dissertation is protected by copyright and/or related rights. It has been brought to you by the The Research Repository @ WVU with permission from the rights-holder(s). You are free to use this Dissertation in any way that is permitted by the copyright and related rights legislation that applies to your use. For other uses you must obtain permission from the rights-holder(s) directly, unless additional rights are indicated by a Creative Commons license in the record and/ or on the work itself. This Dissertation has been accepted for inclusion in WVU Graduate Theses, Dissertations, and Problem Reports collection by an authorized administrator of The Research Repository @ WVU.

For more information, please contact researchrepository@mail.wvu.edu. 
The streptococcal collagen-like protein-1 (Scl1) is a multifunctional adhesin in extracellular matrix interactions, biofilm formation, and host colonization by group A Streptococcus

Heaven A. Oliver-Kozup

\author{
Dissertation submitted to the \\ School of Medicine \\ at West Virginia University \\ in partial fulfillment of the requirements \\ for the degree of
}

Doctor of Philosophy

in

Immunology and Microbial Pathogenesis

Slawomir Lukomski, Ph.D., Chair

Nyles Charon, Ph.D.

Karen Martin, Ph.D.

Rosana Schafer, Ph.D.

Peter Gannett, Ph.D.

Department of Microbiology, Immunology, and Cell Biology

Morgantown, WV

2013

Keywords: Group A Streptococcus, Scl1, cellular fibronectin, biofilm, wound infection 


\section{Abstract \\ The streptococcal collagen-like protein-1 (Scl1) is a multifunctional adhesin in extracellular matrix interactions, biofilm formation, and host colonization by group A Streptococcus}

\section{Heaven A. Oliver-Kozup}

Group A Streptococcus (GAS) is responsible for over 100 million skin infections annually that may lead to invasive episodes and autoimmune sequelaes. Wounds and unapparent skin infringements are susceptible to the introduction of GAS and serve as a portal of entry. The nutritional nature of a wound and the accessibility to exposed host cell surfaces and extracellular matrix components create a model milieu for successful host colonization and potential biofilm formation. The initiation of biofilm is often mediated by bacterial surface adhesins and has been shown to interfere with wound healing by blocking critical host cellular interactions. However, the underlying molecular mechanisms supporting this impediment of wound healing are not completely understood. Here, we characterize the GAS surface adhesin, streptococcal collagen-like protein-1 (Scl1), and its interaction with extracellular matrix components and contribution to GAS biofilm formation. Scl1 has been detected in all GAS strains and extends from the GAS surface as a homotrimeric protein composed of a collagen-like domain, structurally similar to mammalian collagen, and an adjacent aminoterminal non-collagenous variable domain. The current work (i) identifies that the Scl1 variable domain binds to extracellular matrix proteins, cellular fibronectin and laminin and contributes to GAS adherence and internalization (ii) demonstrates that the Scl1 protein is an important determinant of GAS biofilm formation on untreated and ECM-deposited surfaces, and (iii) characterizes a novel molecular mechanism by which GAS utilizes the Scl1 protein to specifically target the EDA-containing variant of cellular fibronectin predominantly expressed at the site of injured tissue in order to secure host colonization and biofilm formation. In total, these studies further demonstrate the importance of the Scl1 protein in GAS pathogenesis. 


\section{Dedication}

I dedicate this research to my parents, Tony and Phyllis Oliver, who have supported each endeavor throughout my life, given me love and the strength to persevere. They have been my inspiration. Without all you have provided, I would not be here and made it as far as I have. Thank you for having such broad shoulders and always being my biggest fans. This work is also dedicated to my husband, John C. Kozup for his love and support over the last eight years. Thank you for having the courage to help me fulfill my dream and the faith to know that I always could. It means more to me than words can express. I love you all. 


\section{Acknowledgements}

I would like to thank my mentor Dr. Slawomir Lukomski for his continued support and guidance throughout my research. I hope this will always remain. I thank my advisory committee members: Dr. Nyles Charon, Dr. Rosana Schafer, Dr. Karen Martin and Dr. Peter Gannett for their mentorship and friendship. Special thanks go to Dr. Joan Olson and Dr. Fred Minnear for their contribution in pre-publication manuscript evaluation and for career advice. 


\section{Table of Contents}

Content

Page

Abstract

ii

Dedication iii

Acknowledgments iv

Table of Content v

List of Tables vi

List of Figures vii

Chapter 1 - General Introduction 1

Overview of Group A Streptococcus (GAS) 2

Streptococcal collagen-like proteins (Scl) 10

Chapter - 2 Streptococcal collagen-like protein-1 (Scl1) Interacts with Selected Extracellular Matrix Components

Abstract 22

Introduction 23

Materials and methods 25

Results/Discussion 28

Acknowledgments 35

References 36

Figures and figure legends 39

Chapter 3 - Contribution of the Streptococcal collagen-like protein-1 (Scl1) in Biofilm Formation by Group A Streptococcus

Abstract 44 


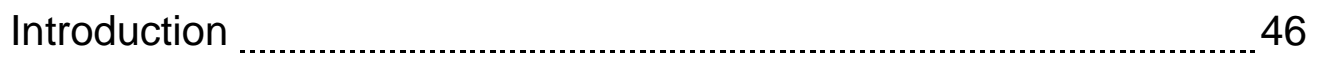

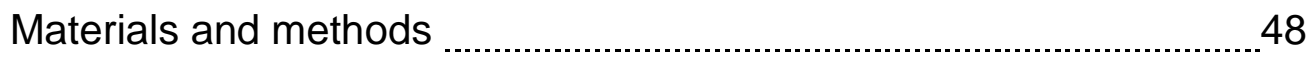

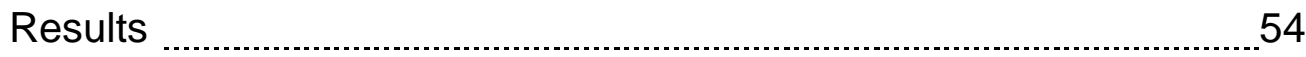

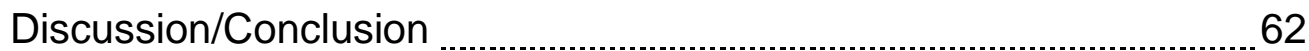

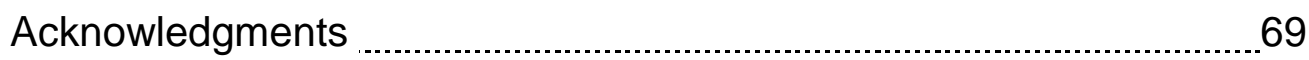

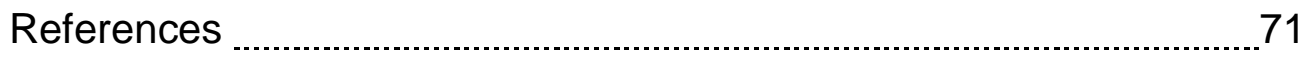

Figures and figure legends .............................................................. 76

Chapter 4 - Streptococcal Collagen-like Protein-1 (Scl1) Targets a Specific Variant of Cellular Fibronectin Expressed in Wounded Tissue ................93

Abstract

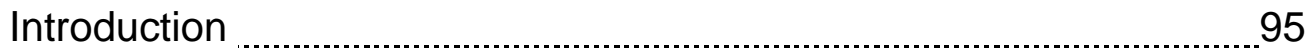

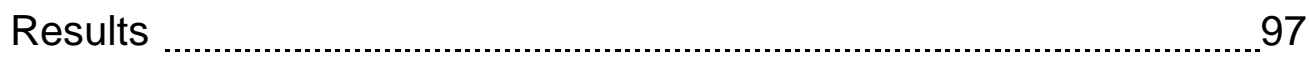

Discussion/Conclusion ............................................................................. 106

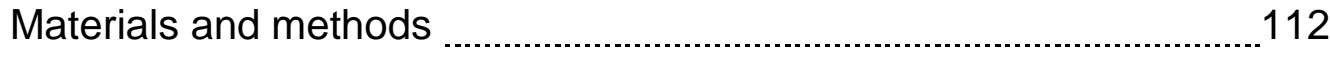

Acknowledgments

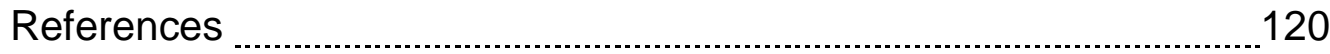

Figures and figure legends ................................................................. 126

Chapter 5 - General Discussion ................................................................ 140

General References

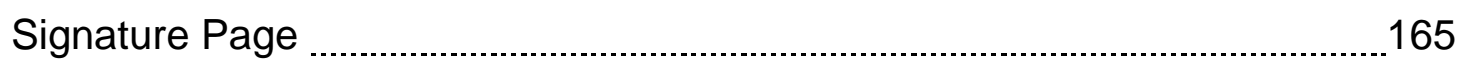




\section{List of Tables}

Chapter/Table

Page

Chapter 1 - General Introduction

Table 1. Group A Streptococcus Fibronectin-Binding Proteins 9

Chapter 3 - Contribution of Streptococcal collagen-like protein-1 (Scl1) in Biofilm Formation by Group A Streptococcus

Table 1. Cell surface hydrophobicity of GAS strains 91

Table 2. Cell surface hydrophobicity of Lactococcus lactis 92 


\section{List of Figures}

Figure

Page

Chapter 1 - General Introduction

Figure 1. Structure and domain organization of Scl1 12

Figure 2. Dichotomous binding model of Scl1 15

Chapter 2. - Streptococcal collagen-like protein-1 (Scl1) Interacts with Selected Extracellular Matrix Components

Figure 1. Characterization of ECM binding to rScl1.41 protein 39

Figure 2. Scl1.41-mediated binding of GAS cells to ECM proteins ....40

Figure 3. Scl1-ECM interactions mediate GAS internalization by human epithelial cells 41

Figure 4. Characterization of ECM binding to rScl proteins 42

Chapter 3 - Contribution of Streptococcal collagen-like protein-1 (Scl1) in Biofilm Formation by Group A Streptococcus

Figure 1. Variation in biofilm formation among GAS strains 76

Figure 2. Field emission electron microscopy of GAS biofilm 77

Figure 3. Production of bacterial-associated extracellular matrix (BAEM) 78

Figure 4. Biofilm formation by wild-type and scl1-inactivated isogenic mutants 79

Figure 5. Scl1 expression in L. lactis promotes biofilm formation .......80

Figure 6. Scl1-mediated GAS biofilm model 81

Supplementary Figure 1 (a-f): Gallery views of GAS biofilm 
Supplementary Figure 2 (a-c): Gallery view of Lactococcus lactis biofilm (CLSM) 88

\section{Chapter 4 - Streptococcal Collagen-like Protein-1 (Scl1) Targets a Specific Variant of Cellular Fibronectin Expressed in Wounded Tissue}

Figure 1. The EDA domain mediates Scl1 binding to cFn 126

Figure 2. Scl1-cFn interaction is mediated by the C-C' loop region of EDA 127

Figure 3. Scl1-EDA interaction represents a common, cFn-binding mechanism among epidemiologically diverse GAS strains 129

Figure 4. Scl1-cFn binding mediates and enhances biofilm formation by GAS

Figure 5. Scl1 binding to ECM derived from human dermal fibroblasts 133

Figure 6. Inhibition of GAS adhesion by targeting the C-C' loop of EDA

Figure 7. A model of Scl1-mediated wound colonization by GAS 137

Supplementary Figure 1: Characterization of fibroblast-derived extracellular matrix ( $\mathrm{fdECM}$ ) 138 Supplementary Figure 2: Adhesion inhibition of GAS scl1 mutants by targeting the C-C' loop of EDA 


\section{Chapter 5: General Introduction}

Figure 1. Step-by-step model of wound healing and role of Scl1 in host

colonization 153

Figure 2. Scl1-mediated GAS biofilm formation model 155 
Chapter 1

GENERAL INTRODUCTION 
Overview of Group A Streptococcus. Streptococcus pyogenes comprises the single group within the Lancefield classification scheme known as group A Streptococcus (GAS), which is based on serologic typing of the cell surface carbohydrate. Strains have been further subtyped based on the surfaceexpressed M-protein (21). GAS is a gram-positive, adaptive, exclusive human pathogen that causes a wide spectrum of diseases but can also reside undetected among asymptomatic human carriers (75), with a rate that can exceed 20 percent in school-age populations. GAS commonly causes throat and skin infections. Suppurative, non-invasive diseases such as pharyngitis and pyoderma make up 75 percent of global GAS cases. GAS is also responsible for severe, life-threatening, invasive diseases such as necrotizing fasciitis and streptococcal toxic-shock syndrome $(82,111,112,123)$. Mounting poststreptococcal diseases account for 11 percent of global GAS-associated cases. Rheumatic heart disease (RHD) and acute glomerulonephritis (AGN) are among these sequelae (42). In addition, research in recent years has linked GAS infections with a range of neurological conditions in children collectively referred to as 'pediatric autoimmune neuropsychiatric disorders associated with streptococcal infections' (PANDAS) $(7,69,74)$. On a positive note, extensive genetic and molecular research on GAS is unyielding, living conditions and nutrition are improving in third-world countries with GAS epidemics, treatment of GAS infections is advancing due to better diagnostic tools, and surveillance and reports of outbreaks occurring are broadening worldwide. However, much is yet to be discovered about the interactions of GAS with the human host. 
GAS Epidemiology and Global Burden. Epidemiology of GAS has progressively changed over the last two hundred years with the improvements in nutritional and environmental conditions contributing. Historically, epidemics have been documented in Europe during early nineteenth and twentieth centuries regarding infections that would resemble current clinical manifestations such as scarlet fever. The continued global prevalence of GAS results in increasing cases of throat and skin diseases, specifically in less developed countries. In the United States, there are 25-35 million cases of GAS suppurative, non-invasive throat and skin infections such as pyoderma and pharyngitis per year, resulting in a financial burden of up to 2 billion dollars annually. Global estimates of throat and skin infections reach above 600 million total cases of GAS infections each year (10). Life-threatening, invasive infections and non-suppurative sequelae account for more than 18 million cases globally, increasing by almost 2 million each year. The mortality rate from acquisition of these diseases is approaching 3 percent or 520,000 annually $(75,78)$. Supporting this degree of prevalence, GAS expresses numerous virulence factors that contribute to its role as a successful human pathogen.

GAS Pathogenesis Strategies and Virulence Factors. Over the last decade, molecular pathogenomics has facilitated and heightened our understanding of genetics and molecular mechanisms contributing to GAS pathogenesis. This broadened spectrum of knowledge allows us to anticipate prospective GAS infections resulting from clone-emergence or previous strain reemergence appearing as an altered clinical phenotype. Coordination of 
secreted and surface expressed virulence factors provides a well-tuned arsenal for host colonization and immune evasion that is reliant upon environmental and growth conditions, phase of growth of the bacterium but also the immune response and genetic predisposition of the host (75). Virulence factors include the cell surface-expressed proteins such as the $M$ protein and streptococcal collagen-like proteins, hyaluronic acid capsule, pili, extracellular matrix binding proteins, proteinases, DNases, and streptokinases. Numerous superantigens have been identified in the GAS genome with several located on mobile DNA elements and bacteriophages (93). Expression profiles of these virulence factors are strain-specific which also produces variations in pathogenesis strategies and disease capacity $(17,120)$. Many genes encoding these virulence factors are located within the mga regulon. Mga, the multigene activator, encoded by the mga gene coordinates the expression of some of these factors, which specifically includes the $M$ protein, in response to environmental stresses and phases of growth (3).

The M-protein is the most abundant fibrillar coiled-coil homodimeric protein appearing as hair-like projections extending from the cell wall. Serological $M$ typing exploiting the hypervariable amino-terminal region of the protein, which displays extensive antigenic heterogeneity, was used historically to distinguish $\mathrm{M}$ serotypes $(31,42)$. This method was replaced with a nucleotide sequence-based typing scheme, which resulted in the identity of more than 200 types now listed online (http://www.cdc.gov/ncidod/biotech/strep/strepindex.htm). Based on the number and arrangement of the emm and emm-like genes, there are five 
conclusive patterns: A-C, D, and E. These patterns serve as reliable, genetic biomarkers for tissue tropism with pattern A-C genotypes showing a strong preference toward throat infections and pattern $D$ genotypes having a predilection for skin infections $(4,5,57)$.

GAS Treatment and Vaccine Development Strategies. In the preantibiotic era, GAS infections were very common and responsible for almost half of post-partum deaths as well as being the main bacterium associated with burn victims (29). After the introduction of penicillin, reports of GAS disease had dramatically declined by the 1970s. However, by the 1990s, resurgence in not only superficial infections but also post-streptococcal sequelae surfaced around the globe (29).

The study of GAS's antigenic variation and mechanisms of immune evasion aid in the development of an efficacious vaccine that addresses both superficial and invasive infections. Potential vaccine candidates most often include conserved cell-surface proteins associated with multiple GAS strains such as the C5a peptidase ( $\operatorname{ScpA})$ which proved to be successful in reducing GAS colonization within a rabbit pharyngitis model $(16,48)$. Other investigations have identified a number of other vaccine candidates including fibronectin binding proteins, F1 and $\mathrm{FBP} 54(19,37,52)$ as well as the group A carbohydrate (136). Unfortunately, efficacy and degree of protection have not been established for these proteins. The development of a multivalent vaccine containing the amino-terminal peptides of $M$ proteins from twenty-six epidemiologically important M-types (68) was completed; however, clinical trials were only in part 
successful in disease inhibition after challenge. Because the majority of GAS infections occur within the mucosal membrane of the nasopharynx, several mucosally-active vaccines, including virus-like particle (VLP) approaches and lipopeptide delivery systems, have been developed which incorporate antigenic peptides derived from the M-protein. Success has been only partial in mouse models. Many disadvantages prevail in developing a universal vaccine for GAS diseases including antigenic variation among the same serotype, cross-reactivity between GAS and human antigens resulting in autoimmune sequelae, and the geographic distribution of strains $(43,100,110,137,138)$. Collective efforts are ongoing to develop an efficacious, non-toxic, cheap, and easily distributable vaccine for GAS.

GAS Interaction with Extracellular Matrix. Bacterial adherence within host tissues is the first and most crucial step in colonization within a host point of entry and mediated by surface adhesins. Many 'microbial surface components recognizing adhesive matrix molecules' (MSCRAMMs) have been identified for GAS and characterized based on their interaction with eukaryotic cell surface components and extracellular matrix components such as collagen, laminin, fibrinogen and elastin. Among the more reported interaction is the binding to fibronectin (Fn). The fibronectins, plasma (soluble) and cellular (insoluble) forms, comprise of large family of glycoproteins that are biologically important in cellular interactions of the extracellular matrix including matrix assembly and restructuring, cell migration, and cell differentiation (87). Fn is a common target for GAS and is used as a "bridging mechanism" for adhesion and internalization 
into epithelial and endothelial cells. The expression profile of these Fn-binding adhesins on the GAS surface varies among the M-types and often provides information toward tissue specificity and type as well as the potential severity of GAS pathogenesis (130). More than eleven Fn-binding proteins have been identified to date that can be grouped into two major categories: those containing Fn-binding repeats and those lacking them. Table 1 lists the reported GAS Fnbinding proteins, including their Fn-binding site (if available) and the resulting function (if known). Of these proteins, the most extensively studied in vitro is the F1 (PrtF1/Sfbl) protein. The importance of this protein in GAS pathogenesis has been widely supported with most of these studies finding a correlation between F1 expression, Fn-binding, and epithelial cell adhesion, internalization, and evasion of phagocytosis $(41,47,58,72,83,105,106,116-118)$. F1 is one of seven GAS proteins possessing Fn-binding repeats that interact with the $\mathrm{N}$ terminus of Fn, which includes type I repeats 1-5, the heparin binding site. F1 also contains a region, located upstream of Fn-binding repeats, interacting with type I repeats 8 and 9, constituting the gelatin/collagen binding site. The Fnbinding repeats are found in Fn-binding proteins of many other bacteria, including proteins FnbpA and B of Staphylococcus aureus. Upon binding to Fn, they form a "tandem $\beta$-zipper which facilitates bacterial internalization via Fn-bridging (130). It should be noted that these Fn-binding proteins recognize and bind the $\mathrm{N}$-terminal region of the molecule, which is common in both the plasma $(\mathrm{pFn})$ and cellular (cFn) forms of Fn. In this work, I will describe and characterize a novel selective cFn-binding mechanism, which is unique to the Scl1 protein. 
Final Remarks. Over the last century, enormous strides have been taken to understand the pathogenesis of GAS. As detailed in the previous sections, GAS is a dynamic, highly adaptable, and often injurious human pathogen maintaining numerous virulence factors allowing for host immune evasion, tissue colonization, and disease progression. Efforts continue to unravel pathogenesis mechanisms involving GAS surface adhesins. One such adhesin is the streptococcal collagen-like protein-1 (Scl1) which over the last decade has been characterized as an important pathogenicity factor interacting with human plasma components and eukaryotic cell surface receptors. The following sections within this Chapter provide a synopsis of the Scl proteins with the subsequent Chapters devoted to the recent findings further characterizing Scl1 as a multi-functional GAS adhesin. 


\section{Table 1. Group A Streptococcus Fibronectin Binging Proteins}

\begin{tabular}{|c|c|c|c|}
\hline Fn-Binding Protein & $\begin{array}{l}\text { Fn-Binding } \\
\text { Motif }\end{array}$ & Binding Function & References \\
\hline F1 (PrtF1)/Sfb1 & HBD, GBD & $\begin{array}{l}\text { Adhesion, } \\
\text { internalization, evasion } \\
\text { of phagocytosis }\end{array}$ & $\begin{array}{l}\text { Talay et al, 1992, 1994, 2000; } \\
\text { Ozeri et al 1996, 1998; } \\
\text { Hanski and Caparon, 1992; } \\
\text { Molinari et al 1997; } \\
\text { Kreikemeyer et al, 2004; } \\
\text { Schwartz-Linck et al 2004, } \\
\text { 2006; Hyland et al, } 2007\end{array}$ \\
\hline F2 (PrtF2)/PFBP & HBD & Adhesion & $\begin{array}{l}\text { Jaffe et al, 1996; Rocha and } \\
\text { Fischetti, 1999; Kreikemeyer } \\
\text { et al, } 2004\end{array}$ \\
\hline FbaA & $?$ & Adhesion & $\begin{array}{l}\text { Terao et al, } 2001 \text { Kawabata } \\
\text { et al, } 2001\end{array}$ \\
\hline FbaB & $?$ & $\begin{array}{l}\text { Adhesion, } \\
\text { internalization }\end{array}$ & $\begin{array}{l}\text { Terao et al, 2002; Amelung et } \\
\text { al, } 2011\end{array}$ \\
\hline SOF/Sfbll & HBD & Adhesion & $\begin{array}{l}\text { Rakonjac et al, } 1995 \text {; } \\
\text { Courtney et al, 1999; Jeng et } \\
\text { al, 2003; Oehmcke et al, } \\
2004\end{array}$ \\
\hline FBP54 & HBD & Adhesion & Courtney et al, 1994, 1996 \\
\hline M protein & HBD & $\begin{array}{l}\text { Adhesion, } \\
\text { internalization, evasion } \\
\text { of phagocytosis }\end{array}$ & $\begin{array}{l}\text { Cue et al, 1998, 2001; } \\
\text { Molinari and Chhatwal, } 1999\end{array}$ \\
\hline $\begin{array}{l}\text { GADPH (glyceraldehydes- } \\
\text { 3-phosphate dehydrogenase }\end{array}$ & $?$ & Adhesion & Pancholi and Fischetti, 1992 \\
\hline Protein $\mathrm{H}$ & Fn type III & Adhesion & Frick et al, 1995 \\
\hline SfbX & HBD & adhesion & Jeng et al, 2003 \\
\hline SHR & $?$ & Adhesion & Fisher et al, 2008 \\
\hline Scl1 & $\begin{array}{l}\text { EDA/cFn type } \\
\text { III }\end{array}$ & $\begin{array}{l}\text { Adhesion, } \\
\text { internalization }\end{array}$ & $\begin{array}{l}\text { Caswell et al, 2010; Oliver- } \\
\text { Kozup et al, } 2012\end{array}$ \\
\hline
\end{tabular}

HBD: heparin-binding domain Fn type I (1-5 repeats); GBD: gelatin/collagen binding domain Fn type I (6-9 repeats) 
Streptococcal Collagen-like Proteins. Collagens comprise a ubiquitous and indispensible family of proteins that are the primary constituents contributing to the assembly and stabilization of the extracellular matrix of animals. The structural hallmark of collagens is its triple helical nature derived from three lefthanded, polyproline II-type chains forming a right-handed superhelix $(71,94)$. With at least twenty-eight types of mammalian collagen identified, its importance in cellular development and the capacity for binding to multiple matrix components, it is no surprise that pathogenic bacteria have developed surface proteins to bind collagen or structurally and functionally mimic collagen (58). Spanning now more than a decade, the identification of collagen-like sequences in prokaryotes and bacteriophages has expanded (97) and characterization of the structural stabilization and biological function has continued with much information gained by studies with the streptococcal collagen-like (Scl) proteins of GAS $(40,128)$.

Scl Genes and Protein Expression. GAS expresses two cell surfaceanchored proteins encoded by the $s c / 1$ and $s c / 2$ genes, also referred to as $s c / A$ and $s c / B$, respectively $(63,96,126)$. The $s c / 1$ and $s c / 2$ genes are located on opposite sites of the GAS chromosome and their expression is controlled by different mechanisms. The expression of $s c / 1$ is regulated at the level of transcription by the positive multiple gene regulator, Mga, similarly to several known GAS virulence factors within the Mga regulon. In contrast, control of expression of the Scl2 resides at the level of translation and depends on the number of CAAAA pentanucleotide repeats found downstream to a GTG start 
codon. Based on the number of these repeats, the scl2-coding sequence may be in-frame, resulting in expression of the full-length protein, or out-of-frame, leading to early translation termination $(64,95)$.

Scl Protein Structure. The Scl1 and Scl2 proteins are structurally similar and contain an amino-terminal signal sequence (SS) commonly found in extracellular proteins (Figure 1). The cell wall-membrane associated region (WM) contains a conserved cell-wall anchor LPXTG motif. A linker (L) region, only found in Scl1, connects the WM with the collagen-like $(C L)$ region and is composed of a series of conserved direct PGEKAPEKS repeats. The CL region of the Scl protein extends from the cell surface and is composed of a series of GXY repeats, where $G$ is glycine and $X$ and $Y$ are other amino acids. The $C L$ regions vary in length among Scl variants due to the different numbers of GXY repeats and form stable triple helices under physiological conditions. The amino terminal portion of the mature Scl proteins, designated the variable (V) region, also varies in length and primary sequence among Scl variants. Secondary structure predictions support that the $\mathrm{V}$ region is composed of two alpha-helices and may be amphipathic in nature with a probability towards forming a coiled-coil structure. As shown by electron microscopy of rotary-shadowed Scl proteins, a "Iolli-pop"-like structural organization of the homotrimeric Scl molecules is formed with the $\mathrm{CL}$ region making up a stalk of varying lengths depending on the variant and the $\mathrm{V}$ region forming a globular head $(40,128)$. 

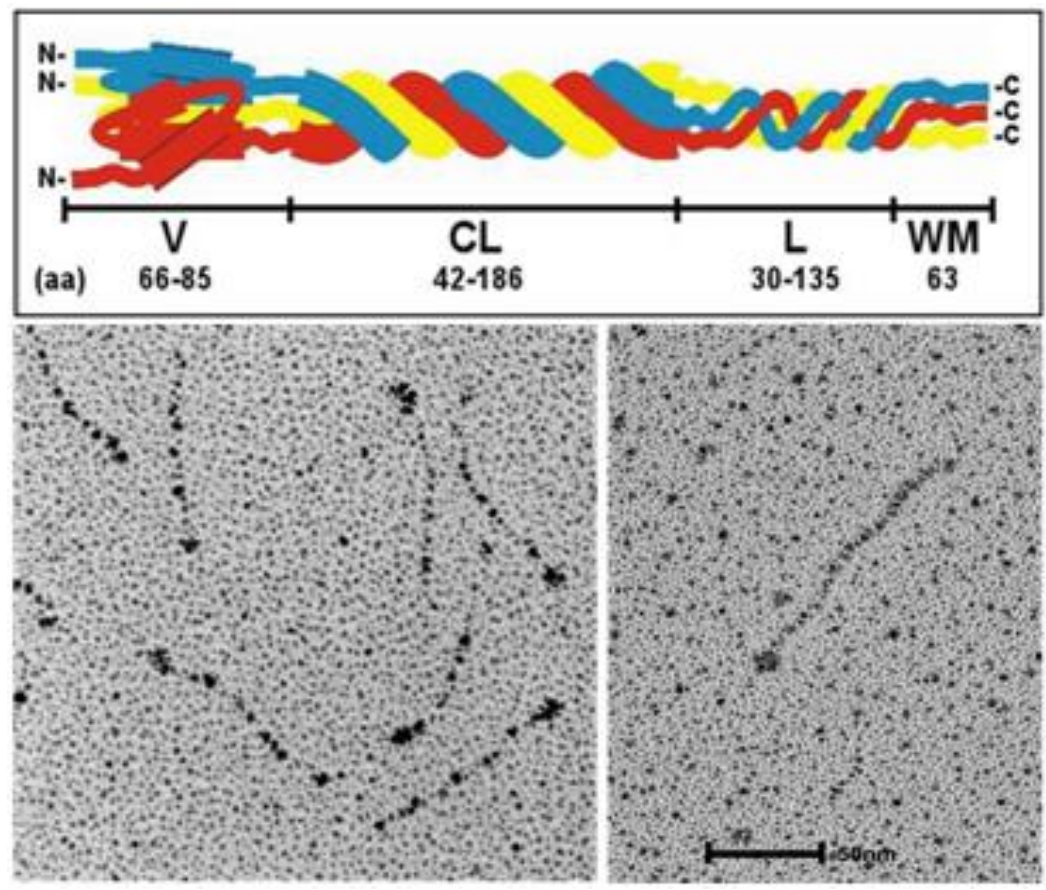

Figure 1. Structure and domain organization of Scl1. Top: Cartoon model of the Scl1 protein (not to scale). Three polypeptide chains are shown in blue, red, and yellow. Four arbitrarily designated regions and the range of amino-acid (aa) length are shown: $\mathrm{V}$, variable region; $\mathrm{CL}$, collagen-like region; $\mathrm{L}$, linker region, and WM, cell wall-membrane associated region . Bottom: Electron microscopy of rotary shadowed recombinant Scl1 proteins shows a "Iolli-pop"-like structural organization with the $\mathrm{V}$ region forming a globular head and the $\mathrm{CL}$ region forming the stalk. Scale bar represents 50 nanometers. Images within this figure are used with permission by Slawomir Lukomski. 
Stabilization of Scl Proteins. Stabilization of mammalian collagens results from the unique high content of hydroxyproline residues. Posttranslational modification by prolyl hydroxylase of proline residues constituting the $Y$ position within the GXY sequence serves as a stabilizing measure to preserve the triple helical confirmation $(8,9)$. Most information known about the triple-helix stabilization of prokaryotic collagen-like proteins is from the characterization of the Scl proteins of S. pyogenes (40) (71); however, multiple other prokaryotes such as Bacillus cereus (122), Bacillus anthracis (115), Pneumococcus (88), Clostridium (97, 127, 134), Streptococcus equi (51), and many others possess similarly structured surface proteins. Due to the lack of prolyl hydroxylase, prokaryotes are restricted to alternative mechanisms to stabilize the triple helix of the CL region (127). In the first assessment of Scl proteins for the formation of a stable triple-helical $\mathrm{CL}$ domain, $\mathrm{Xu}$ and co-workers generated recombinant Scl1 and Scl2 proteins from M1- and M28-type GAS strains, respectively. Trypsin digestion was performed to test the susceptibility of the recombinant Scl proteins to proteolytic digestion. Similar to mammalian collagens, they were relatively resistant to proteolysis suggesting the presence of a stable, collagen-like structure. Furthermore, circular dichroism spectroscopy showed a molar ellipticity maximum $(\Theta)$ for these recombinant proteins around $220 \mathrm{~nm}$, which is a characteristic of a collagen triple-helix structure. Thermal stability studies revealed a mid-melting temperature $\left(T_{m}\right)$ range for these proteins between 32-39 ${ }^{\circ} \mathrm{C}$ with an increased $\mathrm{T}_{\mathrm{m}}$ for proteins containing longer $\mathrm{CL}$ domains (128). Using knowledge gained from previous biophysical experiments 
and crystallographic models where stable collagen-like triple helices were formed with substitutions of certain amino acids in the proline and hydroxyproline positions, the authors proposed mechanisms that may participate in prokaryotic triple-helix stabilization. Such mechanisms include (i) the high content of charged amino acids with extended side chains, such as lysine and arginine that may directly interact with adjacent polypeptides; (ii) the presence of GXY triplets such as GPR, GER, GPA, GDR, GKD, and GEK previously been shown to stabilize the triple-helical structure; and importantly, (iii) the requirement of the $\mathrm{V}$ region for the trimerization and proper folding of the $C L$ region $(40,128,132,133)$.

Function of the Scl Proteins. Since no known domains could be predicted for group A streptococcal proteins Scl1 and Scl2 with bioinformatic tools, their ligands were identified using experimental approaches. Thus, different Scl variants bind human extracellular matrix proteins cellular fibronectin and laminin, collagen integrin receptors $\alpha_{2} \beta_{1}$ and $\alpha_{11} \beta_{1}$, and plasma components including the low-density lipoprotein, thrombin-activatable fibrinolysis inhibitor, complement regulatory protein factor $\mathrm{H}$ and factor $\mathrm{H}$ related protein-1. Altogether, Scl proteins are structurally related and are involved in adhesion, host-cell entry, and immune evasion. The roles of the $\mathrm{V}$ and $\mathrm{CL}$ regions of Scl1 in ligand binding, as shown in Figure 2, will be discussed separately in the following paragraphs. 


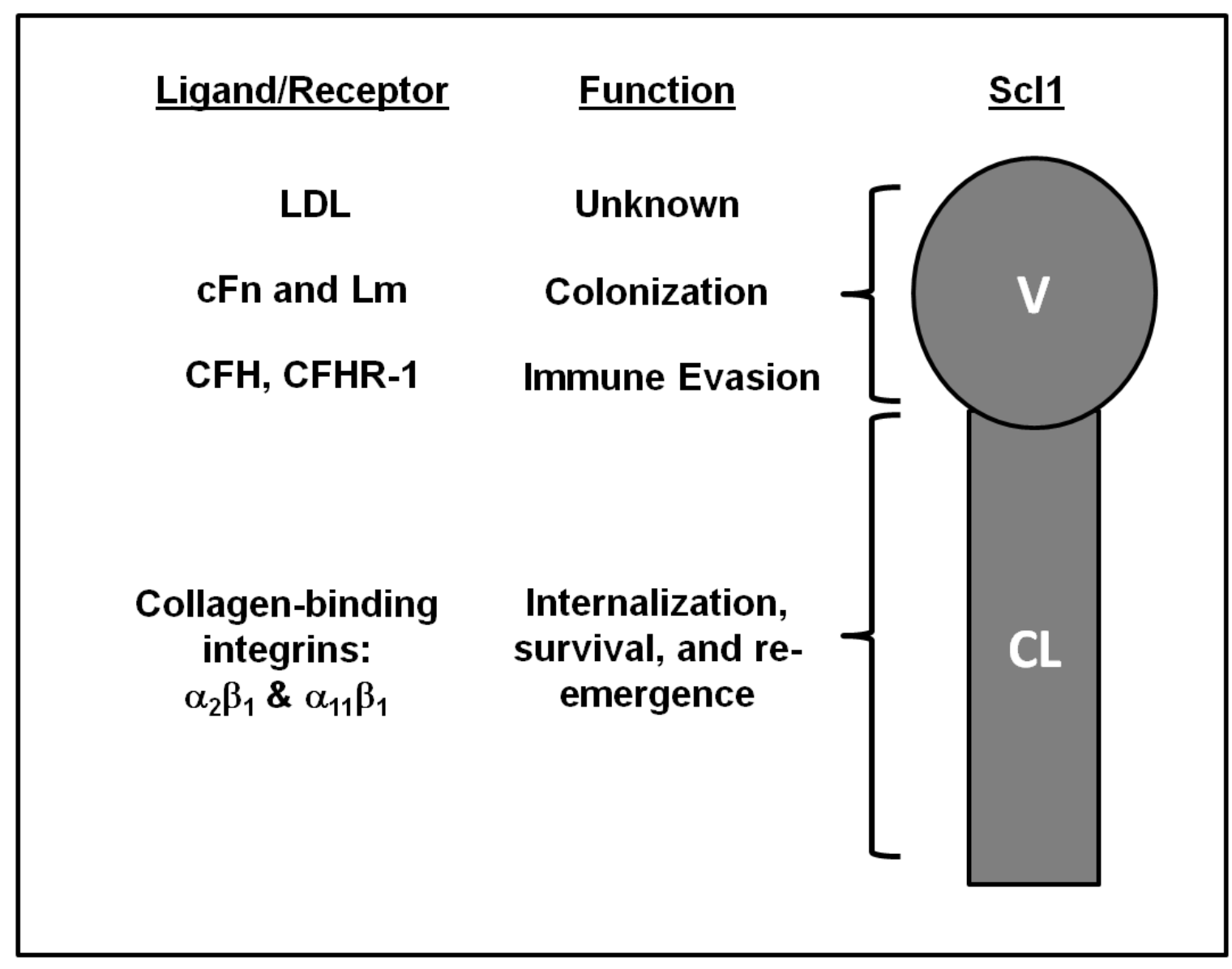

Figure 2. Dichotomous binding of the Scl1 protein to human ligands. Cartoon depiction of the variable $(\mathrm{V})$ region and collagen-like $(\mathrm{CL})$ region of the Scl1 protein. The ligand/receptor and function (if known) are shown at left for each respective region. 
Function of the CL Region of Scl1. Mammalian collagens promote essential eukaryotic cell functions, including cell attachment and migration via interactions with specific cellular receptors, the collagen-binding integrins $\alpha_{1} \beta_{1}$, $\alpha_{2} \beta_{1}, \alpha_{10} \beta_{1}$ and $\alpha_{11} \beta_{1}(46,55,62)$. Since previous research identified the structural mimicry of the Scl-CL region with mammalian collagen, it was hypothesized that Scl proteins may also act as the collagen functional mimics and interact with human collagen integrin receptors. Humtsoe et al demonstrated that recombinant Scl1.41 (rScl1.41) protein, derived from M41-type GAS, designated P176, supported the attachment of human lung fibroblasts and promoted cell spreading. This, in turn, induced intracellular signaling resulting in the phosphorylation of several molecules of the integrin pathway, including FAK, CAS, paxillin, and JNK (46). Subsequent experiments determined that the Scl-CL region was responsible for binding to $\alpha_{2} \beta_{1}$ and $\alpha_{11} \beta_{1}$, but not $\alpha_{1} \beta_{1}$ integrins. An additional study determined that the native Scl1.41 protein expressed on the GAS surface promoted GAS internalization by human pharyngeal epithelial cells, which was mediated by the Scl1 binding to $\alpha_{2} \beta_{1}$ integrin. Internalization and intracellular survival resulted in enhanced re-emergence of GAS from the human cells, thus, providing a potential mechanism to avoid antibiotic killing (11). Subsequent work established that multiple human cell types expressing $\alpha_{2} \beta_{1}$ and $\alpha_{11} \beta_{1}$ integrins internalize GAS through direct interactions between Scl1 and integrin receptors (12). These investigations were further supported by the work of Chen and coworkers, which showed a decrease in epithelial cell binding by the Scl1-negative mutants and an increased binding of the E. coli cells expressing 
Scl1 on the surface (14). In total, this work demonstrates that Scl1 is both the structural and functional collagen mimic.

Function of the $\mathbf{V}$ Region of Scl1. Extensive studies done to identify potential ligands for the Scl proteins have uncovered two distinct binding patterns associated with the $\mathrm{V}$ regions of different Scl1 variants (Figure 2). As discussed in the paragraphs below, our lab has previously identified that multiple rScl1 variants derived from GAS strains of various M-types bound to apolipoprotein B100 (ApoB100), which is a sole protein component of LDL, while others bound the complement factor $\mathrm{H}(\mathrm{CFH})$ and complement factor $\mathrm{H}$-related protein-1 (CFHR1) $(13,39,99)$. In this work, we established that those binding to LDL also interact with the extracellular matrix components, cellular fibronectin (discussed in the following Chapters), as well as, laminin. This dichotomous binding pattern is represented in Figure 2.

In Han et al, the interaction of the Scl1-ApoB100 was characterized (39). In a series of ELISA-based studies, it was identified that rScl1 proteins derived from M1-, M12-, M28-, and M41-type GAS strains bound to purified LDL/ApoB100, the primary transport molecule in lipid metabolism. Surface plasmon resonance was used to quantify the specificity of Scl1-LDL binding. To determine the binding location of LDL on the Scl1 protein, chimeric recombinant proteins, generated by domain swapping, were used in binding studies. Results showed that the $\mathrm{V}$ region supported the interaction, which was further confirmed visually with electron microscopy (EM). Rotary shadowed samples containing mixtures of rScl1.41 and LDL vesicles revealed that LDL was interacting with 
Scl1 via its globular, amino-terminal domain, indicating the $\mathrm{V}$ region. Lastly, Scl1mediated binding of LDL to GAS cells was assessed by comparing the absorption of plasma LDL and FITC-LDL to M28 wild-type and sc/1-inactivated isogenic mutant cells. Both western blot analysis and fluorescent microscopy demonstrated that only the wild-type strain expressing the native Scl1 bound LDL, whereas binding by the mutant strain was absent. The function of the Scl1LDL interaction remains unclear. However, due to that LDL receptors are found on multiple cells and are important in host defense, it was proposed that the Scl1-LDL interaction may facilitate GAS innate immune evasion by altering early inflammatory responses initiated by macrophages during GAS infection (39).

In Caswell et al, the interactions of the Scl1 proteins with CFH and CFHR1 were identified and characterized (13, 99). Affinity chromatography and subsequent mass spectrometry analysis revealed that Scl1.6 protein derived from the M6-type GAS strain bound to human plasma CFH and CFHR-1. CFH functions as a regulatory protein in the alternative complement pathway and prevents non-specific complement activation. Specifically, CFH acts as a cofactor to the serum protease, Factor I, in the cleavage of C3b and the decay of the C3convertase. This, in turn, helps to inhibit the production of opsonins and chemoattractants that would otherwise build up on self-cell surfaces and initiate phagocytic destruction. The sequestering of $\mathrm{CFH}$ on the bacterial surface mimics this mechanism and allows them to evade the innate immune response. $\mathrm{CFH}$ is composed of twenty (1-20) short consensus repeats (SCR). CFHR-1 contains 5 SCRs and SCRs 3-5 are nearly identical in sequence with the C-terminal SCRs 
18-20 of CFH. To determine the Scl1 domain supporting CFH/CFHR-1 binding, chimeric $\mathrm{rScl}$ proteins were generated through domain swapping of the $\mathrm{V}$ and $\mathrm{CL}$ regions. ELISA-based binding studies and western blot analysis showed that the Scl1-CFH/CFHR-1 interaction was mediated through the $V$ region of Scl1.6. A modified in vitro assay was performed to demonstrate that the Scl1-bound CFH indeed maintained its biological activity as a co-factor for factor Fl-mediated C3b cleavage. Finally, to assess whether native Scl1 on GAS surface supports CFH binding, a ligand affinity assay was performed using GAS cell wall material. It was shown that CFH bound a single protein band corresponding to the Scl1.6 protein. Surprisingly, this assay did not show CFH binding by the M6 protein, which was originally reported to interact with both $\mathrm{CFH}$ and complement factor $\mathrm{H}$ like protein-1 $(6,53,86)$. In total, this work confirmed CFH and CFHR-1 as ligands for the Scl1 protein of M6-type GAS and suggests that GAS may utilize the Scl1-CFH interaction to evade innate immune defenses $(13,45,49,85)$.

Final Remarks. As GAS continues to be a major human pathogen and maintains its prevalence globally, the requirement for knowledge of pathogenesis mechanisms and contributing surface-associated and secreted virulence factors remains constant. In support of this, a major finding within the present body of work as defined in the following Chapters, is that the ubiquitous, surfaceassociated Scl1 protein representing pathogenically different M-types is now among the list of GAS Fn-binding virulence factors with a unique specificity toward a spliced-variant of $\mathrm{cFn}$. This finding represents a novel and purposeful mechanism by which GAS utilizes Scl1 to secure adherence in the wounded 
tissue promoting colonization and biofilm formation, by specifically targeting the extracellular matrix during the process of wound healing. 


\section{Chapter 2:}

Streptococcal collagen-like protein-1 (Scl1) Interacts with Selected Extracellular Matrix Components 
Scl1, the multifunctional adhesin of group A Streptococcus selectively binds cellular fibronectin and laminin, and mediates pathogen internalization by human cells.

Clayton C. Caswell ${ }^{1 \ddagger}$, Heaven Oliver-Kozup ${ }^{1 \ddagger}$, Runlin Han ${ }^{1}$, Ewa Lukomska ${ }^{1}$, \& Slawomir Lukomski ${ }^{1,2}$

${ }^{1}$ Department of Microbiology, Immunology, and Cell Biology; and ${ }^{2}$ Mary Babb Randolph Cancer Center, West Virginia University School of Medicine, Morgantown, WV, USA

Published in FEMS Microbiol Let (2010) 303, 61-68 


\section{Abstract}

The streptococcal collagen-like protein-1, Scl1, is widely expressed by the wellrecognized human pathogen group A Streptococcus (GAS). Screening of human ligands for binding to recombinant Scl1 identified cellular fibronectin and laminin as binding partners. Both ligands interacted with the globular domain of Scl1, which is also able to bind the low-density lipoprotein. Native Scl1 mediated GAS adherence to ligand-coated glass cover slips and promoted GAS internalization into HEp-2 cells. This work identifies new ligands of the Scl1 protein that are known to be important in GAS pathogenesis and suggests a novel ligandswitching mechanism between blood and tissue environments, thereby facilitating host colonization and GAS dissemination. 


\section{Introduction}

Group A streptococci (GAS) typically colonize the human throat and skin causing superficial infections, such as pharyngitis and impetigo respectively. However, GAS infections may also lead to invasive diseases including necrotizing fasciitis and streptococcal toxic shock syndrome or may result in the postinfectious autoimmune sequelae acute rheumatic fever and acute glomerulonephritis (10). Host colonization is accomplished through interactions between GAS cell-surface adhesins and host cellular receptors or extracellular matrix components (ECM). Depending on the strain, GAS may express multiple surface proteins, including the streptococcal collagen-like proteins Scl1 $(21,29)$ and Scl2 $(22,28,34)$.

Structurally, Scl1 and Scl2 proteins contain a signature central collagenlike $(\mathrm{CL})$ region, which is composed of a repeating Gly-Xaa-Yaa sequence capable of adopting a stable triple helical structure similar to mammalian collagens (35). This structural mimicry by the $C L$ region has been shown to support direct interactions with collagen-binding integrins (17) and mediate GAS adherence to and internalization by human cells (5).

The amino-terminal part of the Scl proteins, termed the variable $(\mathrm{V})$ region, forms a globular domain which is protruded away from the GAS-cell surface by the $\mathrm{CL}$ region (35). The V-region sequences vary significantly between Scl1 and Scl2. In addition, the V-region sequence of each Scl protein is conserved in strains of the same M-type but differs considerably among Scls from strains of different M-types. Despite the observed sequence variation, two main ligands 
have been identified that bind different Scl1 variants via their V-regions. The Scl1.6 and Scl1.55 proteins of M6- and M55-type GAS respectively, bind human plasma glycoproteins factor $\mathrm{H}$ and the factor $\mathrm{H}$-related protein 1 (4). On the contrary, several other Scl1 variants bind the low-density lipoprotein (LDL) including Scl1 proteins of the M1-, M2-, M12-, M28-, and M41-type GAS (14). The latter Scl1.41 protein also binds integrins $\alpha_{2} \beta_{1}$ and $\alpha_{11} \beta_{1}$ via direct interaction with the $C L$ region (6). This suggests specialization in ligand binding among Scl1 proteins and their importance as pathogenicity traits.

The binding of the extracellular matrix (ECM) components by pathogens is known to be a common strategy used to establish host colonization. Several GAS cell-surface molecules have been reported to initiate this interaction including several M proteins, F1/Sfbl, F2, SOF, Sfbll, Lbp and Shr $(7,13,16,18$, $19,24,31)$. Thus, in this work, we hypothesized that Scl proteins possess binding capacities to ECM components that, in turn, would facilitate bacterial adhesion to human extracellular matrix and internalization by host cells. It is known that Scl1 is expressed by virtually all GAS strains $(21,29)$; therefore, this work further supports the role of Scl1 protein as an important accessory, multifunctional surface adhesin of GAS. 


\section{Materials and methods}

GAS strains and growth conditions. The M41-type MGAS 6183 wild-type and the $s c / 1$ mutant strain were used. The isogenic scl1 mutant of MGAS 6183 was constructed by allelic replacement as described previously (5). To prepare GAS cells for experiments, cultures were grown overnight on brain-heart infusion agar (BD Biosciences, Sparks, MD) at $37^{\circ} \mathrm{C}$ in an atmosphere of $5 \% \mathrm{CO}_{2}-20 \% \mathrm{O}_{2}$. Overnight cultures were used to inoculate Todd-Hewitt broth (BD Biosciences) supplemented with $0.2 \%$ yeast extract and the cultures were incubated at $37^{\circ} \mathrm{C}$ until they reached logarithmic phase of growth $\left(\mathrm{OD}_{600} \sim 0.5\right)$. The bacteria were collected by centrifugation, washed and then suspended in phosphate-buffered saline (PBS) at the desired cell concentration, which was verified by plating on tryptic soy agar containing 5\% sheep's blood (Remel, Lenexa, KS).

Enzyme-linked immunosorbent assays (ELISA). Recombinant Scl (rScl) proteins used in ELISA were expressed in E. coli and purified by affinity chromatography using the Strep-tag II system (IBA-GmbH, Goettingen, Germany) as described previously $(15,35)$. Briefly, the DNA fragments of several scl1 and scl2 alleles, encoding the extracellular portions of the Scl1 and Scl2 proteins, were amplified by polymerase chain reaction with Deep Vent Taq Polymerase (New England Biolabs, Beverly, MA) and cloned into the pASK-IBA2 vector designed for periplasmic expression.

Recombinant Scl proteins $(0.5 \mu \mathrm{M})$ were immobilized onto Strep-Tactincoated microplate wells for 1.5 hours at room temperature. Following overnight 
blocking with Tris-buffered saline (TBS) supplemented with $1 \%$ bovine serum albumin (BSA) at $4^{\circ} \mathrm{C}, 1 \mu \mathrm{g}$ of each ligand that included plasma fibronectin (pFn), cellular fibronectin (cFn), laminin (Lm), bovine collagen types I and IV, decorin, heparin, and fibrinogen (all proteins were purchased from Sigma) was added to triplicate wells and the mixture was incubated at room temperature for 1 hour. rScl-bound ligands were detected with specific primary antibodies and appropriate secondary antibodies conjugated to horseradish peroxidase (HRP). The HRP reaction was developed with 2, 2'-azino-bis (3-ethylbenzthiazoline-6sulfonic acid) (ABTS) substrate and recorded at $\mathrm{OD}_{415}$ after 15 minutes of color development.

In the ligand competition experiments, purified cFn and Lm were used in a molar ratio 1:1. First, the primary ligands e.g., cFn or Lm, were added to triplicate wells immobilized with $\mathrm{P} 176$ and incubated for $1 \mathrm{~h}$ at room temperature. Following washes with TBS, secondary ligands were added to the appropriate wells e.g., Lm was added to wells containing Scl1-cFn complex and vice versa; samples were incubated for $1 \mathrm{~h}$ at room temperature. Subsequently, the ELISA proceeded as described above.

GAS whole-cell attachment assay to ECM proteins. To generate GFPexpressing GAS cells, the wild-type strain, the sc/1-inactivated mutant, and mutant complemented in-trans for Scl1.41-protein expression (plasmid pSL230) (5) were transformed with the plasmid pSB027 (8). Glass cover slips were placed in the wells of 24-well tissue culture plates and coated with $2.5 \mu \mathrm{g}$ of purified 
ECM proteins or BSA overnight at $4^{\circ} \mathrm{C}$, and subsequently blocked with $1 \% \mathrm{BSA}$ in TBS for $1 \mathrm{~h}$. Approximately $1 \times 10^{7} \mathrm{CFU}$ of fluorescent GAS cells were added to each well for $1 \mathrm{~h}$ at room temperature and unbound cells were removed by washing with PBS. ECM-bound GAS cells were fixed with 3\% para-formaldehyde in PBS for $30 \mathrm{~min}$. The cover slips were removed from the wells, air-dried, placed on microscope slides, and viewed by fluorescent microscopy using a 450-490 nm excitation channel at $400 x$ and $1000 x$ magnification. For quantification, GAS cells were counted in 10 random fields under 1000x magnification.

GAS internalization assays. An antibiotic protection assay was employed for GAS internalization as described previously (5) with some modifications. Briefly, human HEp-2 cells were grown in 24-well tissue culture plates until semiconfluent. All co-culture experiments were performed in serum-free and ECMfree Delbeco's Modified Eagle Medium (DMEM). For ECM treatment, $10 \mathrm{ml}$ of $1 \times 10^{7} \mathrm{CFU} / \mathrm{ml}$ of each prepared GAS strain was pre-incubated with $15 \mu \mathrm{g}$ of purified cFn or Lm for 1 hour at room temperature on an end-over-end rotator. Subsequently, $\sim 1 \times 10^{6} \mathrm{CFU}$ of ECM-treated or ECM-untreated wild type or scl1inactivated mutant GAS were co-cultured with the HEp-2 cells (MOI 1:100) for 2 hours at $37^{\circ} \mathrm{C}$. Cell layers were washed with PBS, and culture medium containing $100 \mu \mathrm{g} \cdot \mathrm{ml}^{-1}$ gentamicin and $5 \mu \mathrm{g} \cdot \mathrm{ml}^{-1}$ penicillin $\mathrm{G}$ was added to each well to kill extracellular bacteria. After 2 hours, the medium was removed and the cells were washed with PBS. To determine the level of GAS internalization, the epithelial cells were lysed in distilled water and serial dilutions were plated onto 
blood agar. The internalization level of ECM-untreated wild-type strain was considered $100 \%$.

Statistical analysis. Statistical significance was determined using a two-tailed paired Student's $t$-test. The results were considered statistically significant with $P<0.05\left(^{*}\right), P<0.01\left(^{* *}\right)$, and $P<0.001\left(^{* * *}\right)$.

\section{Results and discussion}

M41-serotype strains of GAS have emerged as a major cause of streptococcal impetigo during 1950s and 1960s (2). They were isolated from skin infections in several geographical locations, including Minnesota (32), Alabama (12), and Trinidad (11) with frequencies of $12-14 \%$ of all cases. The M41-type isolates were also reported in a recent GAS surveillance study of patients with invasive infections in the United States (25). Strain MGAS 6183 used here was cultured from a leg abscess during the epidemics of invasive GAS infections in Texas.

We have previously reported that the recombinant Scl1.41 protein, designated P176, bound human collagen receptors via its collagen-like (CL) region and $L D L$ via the variable $(V)$ region $(6,14)$. Here, we evaluated the binding of an array of potential human ligands, including several ECM proteins, to the recombinant P176 by ELISA (Fig. 1a). We also used recombinant construct P163, derived from Scl2 protein of M28-type GAS, for which no ligands have been identified to date. None of the ligands tested here bound to the recombinant protein $\mathrm{P} 163$. No significant binding to P176 was detected for fibrinogen, decorin, 
heparin, and collagens I and IV (data not shown). Remarkably, P176 bound cellular fibronectin (cFn) but not plasma fibronectin $(\mathrm{pFn})$. The observation that Scl1 binds to cFn but not $\mathrm{pFn}$ is novel and very intriguing. Various forms of $\mathrm{Fn}$ are products of alternatively spliced mRNA transcript of a single gene containing about 50 exons (1). The $\mathrm{pFn}$ form is predominantly produced by hepatocytes and circulates in plasma as a covalently linked dimer. More heterogeneous cFn is produced by many cell types and forms large aggregated structures known as fibronectin fibrils. The cFn comprises a large group of isoforms produced from splicing events that may or may not include the type III repeats called extra domains, EDA and EDB, lacking in pFn (26). It is currently not known whether the presence of the extra domains or supra structural organization is responsible for the selective binding of $\mathrm{cFn}$ to Scl1 protein.

In addition to cFn, P176 also bound laminin (Lm). The cFn and Lm binding to P176 was concentration-dependent indicating binding specificity (Fig. 1a, inset). The laminins comprise a family of $A, B_{1}$ and $B_{2}$ heterotrimeric glycoproteins that are constituents of basal lamina and are found in virtually all human tissues (1). Various isoforms of laminin exist that are associated with characteristic tissue distribution. Early studies by Switalski et al. described GAS binding to Lm, although the GAS product responsible for this binding was not identified (30). Terao et al. identified a GAS Lm-binding protein, designated Lbp (31), which was recently characterized as primarily a zinc-binding protein with capacity to bind Lm (20). GAS interactions with Lm were also attributed to another streptococcal protein Shr that primarily binds human plasma 
hemoproteins (13). Thus, unrelated surface proteins of GAS possess binding capacities towards extracellular matrix components Fn and Lm.

Since both cFn and Lm contain the collagen-binding domains (1), we could not exclude a possibility that the CL region of Scl1 was responsible for ECM binding. Therefore, we constructed a chimeric recombinant protein by domain swapping consisting of the V-region of P176 and the CL-region of the ECM-binding-negative protein P163. The resulting construct P181 bound cFn and $\mathrm{Lm}$ indicating that ECM-binding is mediated by the P176-V region (Fig. 1a). We next devised a competition assay to investigate whether cFn and Lm binding is localized to the same site within P176 V-region (Fig. 1b). First, immobilized P176 was incubated with one of the primary ECM ligands, cFn or Lm, and then incubated with an alternate secondary ECM ligand. Sets of triplicate wells were immunoreacted with antibodies specific for both ECM ligands to assess the presence of $\mathrm{cFn}$ and $\mathrm{Lm}$ attached to P176. Immunoreactivity of the same amounts of P176-cFn and P176-Lm were considered as 100\% binding (Fig. 1b; bars 1-2). Pre-incubation of P176 with cFn did not prevent Lm binding (bar 4) nor Lm displaced the cFn from P176 (bar 3). Likewise, pre-incubation with Lm did not prevent cFn binding to P176 (bar 5) nor cFn was able to displace the Lm from P176 (bar 6). Our data suggest that under these experimental conditions, the cFn and Lm did not compete for binding to P176.

Binding between rScl1.41 construct P176 and ECM proteins may not translate into binding between GAS-expressed Scl1.41 protein and ECM components. Therefore, a whole-cell binding assay (Fig. 2) was carried out using 
the wild-type MGAS 6183 strain, the sc/1-inactivated isogenic mutant, and the mutant complemented with plasmid pSL230 expressing in-trans the Scl1.41 protein (5). All three strains were first transformed with the plasmid pSB027 to generate GFP-expressing cells (Fig. 2a, images at left). The stability of two plasmids pSL230 and pSB027 within the complemented mutant strain was confirmed by isolating total DNA from these cells (Fig. 2d). Fluorescent GAS strains were next tested for binding to ECM-coated glass cover slips (Fig. 2a, images at middle and right columns). More fluorescent wild-type cells were seen attached to the cover slips coated with cFn and Lm, as compared with sc/1 mutant GAS. Furthermore, complementation of the scl1 mutant with pSL230 considerably increased cell binding to both ECMs. Quantitative analysis by counting the numbers of GAS cells in random fields fully supported visual observations (Fig. 2b). The sc/1-inactivated mutant bound $30 \%$ and $45 \%$ less to cFn and Lm, respectively, compared to the wild-type strain. Importantly, the complementation of mutant for Scl1.41 expression restored the wild-type levels of binding to both cFn and Lm, indicating that this phenotype was due to the lack of Scl1 expression. Residual cFn binding by the Scl1 mutant could be attributed to the presence of the prtf2 gene in this strain (5) encoding an additional Fnbinding protein, F2 (18). Similarly, the observed binding of the Scl1-deficient mutant to Lm could be attributed to Lbp and Shr expression; however, the M41type GAS was not included in the studies that characterized these ECM-binding proteins $(13,31)$. Since $I b p$ and shr genes are conserved among GAS strains of various M types, we used PCR to demonstrate the presence of both genes in 
M41-type strain MGAS 6183 (Fig. 2c). Altogether, our results demonstrate that Scl1.41 protein is an important surface adhesin that selectively binds to human cellular fibronectin and laminin and significantly contributes to ECM-GAS interactions.

GAS interactions with ECM components have been exhaustively reported in the literature and much effort has been directed toward understanding its function in GAS adherence and internalization pertaining to human disease (9). The bulk of that work focuses on Fn, although the effect of exogenous cFn on GAS internalization was not specifically investigated. Far less is known about the contribution of Lm to GAS adherence and internalization. Recently, the Lmbinding protein Lbp of the M1-type strain was shown to facilitate the adherence to and internalization by HEp-2 cells; however, the observed decrease in internalization of the Ibp mutant was not statistically significant compared with the wild-type strain (31). In addition, a mutant strain of the M49-type GAS deficient in surface protein Shr, which binds both soluble Fn and Lm, showed decreased adherence to HEp-2 cells compared with the parental strain (13).

In our current studies, the HEp-2 cells were co-cultured with the wild type or the isogenic sc/1-inactivated mutant GAS that were either treated or untreated with $\mathrm{cFn}$ or Lm. Following internalization, the numbers of surviving intracellular bacteria were determined. The Scl1-deficient GAS cells were internalized significantly less than the wild type strain in ECM-free medium (Fig. 3). Following pre-incubation with cFn and Lm, the wild type strain exhibited about a 4- and 6.5fold increase in internalization, respectively, compared to ECM-untreated cells. 
The scl1-inactivated strain pre-incubated with $\mathrm{cFn}$ and Lm also showed about 2.2- and 2.8-fold increase in internalization compared to the ECM-untreated mutant cells; however, the overall levels of mutant internalization were lower compared to wild-type strain under each corresponding experimental conditions, emphasizing the contribution of Scl1 to cell invasion by GAS. It should be noted that the in vivo relevance of GAS internalization by human cells mediated by ECM binding has been debated in recent years. In spite of this, recent investigations using NMR spectroscopy, circular dichroism analyses, and experiments with monoclonal antibodies identified structural changes exerted by fibronectin upon binding to bacterial proteins that result in an enhanced Fn recognition by integrins $(3,23)$. It is tempting to speculate that Scl1 binding to cFn and Lm may exert similar biological effects.

It was shown previously by our group that Scl1 from M41-type GAS binds the human collagen integrin receptors, which mediates GAS internalization by host cells $(5,6)$. Integrins bind directly the GLPGER sequence within the Scl1-CL region. Here, we show the $\mathrm{V}$ region of the same Scl1.41 protein binds to $\mathrm{cFn}$ and Lm, which also increases GAS internalization by HEp-2 cells. We think it is unlikely that $\mathrm{cFn}$ and $\mathrm{Lm}$ binding to the globular $\mathrm{V}$ domain affects Scl1-CL region binding to $\alpha_{2} \beta_{1}$ and $\alpha_{11} \beta_{1}$; however, we cannot fully exclude such a possibility. The HEp-2 cells express the $\alpha_{2}, \alpha_{3}, \alpha_{5}$, and $\beta_{1}$ integrin subunits (5), thus are capable of producing the $\alpha_{2} \beta_{1}, \alpha_{3} \beta_{1}$, and $\alpha_{5} \beta_{1}$ heterodimers with ability to bind collagen, laminin, and fibronectin, respectively (33). The $\alpha_{11} \beta_{1}$ integrin expression is restricted to fibroblasts (27) and, thus, may not be present on the surface of 
HEp-2 cells. Therefore, Scl1 may be contributing to internalization of M41-type GAS by HEp-2 cells by two mechanisms: direct binding to the $\alpha_{2} \beta_{1}$ integrin, and ECM-bridging mechanism via integrins $\alpha_{3} \beta_{1}$ and $\alpha_{5} \beta_{1}$.

In a final series of experiments we tested several available rScl1 and rScl2 constructs for binding to the same panel of human ligands employing an ELISAbased assay. Similar to P176, no significant binding by any rScl protein was detected for fibrinogen, decorin, heparin, collagens type I and IV (data not shown). In general, the recombinant rScl1 constructs, derived from Scl1 proteins, bound CFn and Lm (Fig. 4a); while the Scl2-protein-based constructs P163, P177, and P178 were ECM-binding negative. Furthermore, none of the rScl1 proteins tested bound $\mathrm{pFn}$, which is in agreement with our previous reports showing that those rScl1 proteins did not bind pFn from human plasma by affinity chromatography $(4,15)$. All LDL-binding constructs derived from Scl1 proteins of the M1-, M28-, M41-, M12-, M2-, and M52-type GAS (15) showed ECM binding, although to varying degrees. However, the CFH/CFHR-1-binding rScl1s originating from the M6- and M55-type GAS (4) did not show any significant binding to ECM ligands. In order to determine the region of Scl1 responsible for binding to ECM proteins, an ELISA was performed using chimeric rScl constructs generated by domain-swapping (Fig. 4b). We employed two types of chimeric molecules: (i) derived from the ECM-binding positive (+) construct P144 (Scl1.1 of M1-type GAS) and the ECM-binding negative (-) construct P177 (Scl2 of M4type GAS); and (ii) constructs derived from the ECM-binding positive P144 and the ECM-binding negative P179 (Scl1 of M6-type GAS). The rScl1 (+)-rScl2 (-) 
chimeric construct P183 (P144V/P177CL) but not P184 (P177V/P144CL) bound cFn and Lm. Likewise, the rScl1 (+)-rScl1 (-) chimeric construct P213 (P144V/P179CL) but not P212 (P179V/P144CL) bound cFn and Lm. These data strongly indicate that, indeed, the Scl1-V region is responsible for mediating interactions with ECM proteins.

The present and previous results underscore the functional diversity of the Scl1-V region. Of particular interest to us is the emergence of two main binding patterns among Scl1 variants. The more common pattern includes binding of plasma LDL and extracellular matrix components cFn and Lm, which may represent an intriguing adaptation of Scl1 to either the blood or tissue environment. Our previous molecular evolutionary genetic analysis identified an elevated constraint of the Scl1-V region sequence suggesting that this region responds to selective pressure (21). Inasmuch as the amino acid sequence in the $\mathrm{V}$ region differs between Scl1 proteins of different M-types, the prediction of two a-helices $(14,29)$ and the globular structure of the Scl1-V domain $(15,35)$ seem to be conserved among all Scl1 proteins. The present work provides a platform for future investigations that will determine the Scl1-ECM binding affinities and identify the specific amino acid sequences or structural motifs of Scl1 variants that constitute the molecular basis for the Scl1-ligand (ECM, LDL, and CFH) recognition. 


\section{Acknowledgements}

We thank S. Beres for providing plasmid pSB027. This work was supported by the National Institutes of Health Grant Al50666 and by a research grant from the West Virginia University Health Science Center, Office of Research and Graduate Education (to S.L). H. Oliver-Kozup was supported by a grant from the West Virginia Graduate Student Fellowships in Science, Technology, Engineering and Mathematics (STEM) program. 


\section{References}

1. Alberts, B., D. Bray, J. Lewis, M. Raff, K. Roberts, and J. D. Watson. 1994. Molecular biology of the cell, 3rd ed. Garland Publishing, New York, N. Y.

2. Anthony, B. F. 2000. Streptococcal pyoderma, p. 144-151. In D. L. Stevens and E. L. Kaplan (ed.), Streptococcal infections. Oxford University Press, New York, N. Y.

3. Bingham, R. J., E. Rudino-Pinera, N. A. Meenan, U. Schwarz-Linek, J. P. Turkenburg, M. Höök, E. F. Garman, and J. R. Potts. 2008. Crystal structures of fibronectin-binding sites from Staphylococcus aureus FnBPA in complex with fibronectin domains. Proc Natl Acad Sci U S A 105:12254-12258.

4. Caswell, C., R. Han, K. Hovis, P. Ciborowski, D. Keene, R. Marconi, and S. Lukomski. 2008. The Scl1 protein of M6-type group A Streptococcus binds the human complement regulatory protein, factor $\mathrm{H}$, and inhibits the alternative pathway of complement. Mol Microbiol 67:584-596.

5. Caswell, C., E. Lukomska, N. Seo, M. Höök, and S. Lukomski. 2007. Scl1dependent internalization of group A Streptococcus via direct interactions with the $\alpha_{2} \beta_{1}$ integrin enhances pathogen survival and re-emergence. Mol Microbiol 64:1319-1331.

6. Caswell, C. C., M. Barczyk, D. R. Keene, E. Lukomska, D. E. Gullberg, and S. Lukomski. 2008. Identification of the first prokaryotic collagen sequence motif that mediates binding to human collagen receptors, integrins $\alpha_{2} \beta_{1}$ and $\alpha_{11} \beta_{1}$. J Biol Chem 283:36168-36175.

7. Courtney, H. S., H. C. Chiang, J. L. Thacker, and J. B. Dale. 1999. Serum opacity factor is a major fibronectin-binding protein and a virulence determinant of M type 2 Streptococcus pyogenes. Mol Microbiol 32:89-98.

8. Cramer, T., Y. Yamanishi, B. E. Clausen, I. Forster, R. Pawlinski, N. Mackman, V. H. Haase, R. Jaenisch, M. Corr, V. Nizet, G. S. Firestein, H. P. Gerber, N. Ferrara, and R. S. Johnson. 2003. HIF-1alpha is essential for myeloid cell-mediated inflammation. Cell 112:645-657.

9. Cue, D., P. E. Dombeck, and P. P. Cleary. 2000. Intracellular invasion by Streptococcus pyogenes: invasins, host receptors, and relevance to human disease, p. 27-33. In V. A. Fischetti, R. P. Novick, J. J. Ferretti, D. A. Portnoy, and J. I. Rood (ed.), Gram-positive pathogens. ASM Press, Washington, D.C.

10. Cunningham, M. W. 2000. Pathogenesis of group A streptococcal infections. Clin Microbiol Rev 13:470-511.

11. Dillon, H. C., C. W. Derrick, and M. S. Dillon. 1974. M-Antigens common to pyoderma and acute glomerulonephritis. J Infect Dis 130:257-267.

12. Dillon, H. C., and L. W. Wannamaker. 1971. Skin infections and acute glomerulonephritis, report of a symposium. Military Medicine 136:122-127.

13. Fisher, M., Y.-S. Huang, X. Li, K. S. McIver, C. Toukoki, and Z. Eichenbaum. 2008. Shr Is a broad-spectrum surface receptor that contributes to adherence and virulence in group A Streptococcus. Infect Immun 76:5006-5015.

14. Han, R., C. C. Caswell, E. Lukomska, D. R. Keene, M. Pawlowski, J. M. Bujnicki, J. K. Kim, and S. Lukomski. 2006. Binding of the low-density 
lipoprotein by streptococcal collagen-like protein Scl1 of Streptococcus pyogenes. Mol Microbiol 61 (2): 351-67.

15. Han, R., A. Zwiefka, C. C. Caswell, Y. Xu, D. R. Keene, E. Lukomska, Z. Zhao, M. Höök, and S. Lukomski. 2006. Assessment of prokaryotic collagenlike sequences derived from streptococcal Scl1 and Scl2 proteins as a source of recombinant GXY polymers. Appl Microbiol Biot 72:109-115.

16. Hanski, E., and M. Caparon. 1992. Protein F, a fibronectin-binding protein, is an adhesin of the group A Streptococcus Streptococcus pyogenes. PNAS 89:6172-6176.

17. Humtsoe, J. O., J. K. Kim, Y. Xu, D. R. Keene, M. Höök, S. Lukomski, and K. K. Wary. 2005. A streptococcal collagen-like protein interacts with the $\alpha_{2} \beta_{1}$ integrin and induces intracellular signaling. J. Biol. Chem. 280:13848-13857.

18. Jaffe, J., S. Natanson-Yaron, M. G. Caparon, and E. Hanski. 1996. Protein F2, a novel fibronectin-binding protein from Streptococcus pyogenes, possesses two domains. Mol Microbiol 21:373-384.

19. Kreikemeyer, B., S. R. Talay, and G. S. Chhatwal. 1995. Characterization of a novel fibronectin-binding surface protein in group A streptococci. Mol Microbiol 17:137-145.

20. Linke, C., T. T. Caradoc-Davies, P. G. Young, T. Proft, and E. N. Baker. 2009. The laminin-binding protein Lbp from Streptococcus pyogenes is a zincreceptor. J Bacteriol doi:10/1128JB.00485-09.

21. Lukomski, S., K. Nakashima, I. Abdi, V. J. Cipriano, R. M. Ireland, S. D. Reid, G. G. Adams, and J. M. Musser. 2000. Identification and characterization of the $s c l$ gene encoding a group A Streptococcus extracellular protein virulence factor with similarity to human collagen. Infect Immun 68:6542-6553.

22. Lukomski, S., K. Nakashima, I. Abdi, V. J. Cipriano, B. J. Shelvin, E. A. Graviss, and J. M. Musser. 2001. Identification and characterization of a second extracellular collagen-like protein made by group A Streptococcus: control of production at the level of translation. Infect Immun 69:1729-1738.

23. Margarit, I., S. Bonacci, G. Pietrocola, S. Rindi, C. Ghezzo, M. Bombaci, V. Nardi-Dei, R. Grifantini, P. Speziale, and G. Grandi. 2009. Capturing hostpathogen interactions by protein microarrays: identification of novel streptococcal proteins binding to human fibronectin, fibrinogen, and C4BP. Faseb $\mathbf{J}$ doi:10.1096/fj.09-131458.

24. Molinari, G., and G. S. Chhatwal. 1998. Invasion and survival of Streptococcus pyogenes in eukaryotic cells correlates with the source of the clinical isolates. $\mathbf{J}$ Infect Dis 177:1600-1607.

25. O'Loughlin, R., A. Roberson, P. Cieslak, R. Lynfield, K. Gershman, A. Craig, B. Albanese, M. Farley, N. Barrett, N. Spina, B. Beall, L. Harrison, A. Reingold, C. Van Beneden, and 2007. The epidemiology of invasive group A streptococcal infection and potential vaccine implications: United States, 20002004. Clin Infect Dis 45:853-862.

26. Pankov, R., and K. M. Yamada. 2002. Fibronectin at a glance. J Cell Sci 115:3861-3863.

27. Popova, S. N., E. Lundgren-Akerlund, H. Wiig, and D. Gullberg. 2007. Physiology and pathology of collagen receptors. Acta Physiol 190:179-187. 
28. Rasmussen, M., and L. Björck. 2001. Unique regulation of SclB-a novel collagen-like surface protein of Streptococcus pyogenes. Infect Immun 40:14271438.

29. Rasmussen, M., A. Eden, and L. Björck. 2000. SclA, a novel collagen-like surface protein of Streptococcus pyogenes. Infect Immun 68:6370-6377.

30. Switalski, L. M., P. Speziale, M. Höök, T. Wadstrom, and R. Timpl. 1984. Binding of Streptococcus pyogenes to laminin. Journal of Biological Chemistry 259:3734-3738.

31. Terao, Y., S. Kawabata, E. Kunitomo, I. Nakagawa, and S. Hamada. 2002. Novel laminin-binding protein of Streptococcus pyogenes, Lbp, is involved in adhesion to epithelial cells. Infect Immun 70:993-997.

32. Top, F. H., Jr., L. W. Wannamaker, W. R. Maxted, and B. F. Anthony. 1967. $\mathrm{M}$ antigens among group A streptococci isolated from skin lesions. J Exp Med 126:667-685.

33. Watt, F. M. 2002. Role of integrins in regulating epidermal adhesion, growth, and differentiation. EMBO Journal 21:3919-3926.

34. Whatmore, A. M. 2001. Streptococcus pyogenes sclB encodes a putative hypervariable surface protein with a collagen-like repetitive structure. Microbiol 147:419-429.

35. Xu, Y., D. R. Keene, J. M. Bujnicki, M. Höök, and S. Lukomski. 2002. Streptococcal Scl1 and Scl2 proteins form collagen-like triple helices. J Biol Chem 277:27312-27318. 
Figures and Figure Legends
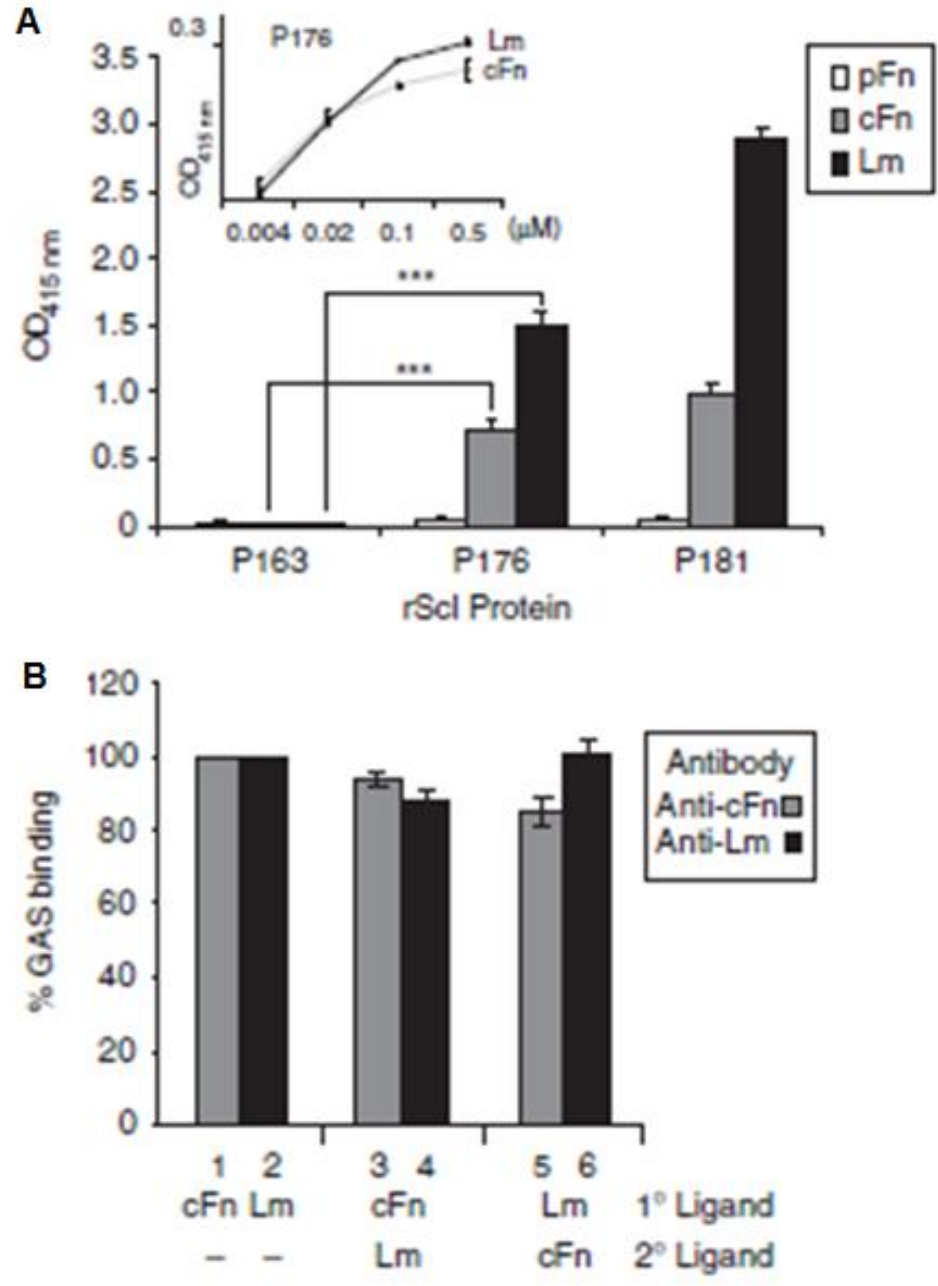

Figure 1. Characterization of ECM binding to rScl1.41 protein. (a) Recombinant Scl1.41 protein (P176) binds cellular fibronectin and laminin. Recombinant proteins P176, P163, and P181 containing portions of P176 and P163 were tested by ELISA for binding to purified plasma fibronectin $(p F n)$, cellular fibronectin (cFn), or laminin (Lm). Graph bars represent the mean $\mathrm{OD}_{415}$ $\pm S D$ of triplicate wells. (Inset): Concentration-dependent binding of cFn and Lm to P176. (b) Competition between cFN and Lm for binding to P176. Immobilized recombinant protein P176 was pre-incubated with either cFN prior to incubation with $\mathrm{Lm}$ and vice versa. Scl1-ECM complexes were immunoreacted with anti-cFn and anti-Lm Abs. Immunoreactivity of the same amounts of P176-cFn and P176$\mathrm{Lm}$ were considered as $100 \%$ binding. Data is from a single experiment that is representative of three independent experiments. 
A
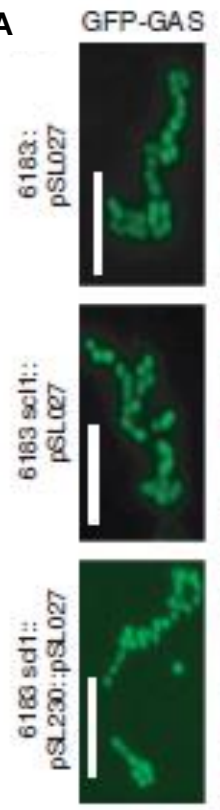

GAS-cFN
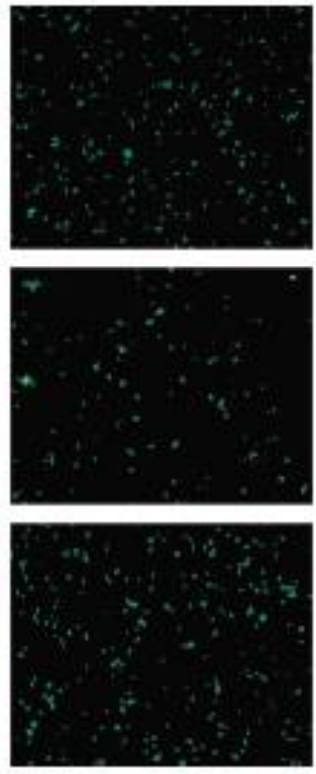

GAS-Lm
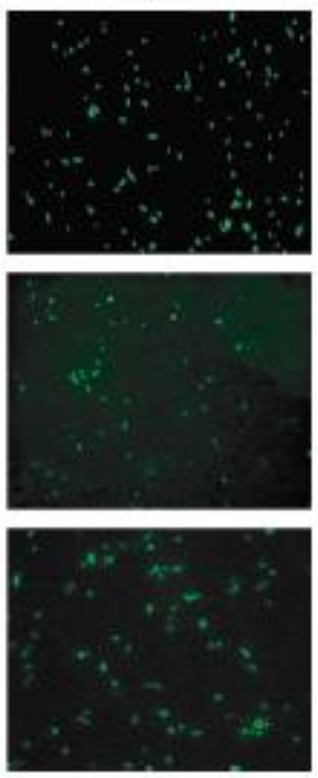

B

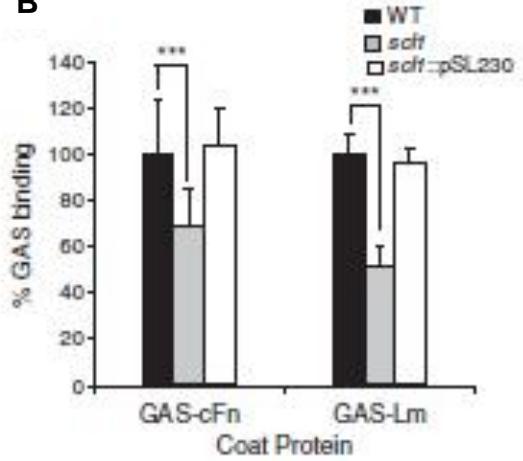

C

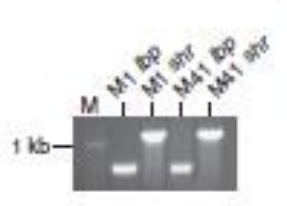

D

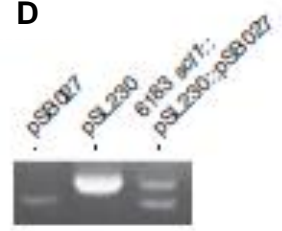

Figure 2. Scl1.41-mediated binding of GAS cells to ECM proteins. (a) Attachment of GAS cells expressing GFP marker on pSB027 to ECM-coated cover slips. Bound wild-type strain (6183::pSB027), the scl1-inactivated mutant (6183 scl1::pSB027), and the scl1 mutant complemented with plasmid pSL230 to express Scl1.41 (6183 sc/1::pSL230::pSB027) were examined under the fluorescent microscope at 400x and 1000x magnification. Scale bars represent $10 \mu \mathrm{m}$. (b) Quantification of ECM-bound GAS. GAS cells attached to ECM-coated cover slips were counted at 1000x magnification. Binding of the wild-type GAS was considered $100 \%$. Statistical significance was determined at a level of $P<$ $\left.0.001{ }^{* * *}\right)$. (c) Presence of the lbp and shr genes in M1- and M41-type GAS. Genomic DNA from MGAS 5005 (M1) and MGAS 6183 (M41) were used as templates for PCR using primer sets: IbpFor (ACCGTCTGTAAATGATGTGG) and IbpRev (CATATGATGCTTACCAAGTTG), and shrFor (GTGCGTTTGTGCAATATCTG) and shrRev (AGCGTATAGGTTCCTTCTGTG). PCR products were detected by ethidium bromide agarose gel electrophoresis. M; 1-kb DNA marker. (d) Stability of pSB027 and pSL230 in MGAS 6183. The presence of Scl1.41-expressing plasmid pSL230 and GFP-expressing plasmid pSB027 in the complemented sc/1 mutant strain was tested. GAS genomic DNA was isolated and compared with pSL230 and pSB027 plasmid markers in ethidium bromide agarose gel. 


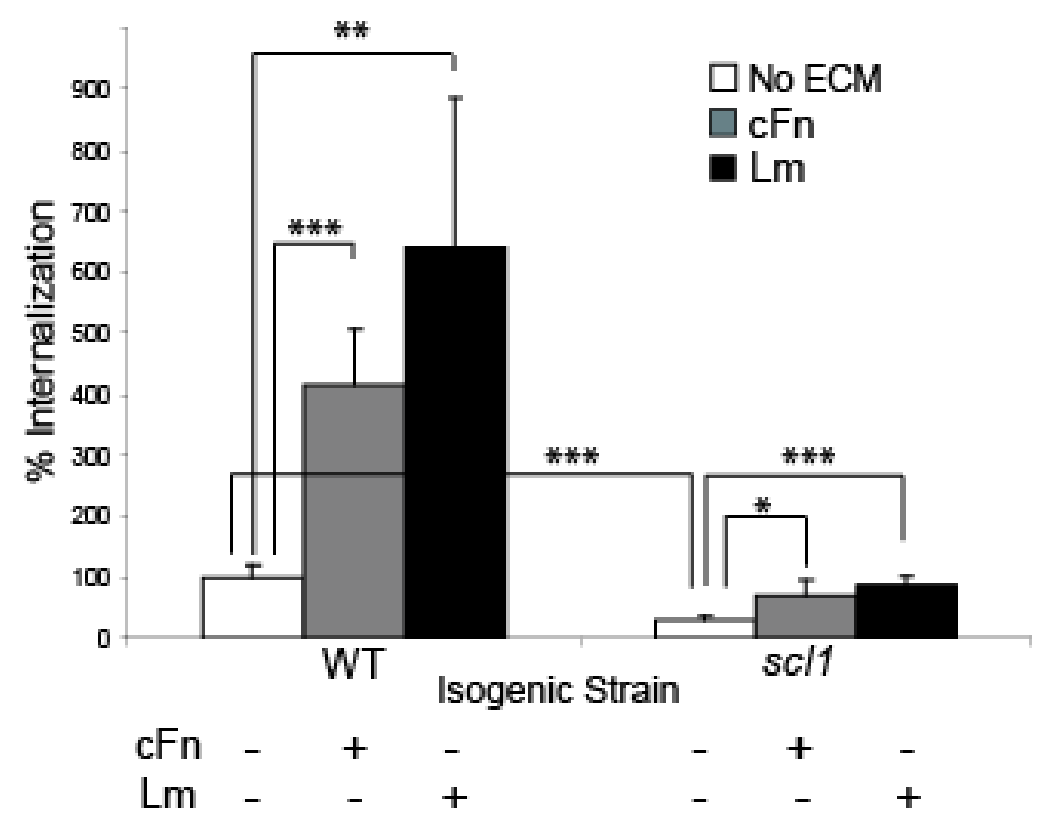

Figure 3. Scl1-ECM interactions mediate GAS internalization by human epithelial cells. HEp-2 cells were cocultured for $2 \mathrm{~h}$ in ECM-free media with the wild-type (WT) and the scl1-inactivated (scl1) GAS cells that were either retreated or untreated with $\mathrm{cFn}$ or $\mathrm{Lm}$. Following antibiotic treatment, the numbers of intracellular bacteria were determined by plating the eukaryotic-cell lysates on blood agar. The results obtained with ECM-untreated wild-type GAS were considered $100 \%$. All means (graphic bars) and standard deviations (error bars) are combined values from three independent experiments. Statistical significance was determined at a level of ${ }^{*} P \leq 0.05,{ }^{* \star} P \leq 0.01$, and ${ }^{* * *} P \leq$ 0.001 . 

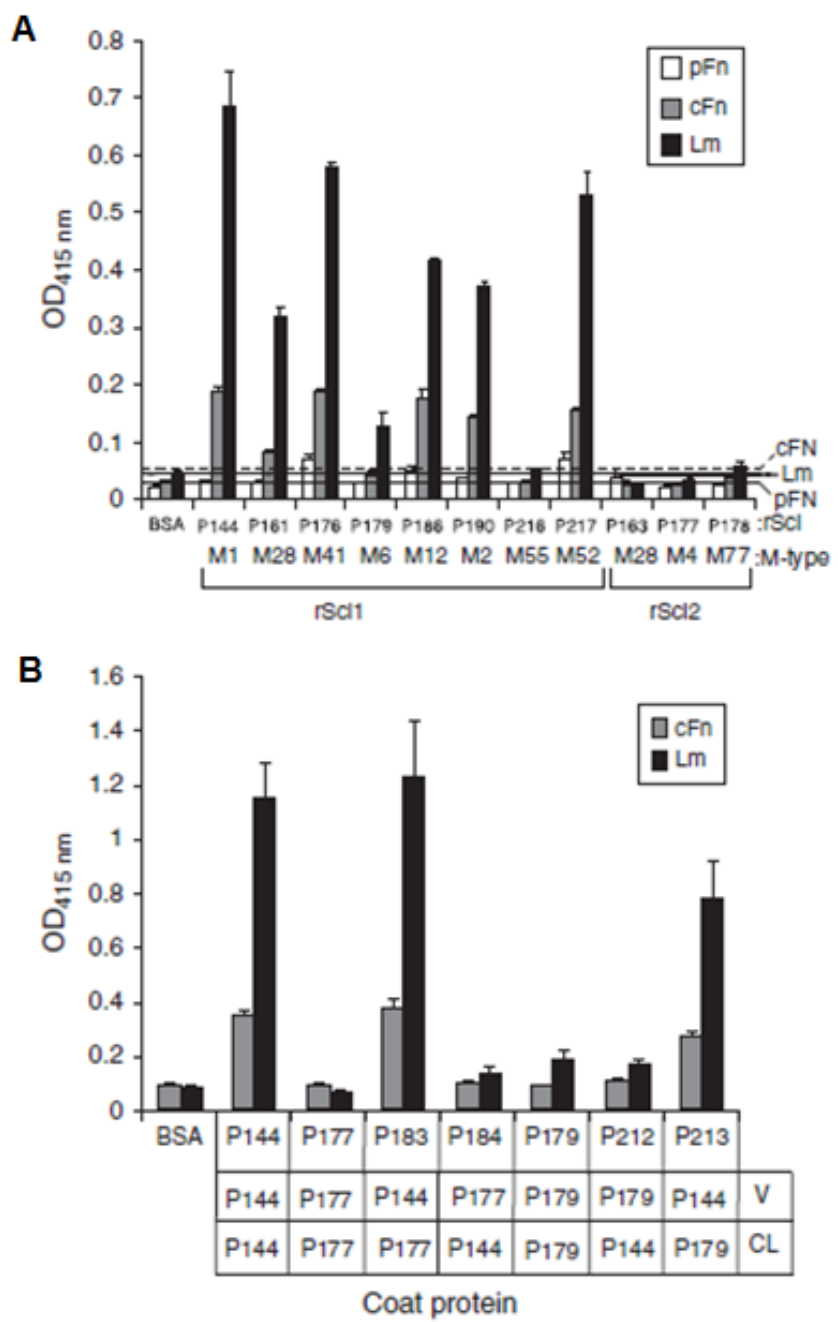

Figure 4. Characterization of ECM binding to rScl1 proteins. (a) Binding of $\mathrm{pFn}, \mathrm{cFn}$, and $\mathrm{Lm}$ to $\mathrm{rScl}$ proteins by ELISA. The designation of $\mathrm{rScl}$ constructs, as well as the GAS M-type from which each construct was derived (M-type), are indicated. The distinction between Scl1-derived (rScl1) and Scl2-derived (rScl2) proteins is shown. rScl- or BSA-bound ECM proteins were detected with specific antibodies. Graph bars represent the mean $\mathrm{OD}_{415} \pm \mathrm{SD}$ of triplicate wells from a single experiment that is representative of three independent experiments. The solid, dotted, and spaced lines represent binding of pFn, cFn, and Lm, respectively to the BSA-coated wells with average values plus 3 SD as negative controls. (b) Identification of the Scl1 domain responsible for ECM binding. Chimeric $\mathrm{rScl}$ proteins, which were constructed by domain swapping between ECM-binding-positive and ECM-binding-negative rScls identified in panel (a), were tested for binding to cFn and Lm by ELISA. Domain composition of each chimeric molecule is shown below the graph. Graph bars represent the mean $\mathrm{OD}_{415} \pm S D$ of triplicate wells from a single experiment that is representative of three independent experiments. 


\section{Chapter 3}

Contribution of the Streptococcal collagen-like protein-1 (Scl1) in Biofilm

Formation by Group A Streptococcus 


\title{
The streptococcal collagen-like protein-1 (Scl1) is a significant determinant for biofilm formation by group A Streptococcus
}

\author{
Heaven A Oliver-Kozup ${ }^{1}$, Meenal Elliott ${ }^{1}$, Beth A Bachert ${ }^{\ddagger}$, Karen H \\ Martin $^{2,3}$,Sean D Reid ${ }^{4}$, Diane E Schwegler-Berry ${ }^{5}$, Brett J Green ${ }^{6}$, \\ Slawomir Lukomski ${ }^{*}$
}

Address: ${ }^{1}$ Department of Microbiology, Immunology, and Cell Biology, ${ }^{2}$ Mary Babb Randolph Cancer Center and ${ }^{3}$ Microscope Imaging Facility, West Virginia University School of Medicine, Morgantown, WV, 26506; ${ }^{4}$ Department of Microbiology and Immunology, Wake Forest University School of Medicine, Winston-Salem, NC, 27157; and ${ }^{5}$ Pathology and Physiology Research Branch and ${ }^{6}$ Allergy and Clinical Immunology Branch, Health Effects Laboratory Division, National Institute of Occupational Safety and Health (NIOSH), Morgantown, WV, 26505, USA

Published in BMC Microbiology (2011) 11:262 


\section{Abstract}

Group A Streptococcus (GAS) is a human-specific pathogen responsible for a number of diseases characterized by a wide range of clinical manifestations. During host colonization GAS-cell aggregates or microcolonies are observed in tissues. GAS biofilm, which is an in vitro equivalent of tissue microcolony, has only recently been studied and little is known about the specific surface determinants that aid biofilm formation. In this study, we demonstrate that surface-associated streptococcal collagen-like protein-1 (Scl1) plays an important role in GAS biofilm formation. Biofilm formation by M1-, M3-, M28-, and M41-type GAS strains, representing an intraspecies breadth, were analyzed spectrophotometrically following crystal violet staining, and characterized using confocal and field emission scanning electron microscopy. The M41-type strain formed the most robust biofilm under static conditions, followed by M28- and M1type strains, while the M3-type strains analyzed here did not form biofilm under the same experimental conditions. Differences in architecture and cell-surface morphology were observed in biofilms formed by the M1- and M41-wild-type strains, accompanied by varying amounts of deposited extracellular matrix and differences in cell-to-cell junctions within each biofilm. Importantly, all Scl1negative mutants examined showed significantly decreased ability to form biofilm in vitro. Furthermore, the Scl1 protein expressed on the surface of a heterologous host, Lactococcus lactis, was sufficient to induce biofilm formation by this organism. Overall, this work (i) identifies variations in biofilm formation capacity among pathogenically different GAS strains, (ii) identifies GAS surface 
properties that may aid in biofilm stability and, (iii) establishes that the Scl1 surface protein is an important determinant of GAS biofilm, which is sufficient to enable biofilm formation in the heterologous host Lactococcus. In summary, the GAS surface adhesin Scl1 may have an important role in biofilm-associated pathogenicity. 


\section{Introduction}

Microbial biofilm formation is an important virulence mechanism, which allows immune evasion and survival against antibiotic treatments $(19,64)$. Many bacterial nosocomial infections are associated with biofilms formed on contaminated medical devices. Dispersal of biofilm has also been proposed to augment infection spread $(11,20,31,36,40,42)$. For group A Streptococcus (GAS), biofilm research is an emerging field and little is known about the specific surface determinants that aid in biofilm formation. GAS is characteristically associated with significant human morbidity and it is responsible for the clinically common superficial throat and skin infections, such as pharyngitis and impetigo, as well as invasive soft tissue and blood infections like necrotizing fasciitis and toxic shock syndrome (24). Although GAS biofilm has not been associated with implanted medical devices, tissue microcolonies of GAS encased in an extracellular matrix were demonstrated in human clinical specimens (1). Studies reported to date support the involvement of GAS surface components in biofilm formation, including the $\mathrm{M}$ and $\mathrm{M}$-like proteins, hyaluronic acid capsule, pili and lipoteichoic acid $(18,22,52)$. As shown by Cho and Caparon (18), multiple genes are upregulated during biofilm formation and development, including the streptococcal collagen-like protein-1 (Scl1).

The scl1 gene encoding the Scl1 protein has been found in every GAS strain investigated and its transcription is positively regulated by Mga $(2,3,46$, $47,60)$, indicating that Scl1 is co-expressed with a number of proven virulence 
factors. Structurally, the extracellular portion of Scl1 protein extends from the GAS surface as a homotrimeric molecule composed of distinct domains that include the most outward $\mathrm{N}$-terminal variable $(\mathrm{V})$ region and the adjacent collagen-like $(C L)$ region composed of repeating GlyXaaYaa (GXY) sequence. The linker (L) region is close to the cell surface and contains a series of conserved direct repeats. The Scl1 protein can bind selected human extracellular matrix components (17) and cellular integrin receptors $(15,16,37)$, as well as plasma components $(14,27,34,55,61)$.

In this study, we investigated the importance of Scl1 in GAS biofilm using defined isogenic wild-type and sc/1-inactivated mutant strains of GAS. We report that (i) the pathogenically diverse M41-, M28-, M3- and M1-type GAS wild-type strains have varying capacities to produce biofilm on an abiotic surface; (ii) Scl1 plays an important role during the main stages of biofilm formation with Scl1negative mutants having an abrogated capacity for adhesion, microcolony formation and biofilm maturation; and (iii) variations in surface morphology as well as in extracellular matrix associated with bacterial cells suggest two distinct but plausible mechanisms that potentially stabilize bacterial microcolonies. We additionally show that expression of Scl1 in Lactococcus lactis is sufficient to support a biofilm phenotype. Overall, this work reveals a significant role for the Scl1 protein as a cell-surface component during GAS biofilm formation among pathogenically varying strains. 


\section{Materials and Methods}

GAS strains and growth conditions. The wild-type GAS strains M41MGAS6183, M1- MGAS5005, and M28-type MGAS6143, as well as their scl1inactivated isogenic mutants and complemented M41 $\Delta s c / 1$ mutant have been previously described $(16,34,45)$. In addition, a set of the wild-type M3-type GAS strains MGAS158, MGAS274, MGAS315, MGAS335, MGAS1313, and MGAS2079 was also used. GAS cultures were routinely grown on brain-heart infusion agar (BD Biosciences) and in Todd-Hewitt broth (BD Biosciences) supplemented with $0.2 \%$ yeast extract (THY medium) at $37^{\circ} \mathrm{C}$ in an atmosphere of $5 \% \mathrm{CO}_{2}-20 \% \mathrm{O}_{2}$. Logarithmic phase cultures harvested at the optical density $(600 \mathrm{~nm})$ of about $0.5\left(\mathrm{OD}_{600} \sim 0.5\right)$ were used to prepare GAS inocula for biofilm analysis. Colony counts were verified by plating on tryptic soy agar with $5 \%$ sheep's blood (Remel). Lactococcus lactis subsp. cremoris strain MG1363 (provided by Dr. Anton Steen) were grown using M17 broth or agar media (Oxoid) supplemented with $0.5 \mathrm{M}$ sucrose and $0.5 \%$ glucose (SGM17 media) at $30^{\circ} \mathrm{C}$ in an atmosphere of $5 \% \mathrm{CO}_{2}-20 \% \mathrm{O}_{2}$.

\section{Heterologous Scl1 expression in Lactococcus lactis}

Lactococcus transformation. To obtain electrocompetent cells, $500 \mathrm{ml}$ of SGM17 broth supplemented with $2 \%$ glycine was inoculated with an overnight culture and grown until $\mathrm{OD}_{600} \sim 0.4$ was reached. Cells were harvested and washed twice with ice-cold solution A ( $0.5 \mathrm{M}$ sucrose, $10 \%$ glycerol); cells were 
then re-suspended in solution $A$ (1/1000 of original culture volume) and stored at $-80^{\circ} \mathrm{C}(35)$. For transformation, cells were thawed on ice and mixed with $1 \mu \mathrm{l}$ of DNA of the Scl1.41-expressing plasmid pSL230 or pJRS525-vector(16); and transferred to a cold 1-mm electrode-gap cuvette. Cells were pulsed with $2.0 \mathrm{kV}$ at $25 \mu \mathrm{F}$ and 400 ohms. Immediately following, suspensions were mixed with 1 $\mathrm{ml}$ outgrowth medium (SGM17 broth supplemented with $20 \mathrm{mM} \mathrm{MgCl} 2$ and $2 \mathrm{mM}$ $\mathrm{CaCl}_{2}$ ) and incubated for $2.5 \mathrm{~h}$ before plating on SGM17 agar supplemented with spectinomycin (59).

Molecular characterization of transformants. The pSL230 was detected in Lactococcus lactis MG1363 transformants by PCR amplification directly from bacterial colonies with scl1.41-gene specific primers 232up (5'CTCCACAAAGAGTGATCAGTC) and 232rev (5'TTAGTTGTTTTCTTTGCGTTT); pSL230 plasmid DNA was used as a positive control. PCR samples were analyzed on $1 \%$ agarose gel in Tris-acetate-EDTA buffer and stained with ethidium bromide. Inocula from colonies of L. lactis MG1363, as well as colonies harboring either pJRS525 vector or pSL230 construct were used in subsequent experiments.

Western blot analysis. Cell-wall extracts were prepared as previously described (16). Briefly, cells grown to $\mathrm{OD}_{600} \sim 0.4$ were harvested, washed with TES $(10 \mathrm{mM}$ Tris, 1mM EDTA, 25\% Sucrose), re-suspended in TES-LMR (TES containing $1 \mathrm{mg} / \mathrm{ml}$ hen egg lysozyme, $0.1 \mathrm{mg} / \mathrm{ml}$ mutanolysin, $0.1 \mathrm{mg} / \mathrm{ml}$ RNAseA and $1 \mathrm{mM}$ 
PMSF) and incubated at $37^{\circ} \mathrm{C}$ for $1 \mathrm{~h}$. After centrifugation at $2500 \mathrm{~g}$ for $10 \mathrm{~min}$, the supernatants were precipitated with ice-cold TCA $\left(16 \%\right.$ final) at $-20^{\circ} \mathrm{C}$ overnight. Precipitates were rinsed thoroughly with ice-cold acetone and dissolved in $1 \times$ sample buffer at $250 \mu$ per unit $\mathrm{OD}_{600}$. Samples were subjected to $10 \%$ SDS-PAGE, transferred to nitrocellulose, and probed with anti-P176 antiserum followed by goat anti-rabbit-HRP and detected employing chemiluminescent substrate (Pierce).

Flow cytometry. Bacterial cells were grown to mid-log phase $\left(\mathrm{OD}_{600} \sim 0.4\right)$, washed once with filtered DPBS containing 1\% FBS and re-suspended in the same buffer. Five million cells were incubated with 1:400 dilution of primary reagents, either rabbit pre-bleed (control) or rabbit anti-P176 antiserum for 30 min on ice, washed with DPBS-FBS and then incubated with 1:200 dilution of second reagent donkey anti-rabbit-APC (Jackson ImmunoResearch) for $30 \mathrm{~min}$ on ice. After a final wash and re-suspension in DPBS-FBS, flow cytometric data were acquired with FACSCaliber (BD Biosciences) and analyzed employing FCS Express (De Novo Software).

\section{Analysis of biofilm formation}

Crystal violet staining assay: Biofilm formation was tested using tissue culture treated polystyrene 24-well plates. $1.5 \mathrm{ml}$ of logarithmic-phase GAS or Lactococcus cultures were seeded without dilution into wells and incubated at $37^{\circ} \mathrm{C}$ for GAS and $30^{\circ} \mathrm{C}$ for Lactococcus in an atmosphere of $5 \% \mathrm{CO}_{2}-20 \% \mathrm{O}_{2}$ 
according to indicated time points upon which medium was aspirated. Wells were washed with PBS and $500 \mu \mathrm{l}$ of $1 \%$ crystal violet was added to each well, and incubated at room temperature for $30 \mathrm{~min}$. Dye was then aspirated, wells were washed with PBS, and stain was solubilized with $500 \mu \mathrm{l}$ of $100 \%$ ethanol. Spectrophotometric readings at $\mathrm{OD}_{600}$ were recorded for each sample per time point. Samples were analyzed in triplicate in at least three experiments.

Confocal Laser Scanning Microscopy (CLSM). To visualize GAS and L. lactis strains by CLSM, bacterial cells were transformed with a GFP-encoding plasmid, pSB027 (23). 15-mm glass cover slips were placed into 24-well tissue culture plate wells. Logarithmic-phase bacterial cultures were inoculated without dilution and grown for $24 \mathrm{~h}$. Cover slips were rinsed with PBS and fixed with 3\% paraformaldehyde at room temperature for $30 \mathrm{~min}$. Biofilms present on cover slips were washed with PBS and mounted onto slides using Prolong Gold mounting media (Invitrogen). Confocal images were acquired using a 63x/1.40 Plan-Apochromat objective and a Zeiss LSM 510 laser scanning confocal on an Axiolmager Z1 microscope. An orthogonal view of the Z-stacks was used to display and measure biofilm thickness using Zeiss LSM software. Ten representative images within a single experiment were used to calculate the average vertical thickness measured in micrometers.

To visualize extracellular matrix associated with GAS cells, $24 \mathrm{~h}$ biofilm samples were reacted with $100 \mu \mathrm{g}$ of tetramethyl rhodamine isothiocyanate(TRITC)-conjugated concanavalin A (TRITC-ConA) (Invitrogen) for $30 \mathrm{~min}$ at 
room temperature in the dark prior to mounting with Prolong Gold medium. An average of ten microscopic views within each sample was reviewed using the $63 x / 1.40$ objective, as described above.

Field emission scanning electron microscopy (FESEM). GAS biofilm samples were grown for $24 \mathrm{~h}$ on glass cover slips, washed with PBS, and fixed with $3 \%$ paraformaldehyde for $2 \mathrm{~h}$ and post-fixed in osmium tetroxide. Samples were next dehydrated in an ethanol gradient, dried using hexamethyldisalizane, mounted onto aluminum stubs and sputter-coated with gold/palladium. The samples were then imaged on a Hitachi S-4800 field emission scanning electron microscope.

Quantitation of hydrophobicity. A modified hexadecane method $(22,32,56)$ was used to determine the cell hydrophobicity. Briefly, $5 \mathrm{ml}$ of the logarithmicphase GAS or Lactococcus cultures $\left(\mathrm{OD}_{600} \sim 0.5\right)$ were pelleted, washed and resuspended in $5 \mathrm{ml}$ of PBS. One $\mathrm{ml}$ of hexadecane was added, vortexed for $1 \mathrm{~min}$ and incubated for $10 \mathrm{~min}$ at $30^{\circ} \mathrm{C}$. Mixtures were then vortexed for an additional 1 min and allowed to stand for 2 min for phase separation at room temperature. The absorbance of the lower aqueous phase was read at $\mathrm{OD}_{600}$ and compared against the PBS control. Actual hydrophobicity value was calculated using the following equation: Actual Value $=\left[1-\left(A / A_{\circ}\right)\right] \times 100$, where $A$ is $O D_{600}$ value after hexadecane treatment and $A_{\circ}$ is $O D_{600}$ prior to hexadecane treatment. 
Statistical analysis. Statistical significance was determined using a two-tailed paired Student's $t$-test. The results were considered statistically significant with $P$ $\leq 0.05\left(^{*}\right)$ and $P \leq 0.001\left(^{* *}\right)$. 


\section{Results}

Wild-type GAS strains have heterogeneous capacity for biofilm formation on abiotic surfaces. Biofilm formation was compared between M41-, M28-, M3and M1-type GAS strains representing distinct epidemiological traits (Figure 1). To assess biofilm formation after $24 \mathrm{~h}$, we used spectrophotometric measurements recorded following crystal violet staining (Figure 1A). Both the M41- and M28-type strains produced more biomass as compared with M1 strain. Furthermore, the M3-type strain produced the lowest absorbance values in a crystal violet assay, indicative of lower cell biomass, as compared with the other wild-type strains. These experiments confirm previous observations $(19,43)$ that GAS strains have varying capacity to form biofilm in vitro.

The failure of M3-type strain MGAS315 to produce substantial cellular biomass in the above assay was intriguing because sequence analysis of the Scl1.1 allele found in MGAS315 revealed the presence of a TAA stop codon in the $11^{\text {th }}$ GXY repeat of the Scl1.3-CL region containing a total of 25 GXY triplets (48). This premature stop codon results in a truncated Scl1.3 variant composed of 102 amino acids ( 11.4 kDa), which does not contain the cell wall-membrane (WM) associated region, thus, preventing it from anchoring to the bacterial cell surface (Figure 1B). This prompted us to investigate the biofilm formation by five additional M3-type strains, all harboring the same scl1.3 allele. Five additional M3-type strains, MGAS335, MGAS1313, MGAS2079, MGAS274 and MGAS158, all harboring the same scl1.3 allele (48) also produced poor biofilm under static 
conditions, as measured by crystal violet staining. Confocal laser scanning microscopy (CLSM) of three representative strains (MGAS315, MGAS2079, and MGAS158) corroborated results obtained from the crystal violet assay, indicating that these M3-type strains lack the ability to form appreciable biofilm structure. Our data suggest that the lack of capacity for biofilm-formation among M3-type GAS strains examined here might be correlated, at least in part, with lack of surface-attached Scl1.3 protein.

\section{Microscopic evaluation reveals differences in biofilm surface morphology.}

We next conducted microscopic analysis of the biofilms formed by the wild-type (WT) M41-, M28-, and M1-type GAS strains. First, we examined the overall structural characteristics of biofilms formed after $24 \mathrm{~h}$ using CLSM (Figure $4 \mathrm{D}$ F); Additional file 1: Figure S1 A-F). The average biofilm thickness (see Methods section) differed among all three strains with M1 producing considerably thinner biofilm (mean value of $9 \mu \mathrm{m})$ compared to M28 $(12 \mu \mathrm{m})$ and M41 (15 $\mu \mathrm{m})$, a result consistent with lower spectrophotometric absorbance values (Figure 1A). In addition to measured differences in biofilm thickness, closer examination of the $X-Y$ orthogonal Z-stack views, representing biofilm cross-sections, revealed architectural differences among the M41, M28, and M1 biofilms. The M1 biofilm, although the thinnest, seems to consist of densely-packed cells that form continuous layers, while the M28 and especially M41 biofilms seem to be less dense but exhibit more elevated supracellular assembly. We therefore used field emission scanning electron microscopy (FESEM) to define more accurately 
these supracellular differences observed by CLSM between the biofilms produced by the WT M1 and M41 GAS (Figure 2). FESEM exposed notable architectural differences between biofilms formed by these two strains. The M41 (Figure 2, panel A) biofilm was characterized by more diverse surface architecture with the evidence of depressions or crypts, whereas the M1 biofilm (panel B) seems to lack such pronounced surface characteristics. At higher magnification, the M41 cells have a studded cell surface morphology with protrusions linking both sister cells and cells in adjacent chains (panel c). In contrast, the M1 cells had a relatively smoother appearance likely due to the rich bacterial-associated extracellular matrix (BAEM) surrounding these cells and covering their surface (panel d). BAEM material, which was clearly seen at higher resolution between the M1-type cells, was not as evident between cells of the M41-type GAS.

\section{GAS biofilms differ in production of bacterial-associated extracellular} matrix. The production of BAEM has been shown to be an integral component in the structural integrity of a biofilm, imparting protection from dehydration, host immune attack, and antibiotic sensitivity $(26,39)$. GAS cells encased in a glycocalyx were first identified by Akiyama et al. in skin biopsies obtained from impetigo patients. We therefore compared the production of BAEM within biofilms employing GFP-expressing GAS strains of the M1 and M41 type (Figure 3). Cells were grown to form biofilms on glass cover slips for $24 \mathrm{~h}$ and stained with TRITCconcanavalin A (ConA), a fluorescently-labeled lectin that binds to the 
extracellular polysaccharides in biofilms (51). Fluorescent microscopy was performed to compare matrix production (red staining) by GAS strains (green). Visual screening of both biofilms indicated that the M41-type strain formed a more dispersed extracellular matrix as compared to the M1 strain, which had a dense, more closely associated matrix. In addition, averages of at least 10 fields of ConA stained matrix by CLSM support our FESEM observations that more BAEM is deposited within the biofilm by the M1 GAS cells as compared to M41 GAS. This is in agreement with the report from Akiyama et al that showed a substantial FITC-ConA stained matrix associated with T1-type GAS microcolonies in vivo and in vitro (1).

\section{Scl1 protein significantly contributes to biofilm formation by GAS.} Variations in GAS pathogenicity and capacity to form biofilm are driven by specific proteins and components present on the cell surface or are secreted by the organism. It has been shown that deletion of the $M$ and $M$-like surface proteins or capsule, as well as increased expression of the secreted SpeB protease decreases biofilm formation dramatically for some strains of GAS (13, $22,25)$. Therefore, we investigated the role of Scl1 in biofilm formation by comparing biofilms formed by GAS WT and sc/1-inactivated ( $\Delta s c / 1)$ mutant strains (Figure 4; Additional file 1: Figure S1 A-F). Bacterial biomass was evaluated spectrophotometrically following crystal violet staining at 1, 6, 12, and $24 \mathrm{~h}$ time points, representing different stages of biofilm formation, and absorbance values rendered for the WT and $\Delta s c / 1$ isogenic mutant strains were 
compared. The M41 $\Delta s c / 1$ mutant showed a $29-35 \%$ decrease in biofilm formation (the $\mathrm{OD}_{600}$ value obtained for the WT strain at each time point was considered 100\%), which was sustained throughout all time points. This reduction was statistically significant at initial adherence $(1 \mathrm{~h})$, as well as during biofilm development (6-12 h) and at maturation (24 h) (Figure $4 \mathrm{~A} ; P \leq 0.05$ at 1 and $12 \mathrm{~h}, P \leq 0.001$ at 6 and $24 \mathrm{~h}$ ). Complementation of Scl1.41 expression in the M41 $\Delta s c / 1$ mutant $(M 41 \mathrm{C}$ ) restored its ability to form biofilm to WT levels. Similarly, the M28 $\Delta s c / 1$ mutant had a significantly decreased capacity for biofilm formation in the range of $29-44 \%$ as compared to WT strain (Figure 4B; $P \leq 0.05$ at 1 and $6 \mathrm{~h}, P \leq 0.001$ at 3,12 and $24 \mathrm{~h}$ ). Likewise, there was a statistically significant decrease in $\mathrm{M} 1 \Delta s c / 1$ biofilm biomass by $\sim 42-75 \%$ compared to the WT strain (Figure 4C; $P \leq 0.001$ at $1-24 \mathrm{~h}$ ). CLSM analysis of corresponding 24-h biofilms of these strains confirmed our crystal violet staining results at $24 \mathrm{~h}$. The $\Delta s c / 1$ mutants had substantially decreased average biofilm thickness by more than $50 \%$ (mean values) as compared to the parental WT organisms (Figure 4DF). While these low average biofilm thickness values measured for the M1 $\Delta s c / 41$ $(6 \mu \mathrm{M})$ and $\mathrm{M} 28 \Delta s c / 1(5 \mu \mathrm{M})$ correspond to residual biofilms made by those mutants (Figure S1 A-D), by comparison, the M1 $\Delta s c / 1(4 \mu \mathrm{M})$ was shown not to produce a continuous biofilm layer under these conditions (Figure S1 E-F). Our data support the hypothesis that the Scl1 protein plays an important functional role during GAS biofilm formation and that Scl1 contribution varies among GAS strains with different genetic backgrounds. 
Scl1 expression affects surface hydrophobicity. The surface hydrophobicity of GAS has been shown to influence the adherence to abiotic surfaces. The presence of pili (52), M and M-like proteins, and lipoteichoic acid contributes to cell surface hydrophobic properties $(22,66)$, which in turn may influence biofilm formation by GAS. Here, we have investigated the contribution of Scl1 to surface hydrophobicity of M41-, M28-, and M1-type GAS strains using a modified hexadecane binding assay $(22,33,56)$. As shown in Table 1, the M28-type GAS strain MGAS6143 gave the highest actual hydrophobicity value of $94.3 \pm 0.73$, followed by the M41-type strain MGAS6183 (92.6 \pm 0.86$)$. In contrast, the overall surface hydrophobicity of the M1-type GAS strain MGAS5005 (80.3 \pm 0.89$)$ was significantly lower compared to both M28 and M41 strains $(P \leq 0.001$ for each comparison). Inactivation of sc/1.41 in M41-type GAS resulted in a modest, although statistically significant, reduction in the hydrophobicity index $(100 \%$ for WT vs. $92 \%$ for mutant, $P \leq 0.001)$. In-trans complementation of the Scl1.41 expression in M41 $\Delta s c / 1-C$ restored the hydrophobic phenotype of the cells to WT level (hydrophobicity index $\sim 105 \%$ ). In comparison, the contribution of the Scl1.1 and Scl1.28 proteins to surface hydrophobicity is more substantial, as evidenced by a $\sim 21 \%$ and $\sim 22 \%$ reduction of the hydrophobicity indices of the mutants as compared to the corresponding WT strains, respectively ( $P \leq 0.001$ for both). Thus, the Scl1-mediated GAS-cell surface hydrophobicity reported here may contribute to the ability of this organism to form biofilm, as suggested for other cell surface components $(22,66)$. 
Scl1 is sufficient to support biofilm formation in Lactococcus lactis. To assess whether Scl1 expression is sufficient to confer the ability for biofilm formation, we chose to express this protein in a heterologous L. lactis system (44, 57). The wild-type L. lactis strain MG1363 was transformed with plasmid pSL230 encoding the Scl1.41 protein (16) or with the shuttle vector pJRS525 alone. As shown in Figure 5A, PCR amplification of the sc/1.41 gene employing specific primers yielded no product from the WT L. lactis MG1363 (lane 1) and the MG1363::pJRS525 transformant (lane 2). A product of the expected size of $1.4 \mathrm{~kb}$ was amplified from the pSL230 plasmid DNA control (lane 4,) as well as was amplified from the MG1363::pSL230 transformant (lane 3). Surface expression of Scl1.41 was confirmed by immunoblot analysis of cell-wall extracts prepared from L. lactis WT, and the MG1363::pJRS525 and MG1363::pSL230 transformants, as well as MGAS6163 (WT M41 GAS). As shown in Figure 5B, rabbit antiserum raised against purified recombinant Scl1.41 protein P176 lacking the WM region detected the corresponding immunogen (lane 1), and the homologous full length protein in cell-wall extracts of MGAS6183 (lane 5) as well as MG1363::pSL230 L. lactis transformant (lane 4). This band was absent in cellwall extracts prepared from the WT L. lactis MG1363 (lane 2) and MG1363::pJRS525 transformant (lane 3). Expression of Scl1.41 at the cell surface was further established by flow cytometry. Rabbit anti-p176 antibodies stained Scl1.41 MG1363::pSL230 transformant, confirming the expression of Scl1.41 protein at the cell surface in the heterologous host L. lactis (Figure $5 \mathrm{C}$, 
red trace). This protein was absent at the surface of WT MG1363 (black trace) and MG1363::pJRS525 transformant (green trace).

The capacity of L. lactis expressing Scl1.41 to form biofilm was evaluated spectrophotometrically following crystal violet staining. As shown in Figure 5 D, the MG1363::pSL230 transformant demonstrated a significant increase in biofilmassociated biomass at $24 \mathrm{~h}$, as compared to wild type L. lactis or L. lactiscontaining pJRS525 vector $(P \leq 0.001)$. Crystal violet stained wells were photographed for visual representation of biofilm formation prior to spectrophotometric assay. Biofilm thickness and architecture were evaluated by CLSM (Figure 5E; Additional file 1: Figure S2 A-C). The MG1363::pSL230 transformant produced a substantially thicker biofilm $(14 \mu \mathrm{m})$ as compared to both MG1363 WT (6 $\mu \mathrm{m})$ and the vector-only transformant MG1363::pJRS525 (6 $\mu \mathrm{m})$. The MG1363::pSL230 cells formed highly aggregated structures, thus, acquiring a phenotype consistent with biofilm formation. As shown in Table 2, the MG1363::pSL230 transformant, expressing Scl1.41 surface protein, had significantly enhanced cell surface hydrophobicity (hydrophobicity index of $\sim 137 \%$ vs. $100 \%$ WT, $P \leq 0.001$ ) with an actual value of $82.0 \pm 2.6$, when compared to the MG1363 WT (59.7 \pm 7.2) and the vector-only MGAS1363::pJRS525 control (56.6 \pm 5.5). These data suggest a direct relationship between Scl1 expression and cell surface hydrophobicity and establish their involvement in the microorganism's ability to form biofilm in vitro. 


\section{Discussion}

Group A Streptococcus strains vary because of the vast number of M-protein types, and this variation is associated with varying frequency of isolation and exacerbation of disease $(10,67)$. The M41-, M28-, M3-, and M1-type strains selected for the current study represent a significant intraspecies diversity among clinical isolates of GAS. M41 GAS was a major causative agent of superficial skin infections $(4,5,65)$, and strain MGAS6183, harboring the Scl1.41 protein, has been studied extensively (15-17). M28-type GAS (strain MGAS6143) has historically been associated with puerperal fever and currently is responsible for extensive human infections world-wide (30). M1T1 GAS, represented by strain MGAS5005, is a globally disseminated clone responsible for both pharyngitis and invasive infections $(6,7,53)$. The M3-type strains of GAS cause a disproportionally large number of invasive GAS infections that are responsible for traumatic morbidity and death $(41,63)$.

Initial studies by Lembke et al. that characterized biofilm formation among various M types of GAS typically included several strains of the same M type (19, 43). These studies reported a significant strain-to-strain variation in ability to form biofilms within each $\mathrm{M}$ type. Studies that followed compared biofilm formation by defined isogenic WT and mutant strains to assess the contribution of specific GAS surface components responsible for a biofilm phenotype, including $M$ and M-like proteins, hyaluronic acid capsule, lipoteichoic acid, and pili $(22,52)$. In the 
current study, we have assessed the role and contribution of the surface protein Scl1 in the ability to support biofilm formation by GAS strains of four distinct M types.

Recent advances in molecular mega- and pathogenomics has enabled the characterization of numerous M3-type strains with a single nucleotide resolution $(8,9)$. Interestingly, all five M3-type strains MGAS158, 274, 315, 335, and 1313 that were originally used for sc/1-gene sequencing (46), plus an additional strain MGAS2079 (not reported) harbor the same scl1.3 allele containing a null mutation that would result in secretion of a truncated Scl1.3-protein variant. Here, we demonstrate that these GAS strains do not form biofilm on an abiotic surface. Recently, bioinformatic screening of the sequences of 250 invasive M3-type strains isolated globally, has led to the detection of this single nucleotide polymorphism that results in disruption of Scl1.3 protein (Steve Beres and Jim Musser, personal communication). Lembke et al. reported heterogeneous biofilm formation among four M3-type GAS strains examined over a 24, 48, and 72-hour period (43). Biofilm was detected for one strain at a $48 \mathrm{~h}$ time point, on a fibrinogen-coated surface; however, it is not known whether this clinical isolate forms biofilm on abiotic surface, whether it expresses the truncated or full-length Scl1.3 protein, and whether it produces an unknown fibrinogen-binding protein, which could augment the attachment and biofilm formation. Therefore, additional studies are necessary to define the contributions of other biofilm-formation determinants in M3-type strains. 
Inasmuch as, variation in biofilm formation among GAS isolates of the same Mtype has been established, the molecular basis of this phenotypic variation is not known. Several GAS surface-associated and secreted components were shown to contribute to variation in biofilm $(22,25,52)$. In addition, transcription regulators, such as Mga, CovR, and Srv are likely to play substantial roles in GAS biofilm formation $(18,25)$ due to their transcriptional regulation of numerous genes. Therefore, it is logical to assume that the combination of genomic/proteomic make up, allelic polymorphisms, and transcription regulation all contribute to this phenomenon. In addition, discrepancies between in vitro data obtained with laboratory-stored strains and microcolony formation in vivo likely exist and add yet another unknown to the complexity of GAS biofilm/microcolony formation and its role in pathogenesis. Despite this complexity, the analyses involving isogenic strains of the same genetic background provide valuable information that allows assessment of the role and contribution of a given GAS component to biofilm formation.

The M1 MGAS5005 strain was shown to form biofilm in vitro and in experimental animals $(20,25,62)$, and the present study demonstrates a significant role of Scl1.1 in this process. Likewise, the MGAS6183 strain, representing M41-type isolates often associated with pyoderma, produced a more robust biofilm biomass under the same experimental conditions and Scl1.41-deficient mutant was found to be an important determinant in this process. Similarly, Scl1.28 protein significantly contributes to a robust biofilm made by the M28-type strain MGAS6143. However, a recent study reported that 
another surface protein, designated AspA, found in M28-type GAS significantly contributed to biofilm formation (50). The $\triangle a s p A$ isogenic mutant showed $60 \%$ reduction in biofilm formation. The strain MGAS6180, which they used, expresses the same Scl1.28 variant present in the MGAS6143 strain we used; our Scl1.28 mutant showed $\sim 44 \%$ reduction in $24 \mathrm{~h}$ biofilm. We propose that several surface proteins contribute to biofilm formation by M28-type strains including proteins AspA and Scl1.28, and potentially, proteins F1/Sfbl and F2 that are also present in these strains (16). This redundancy is likely responsible for the observed residual biofilms produced by the AspA- and Scl1.28-deficient mutants.

The observed heterogeneity in biofilm architecture of different GAS strains was previously observed by Lembke et al. (43) and was also documented in the current study using FESEM. In addition, here we report the differences in GAScell surface morphology and within cell-to-cell junctions in biofilms formed by M1and M41-type strains. The structural and genetic determination of these differences is not known since M41 genome has not been sequenced, but may be associated with the presence of additional surface proteins, such as the F2 protein (38) encoded by prtf2 gene found in this strain (16). Even more striking was an observed difference in the amount of the extracellular material associated with each strain, referred to as BAEM (bacteria-associated extracellular matrix). It has been shown that extracellular matrix, also called glycocalyx, is produced by biofilm-forming bacteria. DNA, lipids, proteins (25), polysaccharides and dead cell debris (12) were identified in this matrix and for gram-positive bacteria, 
teichoic acids have also been detected $(29,54)$. The exopolysaccharide component of the glycocalyx is detected using carbohydrate-binding lectins, such as concanavalin A (ConA) (1). Both FESEM analysis and ConA staining detected more BAEM associated with M1 biofilm compared to M41, which produced larger biofilm. These observations suggest that GAS biofilm is stabilized differently by different strains and that higher BAEM production does not necessarily predetermine larger biofilm mass. Consequently, a combination of biofilm features rather than biofilm size alone may be more relevant to pathogenicity of a given GAS strain.

Diminished adherence and biofilm formation could be associated with changes in cell surface hydrophobicity (21) of the scl1 mutants. Indeed, the lack of Scl1 resulted in both decreased hydrophobicity and the ability to form biofilm, albeit in a somewhat disproportionate manner. A decrease in the hydrophobicity index by only $\sim 8 \%$, as compared to the wild type-strain, was measured for the M41 $\Delta s c / 1$ mutant and this modest decrease was accompanied by a rather large reduction in biofilm formation capacity after $24 \mathrm{~h}$ by $30 \%$. Greater decrease in cell-surface hydrophobicity was measured for the M1 $\Delta s c / 1$ ( 21\%) and M28 $\Delta s c / 1$ ( 22\%) mutants, which was accompanied by a significant loss in biofilm formation after $24 \mathrm{~h}$ by both isogenic strains by $\sim 55 \%$ and $\sim 41 \%$ ( $P \leq 0.001$ for each comparison), respectively. In addition, heterologous expression of Scl.41 in L. lactis increased hydrophobicity index of this organism to $\sim 137 \%$ of the WT level, which was accompanied by significant increase in its ability to form biofilm. Similar observations have been reported for the $\mathrm{M}$ and $\mathrm{M}$-like protein mutants 
that typically, but not always, exhibit concurrent loss of both biological features (22). For example, isogenic $\Delta$ Mrp49 mutant had a non-significant drop in hydrophobicity ( 2\%) but significantly lower biofilm formation after 48 h by $\sim 30 \%$, whereas $\Delta$ Emm1 mutant lost $\sim 78 \%$ hydrophobicity and $\sim 44 \%$ biofilm formation capacity. In summary: (i) here we report that the Scl1 adhesin is also a hydrophobin with varying contribution to the overall surface hydrophobicity among GAS strains representing different $M$ types and (ii) Scl1-associated surface hydrophobicity is likely to contribute to Scl1-mediated biofilm formation.

To test whether Scl1 alone could support biofilm formation, we used a heterologous $L$. lactis strain, which provides an expression system for membrane-bound proteins of gram- positive bacteria with LPXTG cell-wall anchoring motifs $(28,44,49,59)$, including the group A streptococcal M6 protein $(57,58)$. In a recent study by Maddocks et al. (50) it was shown that heterologous expression of AspA GAS surface protein was able to induce a biofilm phenotype in L. lactis MG1363. We were also able to achieve a gain-offunction derivative of the L. lactis WT MG1363 strain, (MG1363::pSL230), displaying an altered phenotype associated with biofilm formation, as compared to wild-type parental and vector-only controls. These data support our current model that Scl1 protein is an important determinant of GAS biofilm formation.

As shown by crystal violet staining and CLSM, biofilm formation by the Scl1-negative mutants was compromised during the initial stage of adherence, as well as microcolony stabilization and maturation. Consequently, their capacity for biofilm formation as compared to the respective WT controls was greatly 
reduced. This comparison identifies for the first time that the Scl1 protein contributes significantly to biofilm assembly and stability. Based on these observations, as well as previous work by us and others, we propose the following model of Scl1 contribution to GAS tissue microcolony formation (Figure 6). First, the Scl1 hydrophobin (current study) initiates bacterial adhesion to animate surfaces within the host (21). Next, the Scl1 adhesin anchors the outside edge of growing microcolony in tissue by direct binding to tissue extracellular matrix components, cellular fibronectin and laminin (17). Microcolony development is stabilized by Scl1-Scl1 scaffolding resulting from Scl1's capacity to form head-to-head dimers (68) between molecules located on adjacent chains. This model will be tested experimentally in future studies.

\section{Conclusions}

In the present work, using pathogenically differing GAS strains, we have demonstrated three concepts. First, we have confirmed previous observations that biofilm formation is an innate property of GAS strains. The M41-type strain used formed a more robust biofilm when compared to M28-type strain as well as M1-type strain. Importantly, the highly invasive M3-type strains devoid of the surface-associated Scl1 also lack the ability to form biofilm. Secondly, the absence of surface-associated Scl1 decreases GAS-cell hydrophobicity suggesting that Scl1 plays a role on the GAS surface as a hydrophobin. Thirdly, we have established that the Scl1 protein is a significant determinant for GAS biofilm formation. This concept was further tested by the heterologous expression 
of Scl1 in Lactococcus, an organism found in dairy fermentation environments, enabling it to form biofilm. Altogether, these data underscore the importance of Scl1 in biofilm-associated regulation of GAS pathogenicity. Recently published work has shown that the recombinant Scl1 binds to the extracellular matrix components, cellular fibronectin and laminin (17). Our current research provides a foundation warranting additional investigation as to whether direct Scl1-ECM binding may promote GAS biofilm as a bridging mechanism within host tissues. 


\section{Authors Contributions}

$\mathrm{HO}-\mathrm{K}$ is responsible for majority of experiments. ME characterized heterologous expression of Scl1 and BB characterized biofilm formation by M3-type strains. KHM assisted in biofilm analysis using CLSM. DS-B, BJG and HO-K performed FESEM imaging and analysis. SDR provided preliminary results and participated in helpful discussions. SL was the project leader and participated in overall design and coordination of the project. $\mathrm{HO}-\mathrm{K}$ and SL drafted the manuscript. All authors have read and approved the final manuscript.

\section{Acknowledgements}

We would like to thank Dr. Steen for providing the Lactococcus lactis subsp. cremoris strain MG1363. This work was supported in part by National Institutes and Health Grant AI50666 and by a research grant (RFDG) from the West Virginia University Research and Graduate Education (to S. L.). H. Oliver-Kozup was supported by a grant from the West Virginia Graduate Student Fellowship in Science, Technology, Engineering and Mathematics (STEM). Confocal microscopy experiments were performed in the West Virginia University Microscope Imaging Facility, which is supported in part by the Mary Babb Randolph Cancer Center and NIH grant P20 RR016440. We would like to acknowledge the assistance of the West Virginia University Flow Cytometry core facility, which was supported in part by a grant P30 RR032138 from the National Institutes of Health. The findings and conclusions in this report are those of the 
authors and do not necessarily represent the views of the National Institute of Occupational Safety and Health. 


\section{References}

1. Akiyama, H., S. Morizane, O. Yamasaki, T. Oono, and K. Iwatsuki. 2003. Assessment of Streptococcus pyogenes microcolony formation in infected skin by confocal laser scanning microscopy. J Dermatol Sci 32:193-199.

2. Almengor, A. C., and K. S. Mclver. 2004. Transcriptional activation of sclA by Mga requires a distal binding site in Streptococcus pyogenes. J Bacteriol 186:7847-7857.

3. Almengor, A. C., M. S. Walters, and K. S. Mclver. 2006. Mga is sufficient to activate transcription in vitro of sof-sfbX and other Mga-regulated virulence genes in the group $A$ Streptococcus. J Bacteriol 188:2038-2047.

4. Anthony, B. F. 2000. Streptococcal pyoderma, p. 144-151. In D. L. Stevens and E. L. Kaplan (ed.), Streptococcal infections. Oxford University Press, New York, N. Y.

5. Anthony, B. F., L. V. Perlman, and L. W. Wannamaker. 1967. Skin linfections and acute nephritis in American Indian children. Pediatrics 39:263-279.

6. Aziz, R., and M. Kotb. 2008. Rise and persistence of global M1T1 clone of Streptococcus pyogenes. Emerg Infect Dis 14:1511-1517.

7. Aziz, R. K., R. A. Edwards, W. W. Taylor, D. E. Low, A. McGeer, and M. Kotb. 2005. Mosaic prophages with horizontally acquired genes account for the emergence and diversification of the globally disseminated M1T1 Clone of Streptococcus pyogenes. J Bacteriol 187:3311-3318.

8. Beres, S. B., G. L. Sylva, K. D. Barbian, B. Lei, J. S. Hoff, N. D. Mammarella, M.-Y. Liu, J. C. Smoot, S. F. Porcella, L. D. Parkins, D. S. Campbell, T. M. Smith, J. K. McCormick, D. Y. M. Leung, P. M. Schlievert, and J. M. Musser. 2002. Genome sequence of a serotype M3 strain of group A Streptococcus: Phage-encoded toxins, the high-virulence phenotype, and clone emergence. Proc Nat Acad Sci USA 99:10078-10083.

9. Beres, S. B., G. L. Sylva, D. E. Sturdevant, C. N. Granville, M. Liu, S. M. Ricklefs, A. R. Whitney, L. D. Parkins, N. P. Hoe, G. J. Adams, D. E. Low, F. R. DeLeo, A. McGeer, and J. M. Musser. 2004. Genome-wide molecular dissection of serotype M3 group A Streptococcus strains causing two epidemics of invasive infections. Proc Nat Acad Sci USA 101:11833-11838.

10. Bessen, D. E., C. M. Sotir, T. L. Readdy, and S. K. Hollingshead. 1996. Genetic corelates of throat and skin isolates of group A streptococci. J Infect Dis 173:896-900.

11. Boles, B. R., M. Thoendel, and P. K. Singh. 2005. Genetic variation in biofilms and the insurance effects of diversity. Microbiology 151:2816-2818.

12. Branda, S. S., J. E. Gonzalez-Pastor, E. Dervyn, S. D. Ehrlich, R. Losick, and R. Kolter. 2004. Genes involved in formation of structured multicellular communities by Bacillus subtilis. J Bacteriol 186:3970-3979.

13. Buznach, N., R. Dagan, and D. Greenberg. 2005. Clinical and bacterial characteristics of acute bacterial conjunctivitis in children in the antibiotic resistance era. Pediatr Infect Dis J 24:823-828.

14. Caswell, C., R. Han, K. Hovis, P. Ciborowski, D. Keene, R. Marconi, and S. Lukomski. 2008. The Scl1 protein of M6-type group A Streptococcus binds the human complement regulatory protein, factor $\mathrm{H}$, and inhibits the alternative pathway of complement. Mol Microbiol 67:584-596.

15. Caswell, C. C., M. Barczyk, D. R. Keene, E. Lukomska, D. E. Gullberg, and S. Lukomski. 2008. Identification of the first prokaryotic collagen sequence motif that mediates 
binding to human collagen receptors, integrins $\alpha_{2} \beta_{1}$ and $\alpha_{11} \beta_{1}$. J Biol Chem 283:3616836175.

16. Caswell, C. C., E. Lukomska, N. S. Seo, M. Höök, and S. Lukomski. 2007. Scl1-dependent internalization of group A Streptococcus via direct interactions with the $\alpha_{2} \beta_{1}$ integrin enhances pathogen survival and re-emergence. Mol Microbiol 64:1319-1331.

17. Caswell, C. C., H. Oliver-Kozup, R. Han, E. Lukomska, and S. Lukomski. 2010. Scl1, the multifunctional adhesin of group A Streptococcus, selectively binds cellular fibronectin and laminin, and mediates pathogen internalization by human cells. FEMS Microbiol Lett 303:61-68.

18. Cho, K., and M. Caparon. 2005. Patterns of virulence gene expression differ between biofilm and tissue communities of Streptococcus pyogenes. Mol Microbiol 57:15451556.

19. Conley, J., M. E. Olson, L. S. Cook, H. Ceri, V. Phan, and H. D. Davies. 2003. Biofilm formation by group a streptococci: is there a relationship with treatment failure? J Clin Microbiol 41:4043-4048.

20. Connolly, K. L., A. L. Roberts, R. C. Holder, and S. D. Reid. 2011. Dispersal of Group A streptococcal biofilms by the cysteine protease SpeB leads to increased disease severity in a murine model. PLoS ONE 6:e18984.

21. Courtney, H. S., J. B. Dale, and D. L. Hasty. 2000. Strategies for preventing group A streptococcal adhesion and infection, p. 553-579. In Y. H. An and R. J. Friedman (ed.), Handbook of bacterial adhesion: principles, methods, and applications. Humana Press, Inc., Totowa, N. J.

22. Courtney, H. S., I. Ofek, T. Penfound, V. Nizet, M. A. Pence, B. Kreikemeyer, A. Podbielski, D. L. Hasty, and J. B. Dale. 2009. Relationship between expression of the family of $\mathrm{M}$ proteins and lipoteichoic acid to hydrophobicity and biofilm formation in Streptococcus pyogenes. PLoS ONE 4:e4166.

23. Cramer, T., Y. Yamanishi, B. E. Clausen, I. Forster, R. Pawlinski, N. Mackman, V. H. Haase, R. Jaenisch, M. Corr, V. Nizet, G. S. Firestein, H. P. Gerber, N. Ferrara, and R. S. Johnson. 2003. HIF-1 $\alpha$ is essential for myeloid cell-mediated inflammation. Cell 112:645-657.

24. Cunningham, M. W. 2000. Pathogenesis of group A streptococcal infections. Clin Microbiol Rev 13:470-511.

25. Doern, C. D., A. L. Roberts, W. Hong, J. Nelson, S. Lukomski, W. E. Swords, and S. D. Reid. 2009. Biofilm formation by group A Streptococcus: a role for the streptococcal regulator of virulence (Srv) and streptococcal cysteine protease (SpeB). Microbiology 155:46-52.

26. Donlan, R. M. 2002. Biofilms: microbial life on surfaces. Emerg Infect Dis 8:881-890.

27. Gao, Y., C. Liang, R. Zhao, S. Lukomski, and R. Han. 2010. The Scl1 of M41-type group A Streptococcus binds the high-density lipoprotein. FEMS Microbiol Lett 309:55-61.

28. Gerber, S. D., and M. Solioz. 2007. Efficient transformation of Lactococcus lactis IL1403 and generation of knock-out mutants by homologous recombination. J Basic Microb 47:281-286.

29. Gotz, F. 2002. Staphylococcus and biofilms. Mol. Microbiol. 43:1367-1378.

30. Green, N. M., S. B. Beres, E. A. Graviss, J. E. Allison, A. J. McGeer, J. Vuopio-Varkila, R. B. LeFebvre, and J. M. Musser. 2005. Genetic diversity among type emm28 group A Streptococcus strains causing invasive infections and pharyngitis. J Clin Microbiol 43:4083-4091. 
31. Grivet, M., J. J. Morrier, G. Benay, and O. Barsotti. 2000. Effect of hydrophobicity on in vitro streptococcal adhesion to dental alloys. J Mater Sci Mater Med 11:637-642.

33. Grivetti, L. E., and B. M. Ogle. 2000. Value of traditional foods in meeting macro- and micronutrient needs: the wild plant connection. Nutr Res Rev 13:31-46.

34. Han, R., C. C. Caswell, E. Lukomska, D. R. Keene, M. Pawlowski, J. M. Bujnicki, J. K. Kim, and S. Lukomski. 2006. Binding of the low-density lipoprotein by streptococcal collagenlike protein Scl1 of Streptococcus pyogenes. Mol Microbiol 61(2):351-67.

35. Holo, H., and I. F. Nes. 1989. High-frequency transformation, by electroporation, of Lactococcus lactis subsp. cremoris grown with glycine in osmotically stabilized media. Appl Environ Microbiol 55:3119-3123.

36. Horacek, B. M., J. W. Warren, A. Albano, M. A. Palmeri, J. C. Rembert, J. C. Greenfield, Jr., and G. S. Wagner. 2006. Development of an automated selvester scoring system for estimating the size of myocardial infarction from the electrocardiogram. J Electrocardiol 39:162-168.

37. Humtsoe, J. O., J. K. Kim, Y. Xu, D. R. Keene, M. Höök, S. Lukomski, and K. K. Wary. 2005. A streptococcal collagen-like protein interacts with the $\alpha_{2} \beta_{1}$ integrin and induces intracellular signaling. J Biol Chem 280:13848-13857.

38. Jaffe, J., S. Natanson-Yaron, M. G. Caparon, and E. Hanski. 1996. Protein F2, a novel fibronectin-binding protein from Streptococcus pyogenes, possesses two domains. Mol Microbiol 21:373-384.

39. Kania, R. E., G. E. Lamers, M. J. Vonk, P. T. Huy, P. S. Hiemstra, G. V. Bloemberg, and J. J. Grote. 2007. Demonstration of bacterial cells and glycocalyx in biofilms on human tonsils. Arch Otolaryngol Head Neck Surg 133:115-121.

40. Kaplan, J. B., M. F. Meyenhofer, and D. H. Fine. 2003. Biofilm growth and detachment of Actinobacillus actinomycetemcomitans. J Bacteriol 185:1399-1404.

41. Kaul, R., A. McGeer, D. E. Low, K. Green, B. Schwartz, O. G. A. S. Study, and A. E. Simor. 1997. Population-based surveillance for group A streptococcal necrotizing fasciitis: clinical features, prognostic indicators, and microbiologic analysis of seventy-seven cases. Am. J. Med. 103:18-24.

42. Lauderdale, K. J., C. L. Malone, B. R. Boles, J. Morcuende, and A. R. Horswill. 2010. Biofilm dispersal of community-associated methicillin-resistant Staphylococcus aureus on orthopedic implant material. J Orthop Res 28:55-61.

43. Lembke, C., A. Podbielski, C. Hidalgo-Grass, L. Jonas, E. Hanski, and B. Kreikemeyer. 2006. Characterization of biofilm formation by clinically relevant serotypes of group $A$ streptococci. Appl Environ Microbiol 72:2864-2875.

44. Linares, D. M., J. Kok, and B. Poolman. 2010. Genome Sequences of Lactococcus lactis MG1363 (Revised) and NZ9000 and Comparative Physiological Studies. J. Bacteriol. 192:5806-5812.

45. Lukomski, S., N. P. Hoe, I. Abdi, J. Rurangirwa, P. Kordari, M. Liu, S. J. Dou, G. G. Adams, and J. M. Musser. 2000. Nonpolar inactivation of the hypervariable streptococcal inhibitor of complement gene (sic) in serotype M1 Streptococcus pyogenes significantly decreases mouse mucosal colonization. Infect Immun 68:535-542.

46. Lukomski, S., K. Nakashima, I. Abdi, V. J. Cipriano, R. M. Ireland, S. D. Reid, G. G. Adams, and J. M. Musser. 2000. Identification and characterization of the scl gene encoding a group A Streptococcus extracellular protein virulence factor with similarity to human collagen. Infect Immun 68:6542-6553.

47. Lukomski, S., K. Nakashima, I. Abdi, V. J. Cipriano, B. J. Shelvin, E. A. Graviss, and J. M. Musser. 2001. Identification and characterization of a second extracellular collagen-like 
protein made by group A Streptococcus: control of production at the level of translation. Infect Immun 69:1729-1738.

48. Lukomski, S., S. Sreevatsan, C. Amberg, W. Reichardt, M. Woischnik, A. Podbielski, and J. M. Musser. 1997. Inactivation of Streptococcus pyogenes extracellular cysteine protease significantly decreases mouse lethality of serotype M3 and M49 strains. J Clin Invest 99:2574-2580.

49. Luo, H. L., K. Wan, and H. H. Wang. 2005. High-frequency conjugation system facilitates biofilm formation and pAM beta 1 transmission by Lactococcus lactis. Appl Environ Microb 71:2970-2978.

50. Maddocks, S. E., C. J. Wright, A. H. Nobbs, J. L. Brittan, L. Franklin, N. Stromberg, A. Kadioglu, M. A. Jepson, and H. F. Jenkinson. 2011. Streptococcus pyogenes antigen I/IIfamily polypeptide AspA shows differential ligand-binding properties and mediates biofilm formation. Mol Microbiol 81:1034-1049.

51. Maeyama, R., Y. Mizunoe, J. M. Anderson, M. Tanaka, and T. Matsuda. 2004. Confocal imaging of biofilm formation process using fluoroprobed Escherichia coli and fluorostained exopolysaccharide. J Biomed Mater Res A 70:274-282.

52. Manetti, A., C. Zingaretti, F. Falugi, S. Capo, M. Bombaci, F. Bagnoli, G. Gambellini, G. Bensi, M. Mora, A. Edwards, J. Musser, E. Graviss, J. Telford, G. Grandi, and I. Margarit. 2007. Streptococcus pyogenes pili promote pharyngeal cell adhesion and biofilm formation. Mol Microbiol 64:968-983.

53. Musser, J. M., V. Kapur, J. Szeto, X. Pan, D. S. Swanson, and D. R. Martin. 1995. Genetic diversity and relationships among Streptococcus pyogenes strains expressing serotype M1 protein: recent intercontinental spread of a subclone causing episodes of invasive disease. Infect Immun 63:994-1003.

54. Nadell, C. D., J. B. Xavier, and K. R. Foster. 2009. The sociobiology of biofilms. FEMS Microbiol. Rev. 33:206-224.

55. Pahlman, L. I., P. F. Marx, M. Morgelin, S. Lukomski, J. C. M. Meijers, and H. Herwald. 2007. Thrombin-activatable fibrinolysis inhibitor binds to Streptococcus pyogenes by interacting with collagen-like proteins A and B. J Biol Chem. 282:24873-24881.

56. Pan, W. H., P. L. Li, and Z. Liu. 2006. The correlation between surface hydrophobicity and adherence of Bifidobacterium strains from centenarians' faeces. Anaerobe 12:148152.

57. Piard, J. C., I. Hautefort, V. A. Fischetti, S. D. Ehrlich, M. Fons, and A. Gruss. 1997. Cell wall anchoring of the Streptococcus pyogenes M6 protein in various lactic acid bacteria. J Bacteriol 179:3068-3072.

58. Piard, J. C., R. JimenezDiaz, V. A. Fischetti, S. D. Ehrlich, and A. Gruss. 1997. The M6 protein of Streptococcus pyogenes and its potential as a tool to anchor biologically active molecules at the surface of lactic acid, bacteria. Streptococci and the Host 418:545-550.

59. Que, Y. A., J. A. Haefliger, P. Francioli, and P. Moreillon. 2000. Expression of Staphylococcus aureus clumping factor A in Lactococcus lactis subsp. cremoris using a new shuttle vector. Infect Immun 68:3516-3522.

60. Rasmussen, M., A. Eden, and L. Björck. 2000. SclA, a novel collagen-like surface protein of Streptococcus pyogenes. Infect Immun 68:6370-6377.

61. Reuter, M., C. C. Caswell, S. Lukomski, and P. F. Zipfel. 2010. Binding of the human complement regulators CFHR1 and factor $\mathrm{H}$ by streptococcal collagen-like protein 1 (Scl1) via their conserved $\mathrm{C}$ termini allows control of the complement cascade at multiple levels. J Biol Chem 285:38473-38485. 
62. Roberts, A. L., K. L. Connolly, C. D. Doern, R. C. Holder, and S. D. Reid. 2010. Loss of the group A Streptococcus regulator Srv decreases biofilm formation in vivo in an otitis media model of infection. Infect Immun 78:4800-4808.

63. Sharkawy, A., D. E. Low, R. Saginur, D. Gregson, B. Schwartz, P. Jessamine, K. Green, and A. McGeer. 2002. Severe group a streptococcal soft-tissue infections in Ontario: 1992-1996. Clin Infect Dis 34:454-460.

64. Silva, G. T., S. Chen, A. C. Frery, J. F. Greenleaf, and M. Fatemi. 2005. Stress field forming of sector array transducers for vibro-acoustography. IEEE Trans Ultrason Ferroelectr Freq Control 52:1943-1951.

65. Top, F. H., Jr., L. W. Wannamaker, W. R. Maxted, and B. F. Anthony. 1967. M antigens among group A streptococci isolated from skin lesions. J Exp Med 126:667-685.

66. Wadstrom, T., K. H. Schmidt, O. Kuhnemund, J. Havlicek, and W. Kohler. 1984. Comparative studies on surface hydrophobicity of streptococcal strains of group-A, group-B, group-C, group-D and group-G. J Gen Microbiol 130:657-664.

67. Whatmore, A. M., V. Kapur, D. J. Sullivan, J. M. Musser, and M. A. Kehoe. 1994. Noncongruent relationships between variation in $\mathrm{emm}$ gene sequences and the population genetic structure of group A streptococci. Mol Microbiol 14:619-631.

68. Xu, Y., D. R. Keene, J. M. Bujnicki, M. Höök, and S. Lukomski. 2002. Streptococcal Scl1 and Scl2 proteins form collagen-like triple helices. J Biol Chem 277:27312-27318. 


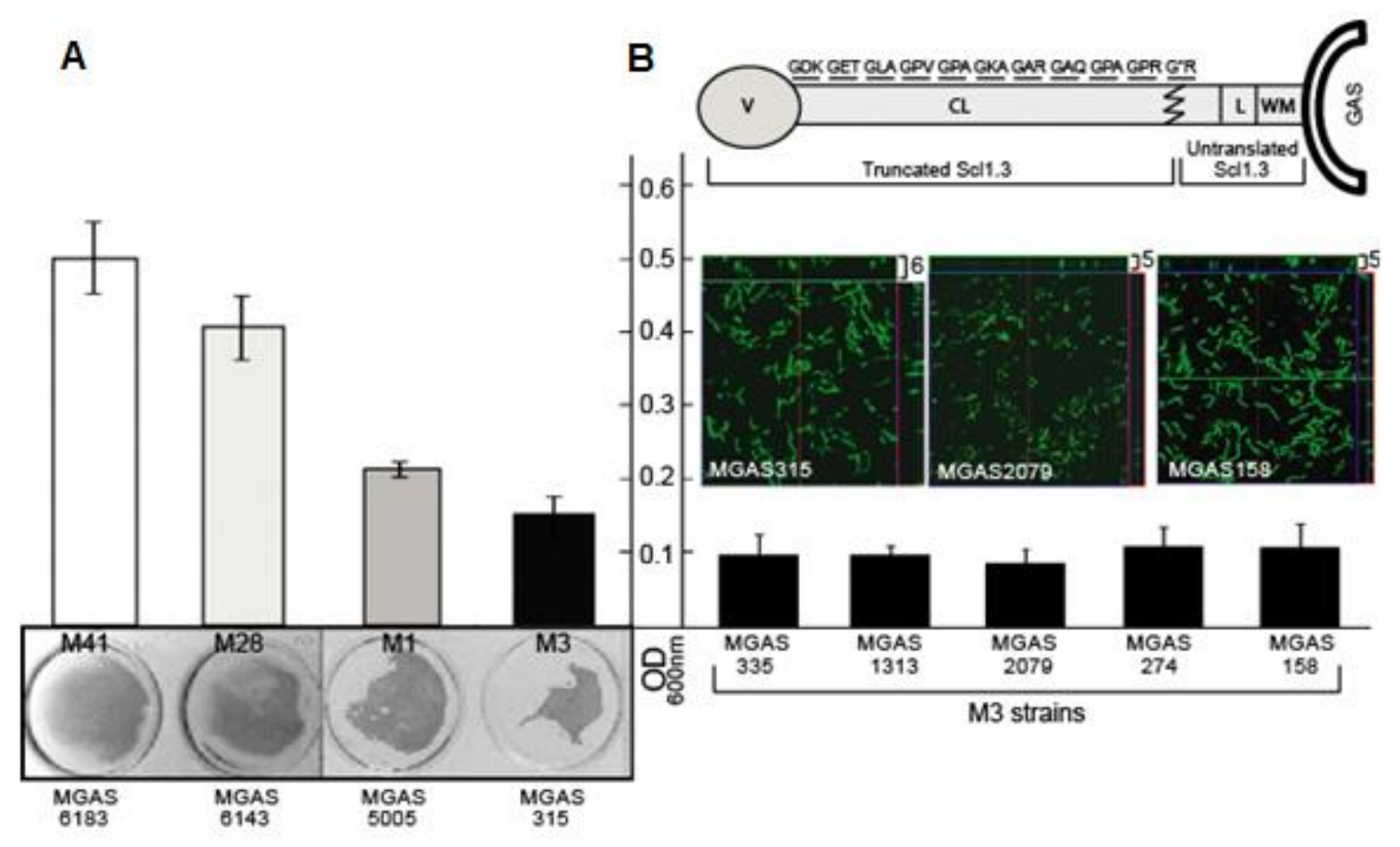

Figure 1. Variation in biofilm formation among GAS strains.

(A) Wild type M41-, M28-, M3-, and M1-type GAS strains were grown $24 \mathrm{~h}$ under static conditions and analyzed spectrophotometrically following crystal violet staining (top). Visual representation of corresponding wells is shown below. (B) Schematic representation (not to scale) of Scl1.3 protein of M3-type GAS. Translated $G X Y$ repeats within the collagen-like $(C L)$ region are shown with an asterisk representing the location of the premature stop codon resulting in a truncated protein. V, variable region; L, linker region; WM, wall-membrane associated region. Below, spectrophotometric measurements of 24-h biofilms following crystal violet staining are graphed for M3-type GAS strains. Absorbance values $\left(\mathrm{OD}_{600 \mathrm{~nm}}\right)$ are averages of at least three experiments done in triplicate wells. Corresponding confocal analyses of 24-h biofilms of MGAS315, MGAS2079, and MGAS158 are shown. Images are X-Y orthogonal Z-stack views and average vertical thickness is indicated in micrometers (top right). 
A

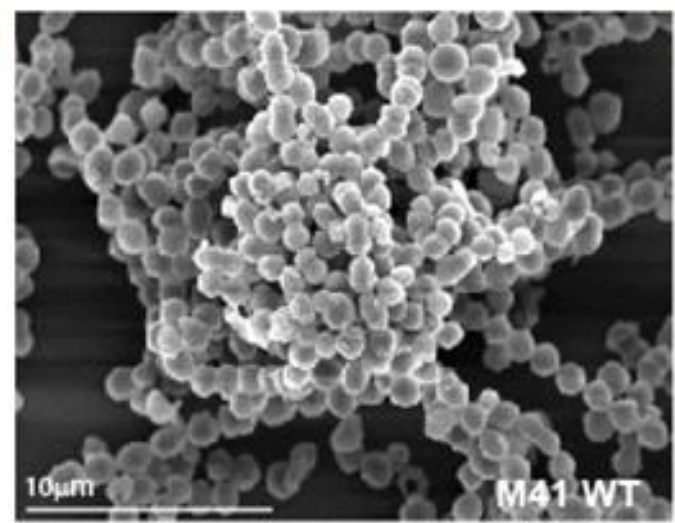

C

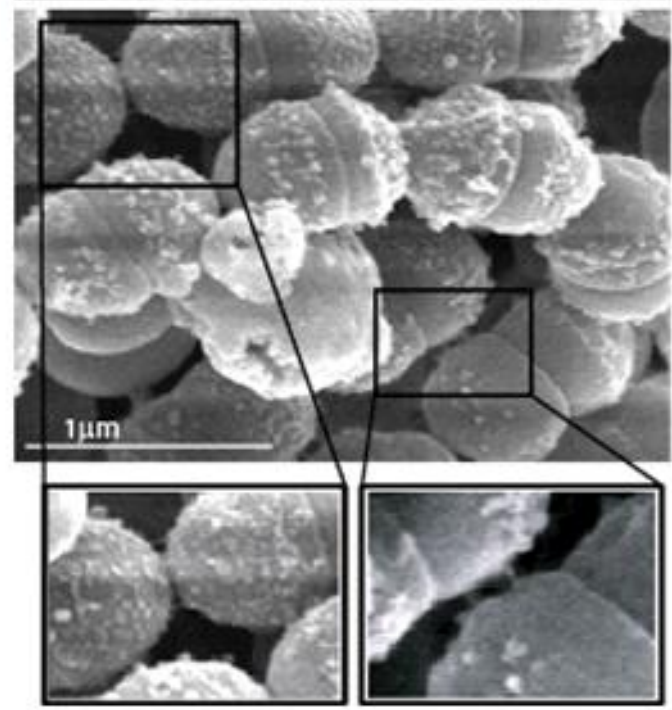

B

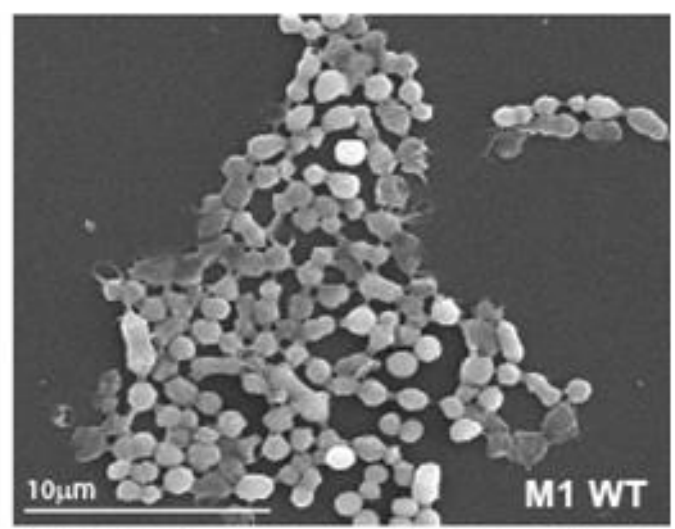

D

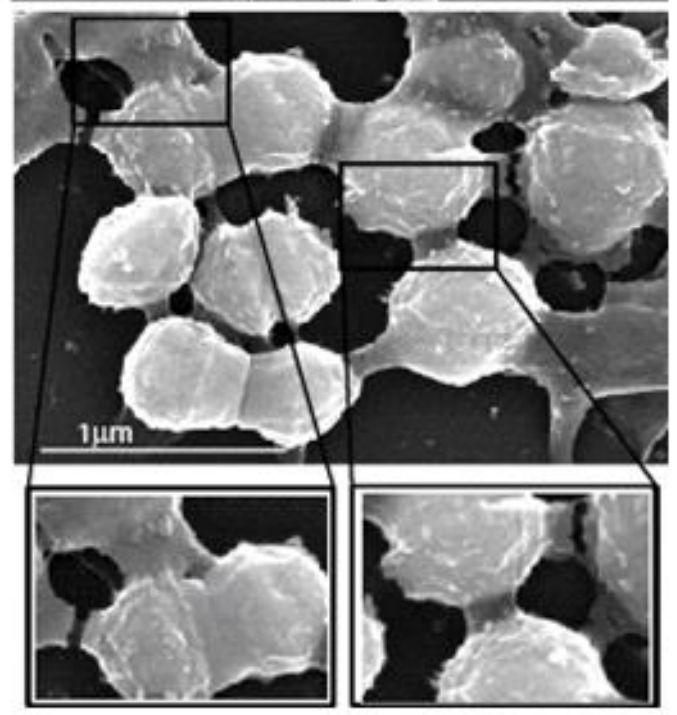

Figure 2. Field emission scanning electron microscopy of GAS biofilms. 24$\mathrm{h}$ biofilms of the M1- and M41-type GAS strains were grown on glass cover slips and analyzed by FESEM. (A-B) Architecture of GAS microcolonies shown at low magnification. (C-D) Cell surface morphology and cell-to-cell junctions observed at higher magnification. Enlargements of cell-to-cell junctions are shown below. 

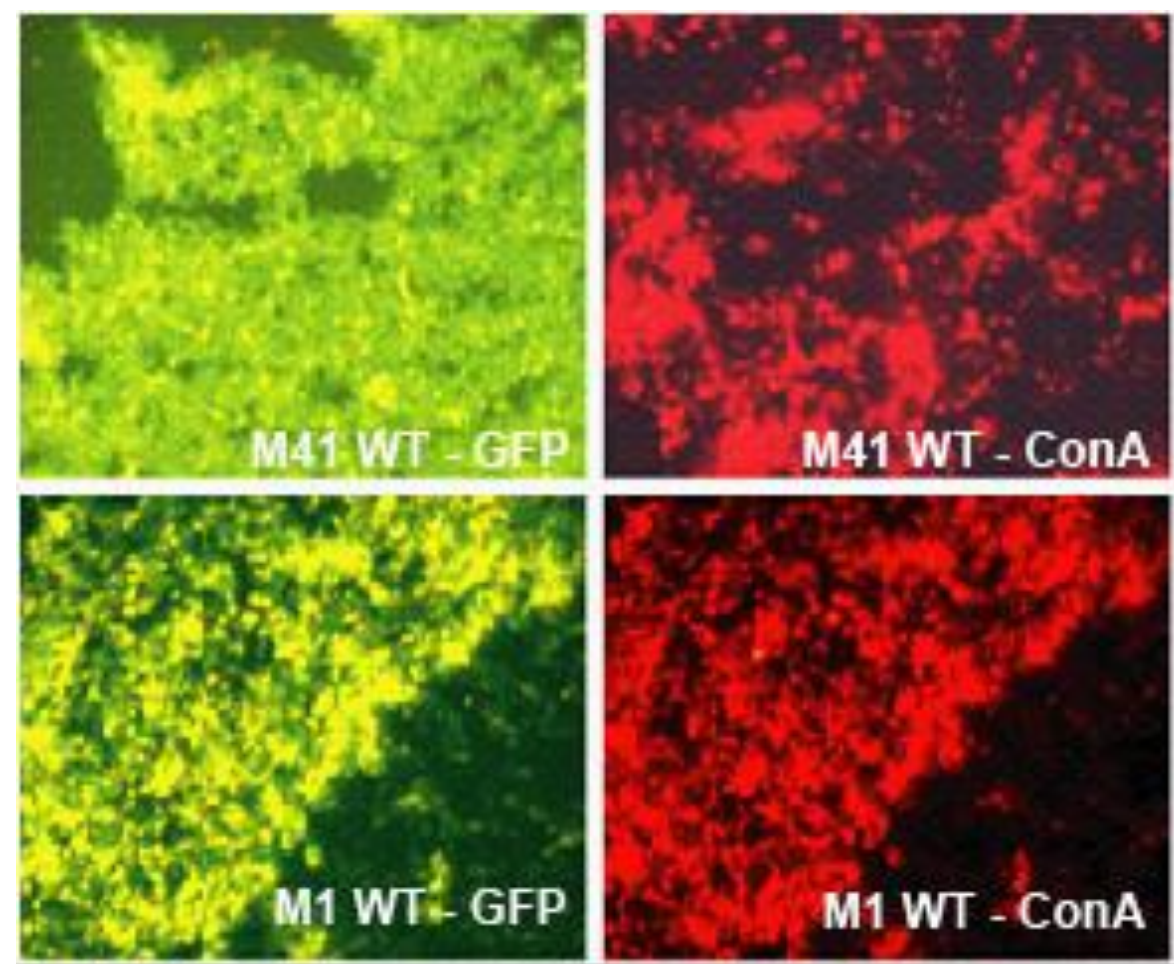

Figure 3. Production of bacterial-associated extracellular matrix. GFPexpressing wild type (WT) M41- and M1-type GAS strains were grown on glass cover slips for $24 \mathrm{~h}$ and stained with TRITC-conjugated concanavalin A (ConA). Confocal laser scanning microscopic (CLSM) images were separated to represent green GFP-expressing GAS cells (left images) and red ConA-TRITC staining (right images) for detection of extracellular matrix associated with each strain. Images are from one representative experiment of three. 


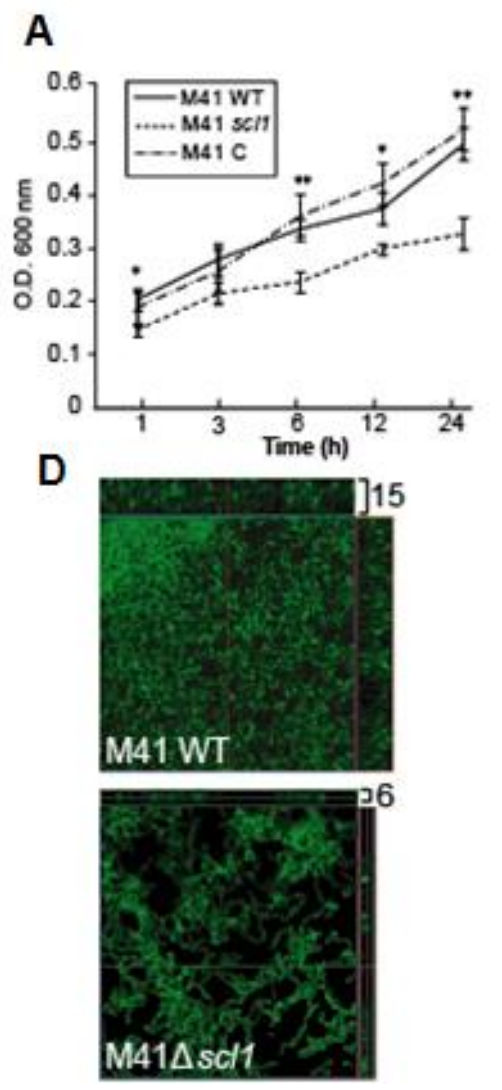

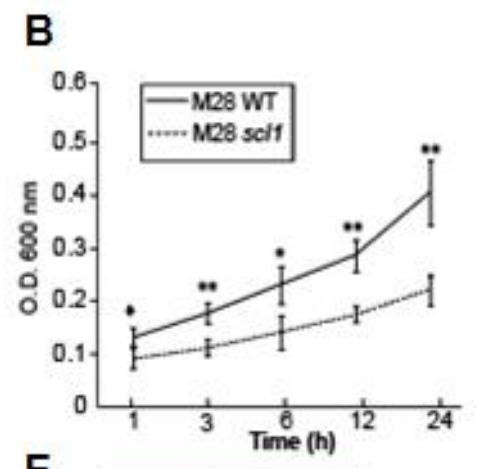

E
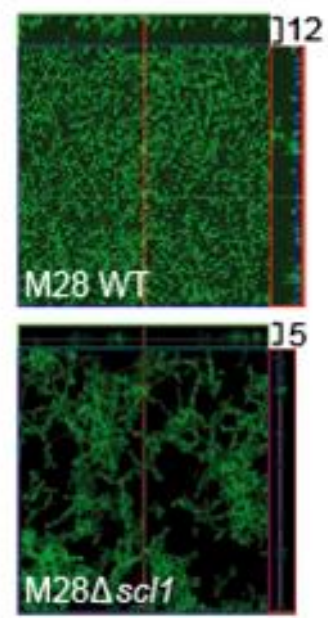

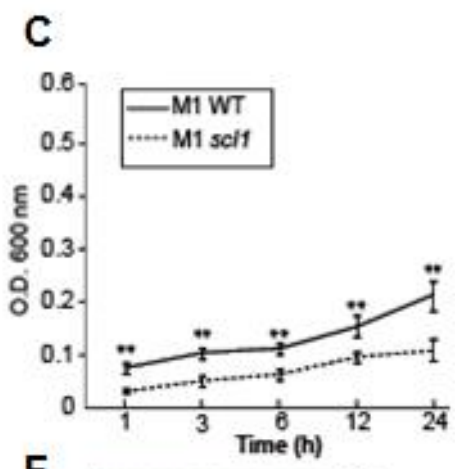

$\mathbf{F}$
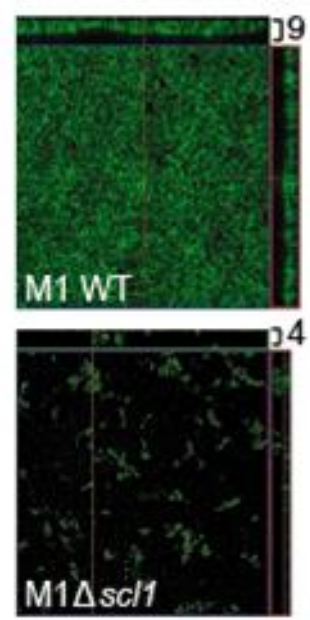

Figure 4. Biofilm formation by wild type and sc/1-inactivated isogenic mutants. Crystal violet staining and confocal laser scanning microscopy (CLSM) of the GFP-expressing GAS were used to compare biofilm formation by GAS strains. Wild type (WT) M41-, M28-, and M1-type strains, sc/1-inactivated mutants (sc/1), and M41 mutant complemented for Scl1.41 expression (M41 C) were used. (A-C) Isogenic GAS strains were grown under static conditions for 24 $\mathrm{h}$ and bacterial biomass was detected spectrophotometrically at indicated time points following crystal violet staining. Absorbance values at $\mathrm{OD}_{600 \mathrm{~nm}}$ are representative of at least three experiments performed in quadruplicate. Statistical significance is denoted as ${ }^{*} P \leq 0.05$ and ${ }^{* *} P \leq 0.001$. (D-F) CLSM analysis of corresponding $24 \mathrm{~h}$ biofilms from same experiment. Images are $\mathrm{X}-\mathrm{Y}$ orthogonal Z-stack views of WT (top) and mutant (bottom) GAS strains. Views are representative of ten images within a single experiment. Average vertical biofilm thickness is indicated in micrometers (top right). 


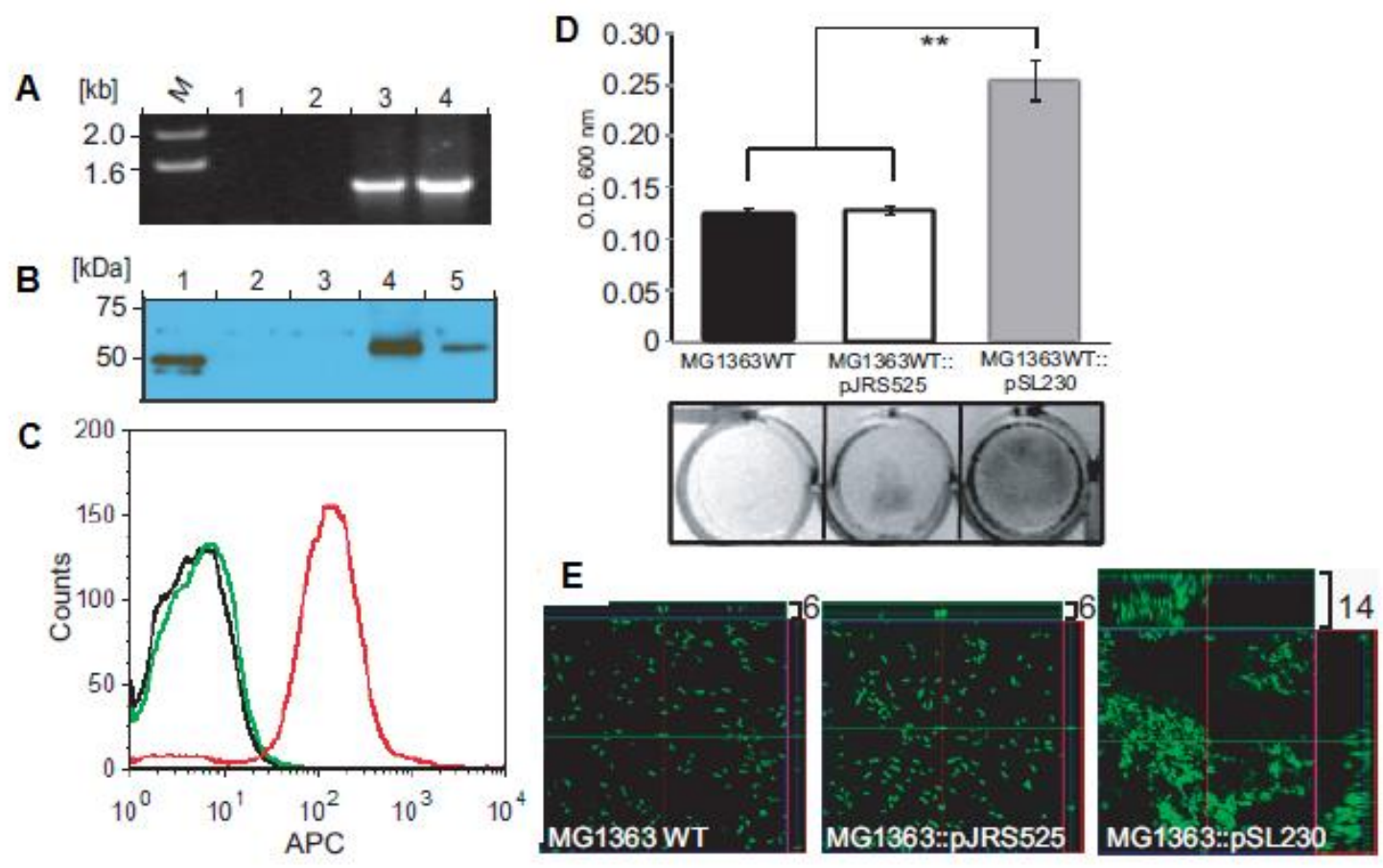

Figure 5. Scl1 expression in L. lactis promotes biofilm formation. L. lactis was transformed with the plasmid construct pSL230 to express Scl1.41 surface protein or with pJRS525 vector. (A) PCR analysis of L. lactis transformants using scl1.41-gene-specific primers; lanes: (1) MG1363 wild-type (WT) cells; (2) MG1363::pJRS525 vector-only control; (3) MG1363::pSL230 transformant; (4) control pSL230 plasmid DNA. (B) Scl1.41 expression by western blot analysis of cell-wall extracts prepared from transformed L. lactis and control GAS strains using anti- P176 (rScl1.41) antibodies; lanes: (1) purified recombinant P176 protein (truncated Scl1.41); (2) MG1363 WT strain; (3) MG1363::pJRS525 vector; (4) MG1363::pSL230 transformant; (5) MGAS6183 (M41) control. (C) Analysis of Scl1.41 expression by flow cytometry with anti-P176 (rScl1.41) rabbit polyclonal antibodies on the surface of MGAS1363 WT strain (black trace), MGAS1363::pJRS525 vector-only control (green trace) and MG1363::pSL230 transformant (red trace). (D) Crystal violet staining of $24 \mathrm{~h}$ biofilms formed by $L$. lactis WT strain, MG1363::pJRS525 vector-only control or MG1363::pSL230 transformant (top) with visual representation of the corresponding wells (bottom). Statistical significance is denoted as ${ }^{* *} P \leq 0.001$. (E) CLSM analysis of $24 \mathrm{~h}$ biofilms from same experiment shown in (D). Images are X-Y orthogonal Z-stack views representative of ten images within a single experiment. Average vertical biofilm thickness is indicated in micrometers (top right). 


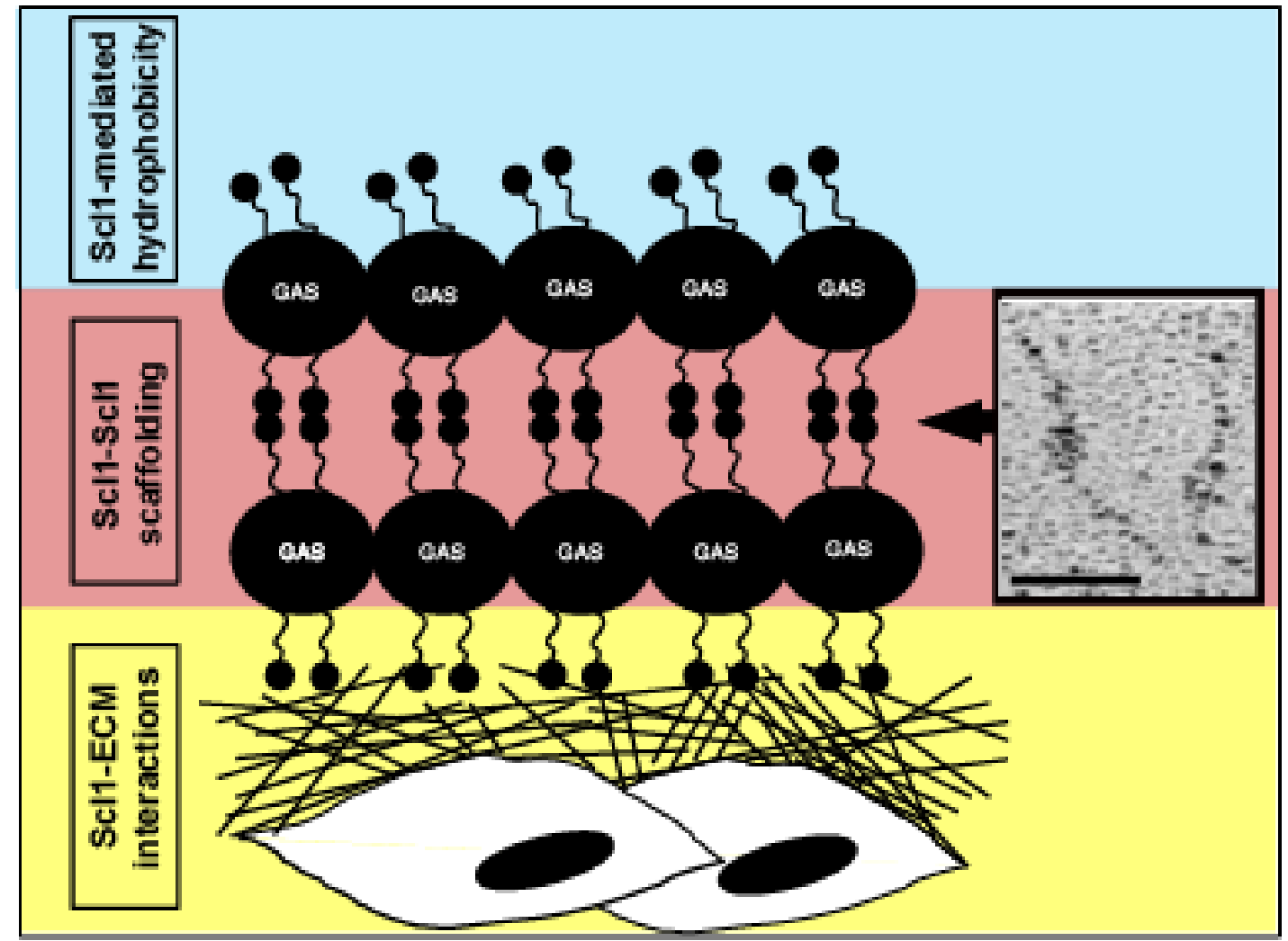

Figure 6- Scl1-mediated model of GAS biofilm (not to scale). Scl1 hydrophobin (current study) initiates bacterial adhesion to animate surfaces (21) within the host (blue field). Scl1 adhesin anchors the growing microcolony by direct binding to tissue extracellular matrix (ECM) components, cellular fibronectin and laminin (17), initiating microcolony formation and anchoring the outside edge of GAS microcolony in tissue (yellow field). Microcolony scaffolding is stabilized by the formation of head-to-head dimers between Scl1 molecules on adjacent chains (pink field). Inset shows Scl1-Scl1 head-to-head dimers formed by rScl1.1 as viewed by electron microscopy after rotary shadowing (68). Bar. 50 $\mathrm{nm}$. 


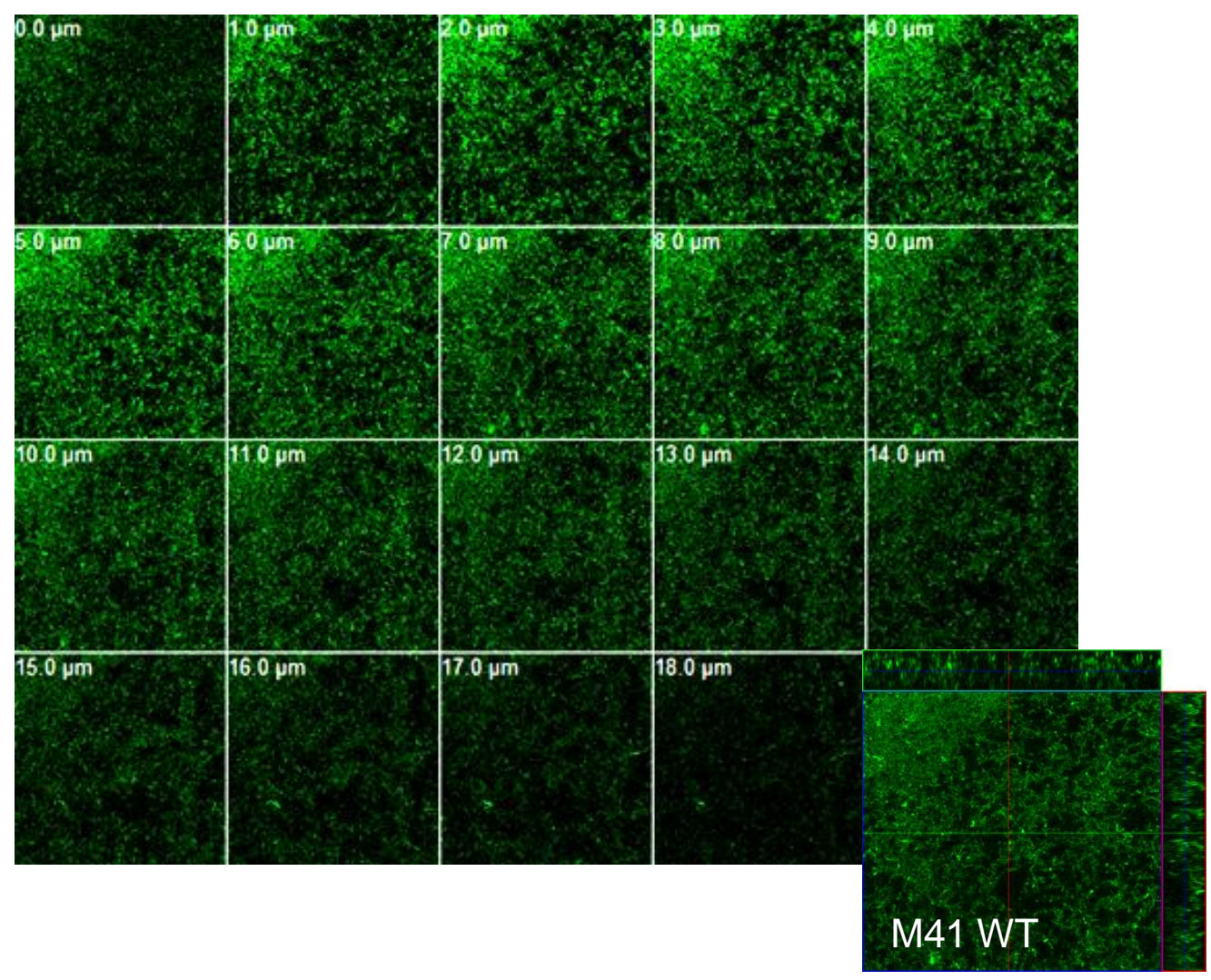

Figure S1 (a). Confocal laser scanning microscopy (CLSM) of GFP-expressing M41 wild type (WT) GAS biofilm at $24 \mathrm{~h}$. Panels represent a gallery view of consecutive images taken at 1 micrometer increments. Panel shown in lower right corner represents an X-Y orthogonal Z-stack view (Fig. 4d). Thickness is indicated in micrometers 


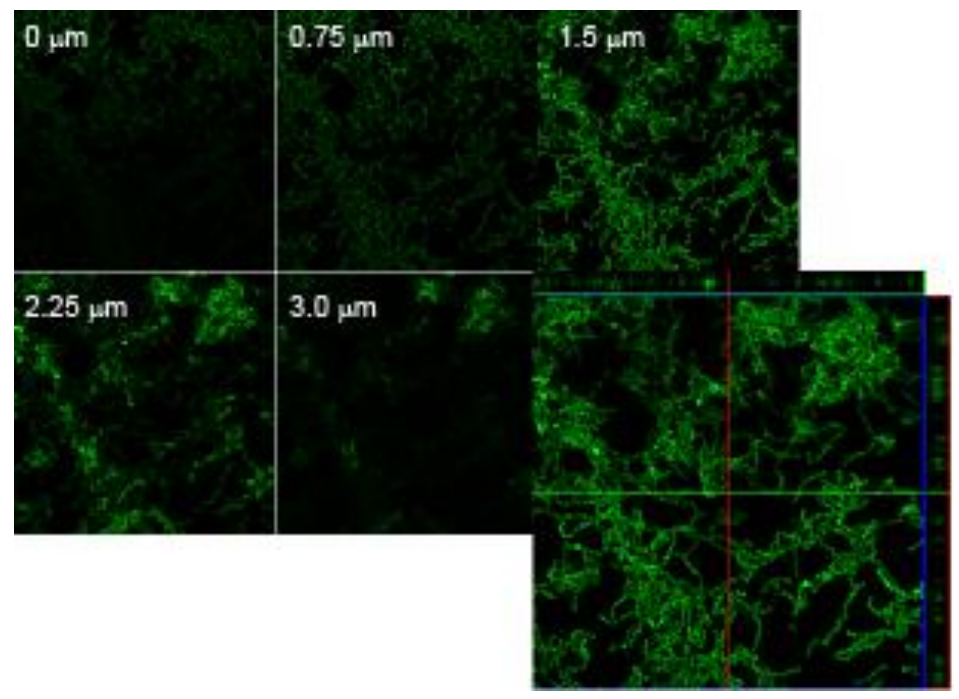

Figure S1 (b). Confocal laser scanning microscopy (CLSM) of GFP-expressing M41 $\Delta s c / 1$ GAS biofilm at $24 \mathrm{~h}$. Panels represent a gallery view of consecutive images taken at 0.75 micrometer increments. Panel shown in lower right corner represents an X-Y orthogonal Z-stack view (Fig. 4d). Thickness is indicated in micrometers. 


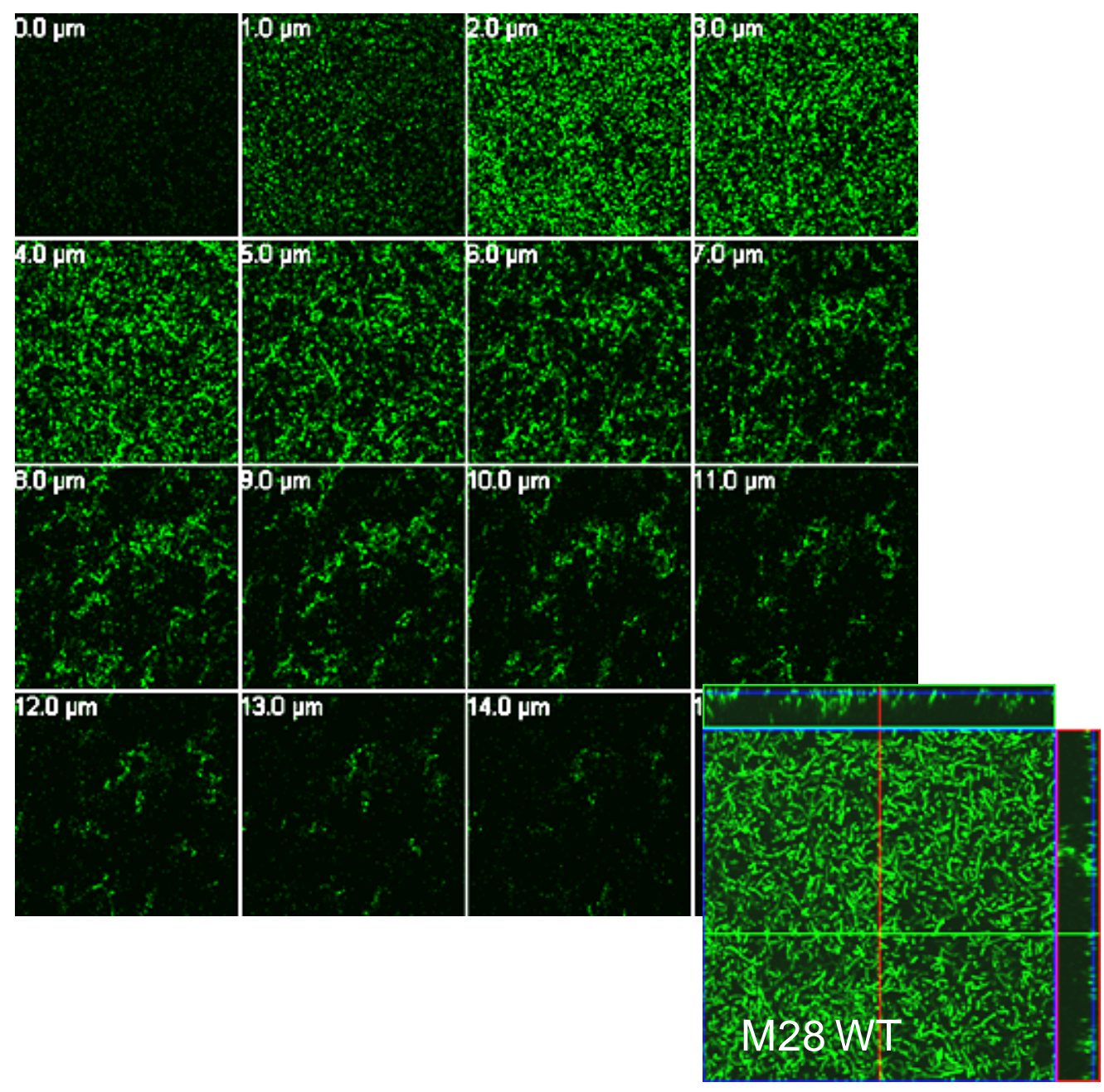

Figure S1 (c). Confocal laser scanning microscopy (CLSM) of GFP-expressing M28 WT GAS biofilm at $24 \mathrm{~h}$. Panels represent a gallery view of consecutive images taken at 1 micrometer increments. Panel shown in lower right corner represents an $X-Y$ orthogonal Z-stack view (Fig. 4e). Thickness is indicated in micrometers. 


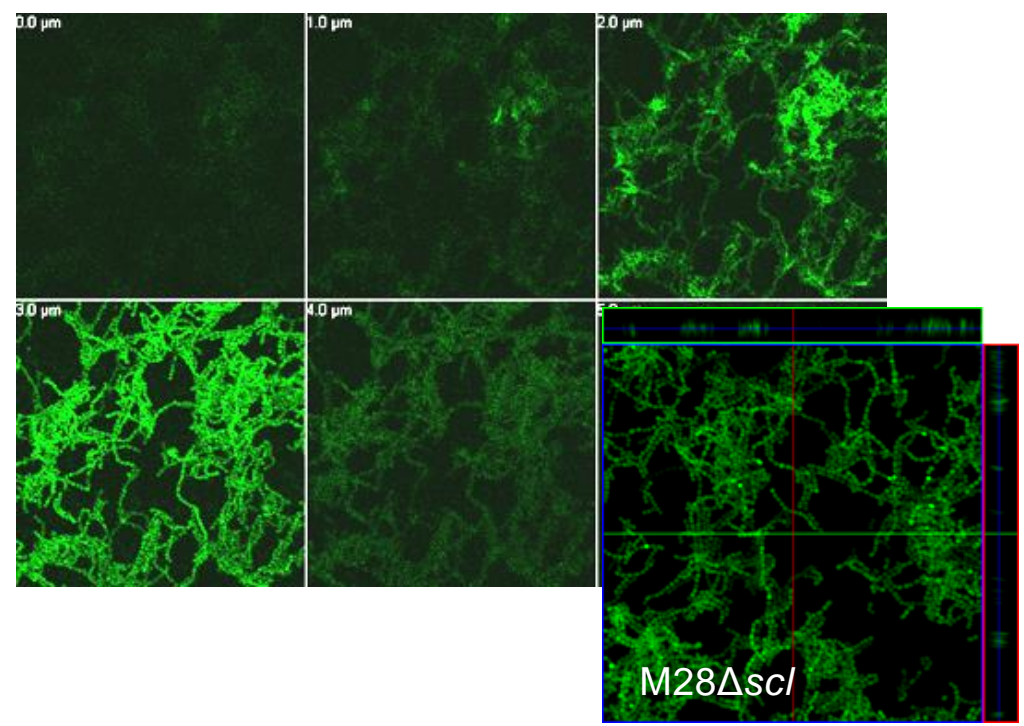

Figure S1 (d). Confocal laser scanning microscopy (CLSM) of GFP-expressing $\mathrm{M} 28 \Delta s c / 1 \mathrm{GAS}$ biofilm at $24 \mathrm{~h}$. Panels represent a gallery view of consecutive images taken at 1 micrometer increments. Panel shown in lower right corner represents an X-Y orthogonal Z-stack view (Fig. 4e). Thickness is indicated in micrometers. 


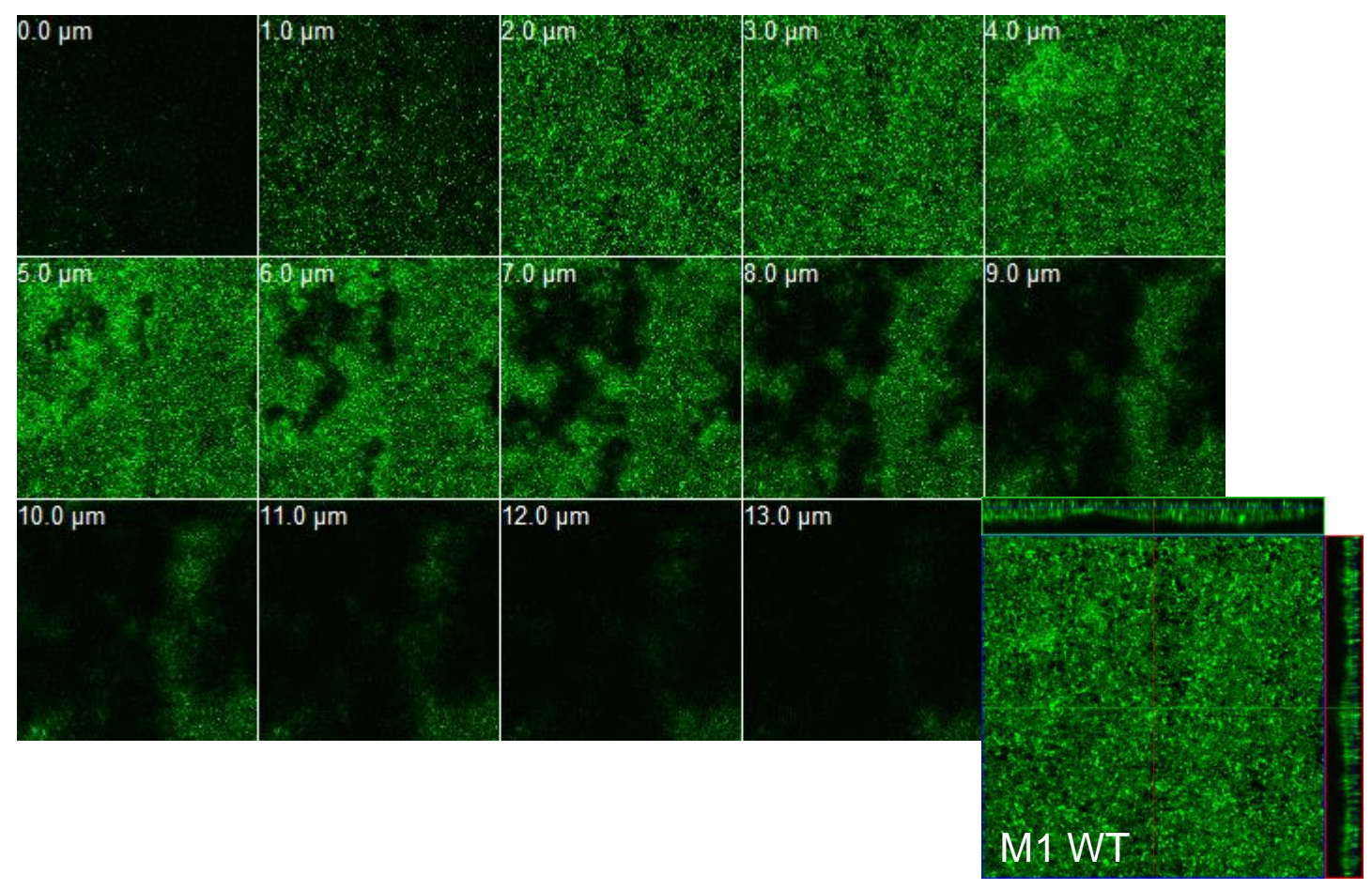

Figure S1 (e). Confocal laser scanning microscopy (CLSM) of GFP-expressing M1 wild type (WT) GAS biofilm at $24 \mathrm{~h}$. Panels represent a gallery view of consecutive images taken at 1 micrometer increments. Panel shown in lower right corner represents an $X-Y$ orthogonal Z-stack view (Fig. 4f). Thickness is indicated in micrometers. 


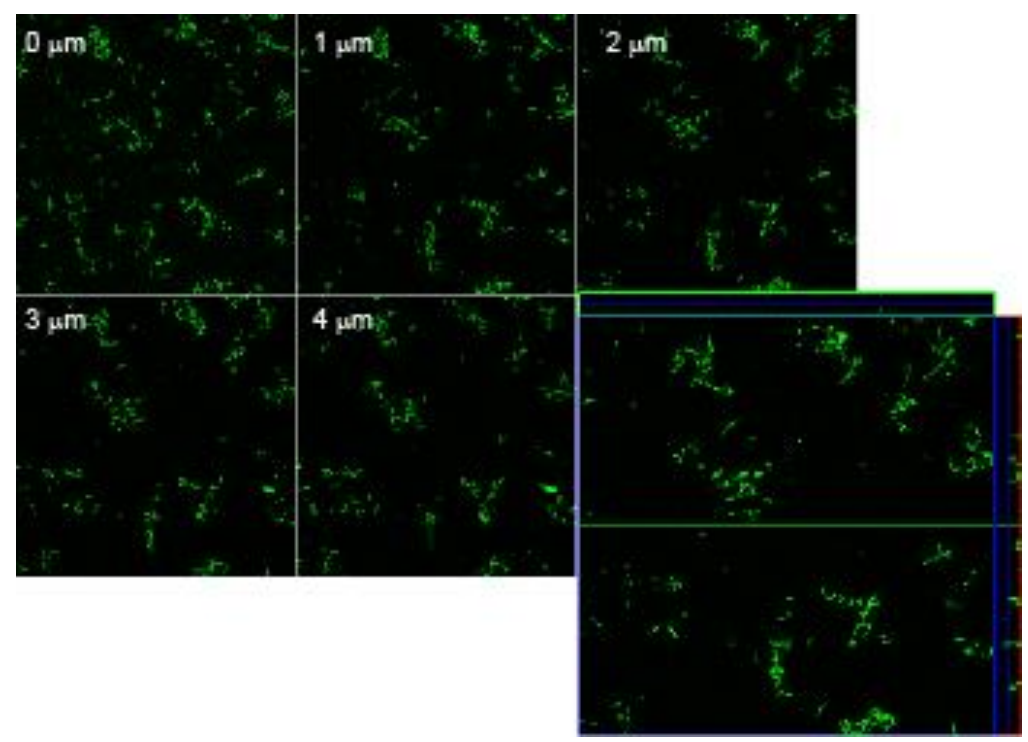

Figure S1 (d). Confocal laser scanning microscopy (CLSM) of GFP-expressing $\mathrm{M} 1 \Delta s c / 1 \mathrm{GAS}$ biofilm at $24 \mathrm{~h}$. Panels represent a gallery view of consecutive images taken at 1 micrometer increments. Panel shown in lower right corner represents an X-Y orthogonal Z-stack view (Fig. 4f). Thickness is indicated in micrometers. 


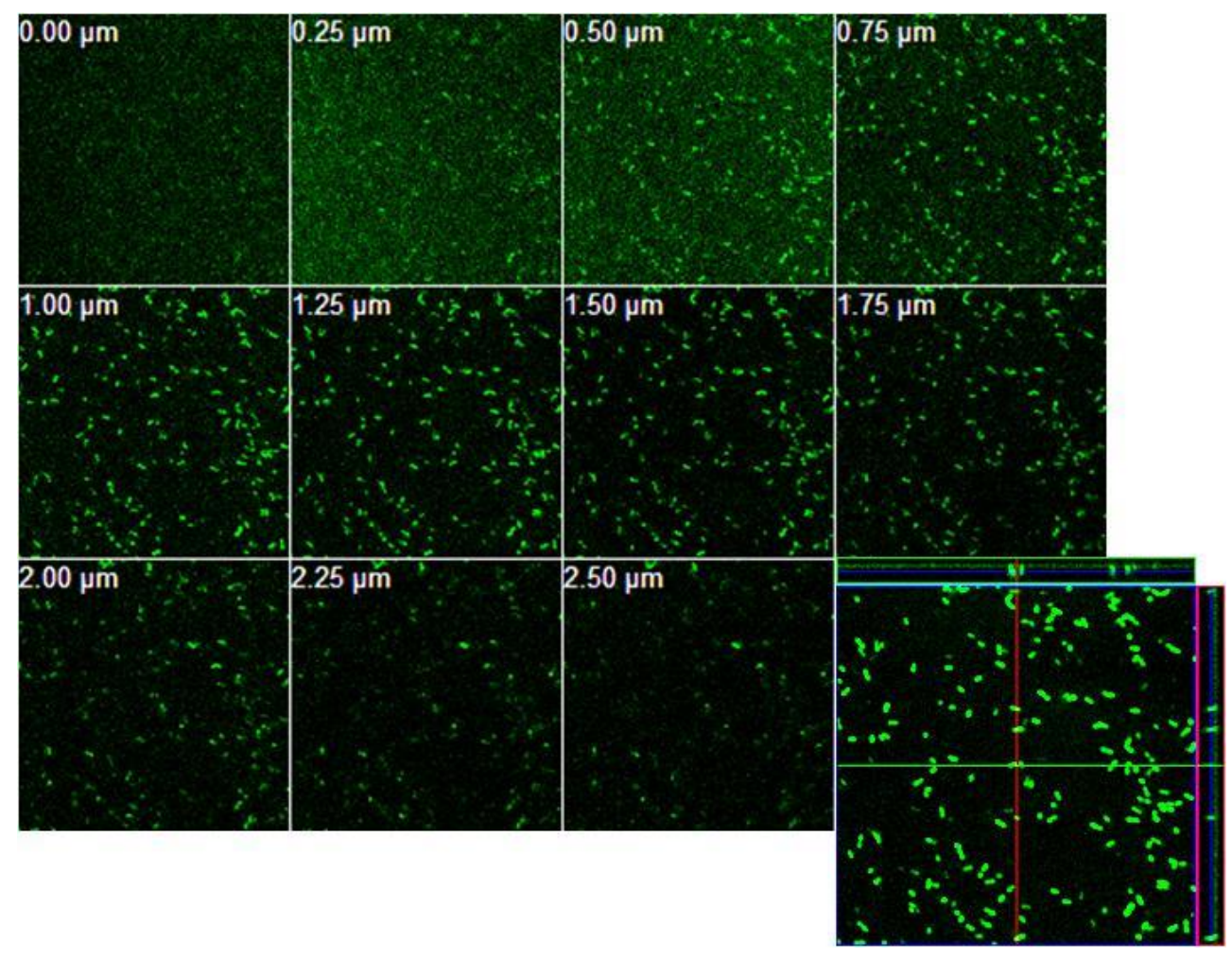

Figure S2 (a). Confocal laser scanning microscopy (CLSM) of GFP-expressing Lactococcus lactis MG1363 wild type (WT) biofilm at $24 \mathrm{~h}$. Panels represent a gallery view of consecutive images taken at 0.25 micrometer increments. Panel shown in lower right corner represents an X-Y orthogonal Z-stack view (5e). Thickness is indicated in micrometers. 


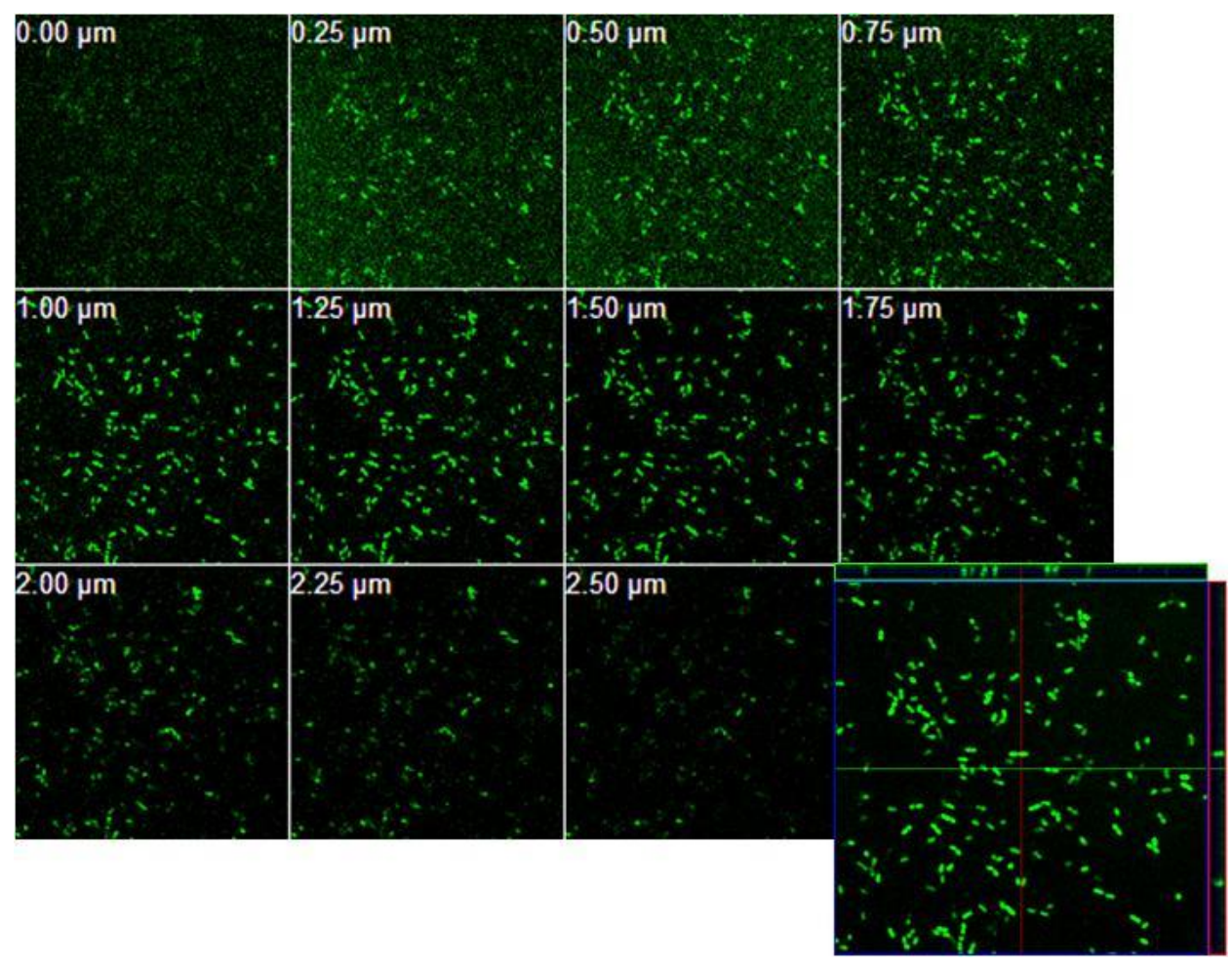

Figure S2 (b). Confocal laser scanning microscopy (CLSM) of GFP-expressing Lactococcus lactis biofilm at $24 \mathrm{~h}$. L. lactis was transformed with the shuttle vector pJRS525 (MG1363::pJRS525). Panels represent a gallery view of consecutive images taken at 0.25 micrometer increments. Panel shown in lower right corner represents an $\mathrm{X}-\mathrm{Y}$ orthogonal Z-stack view (5e). Thickness is indicated in micrometers. 


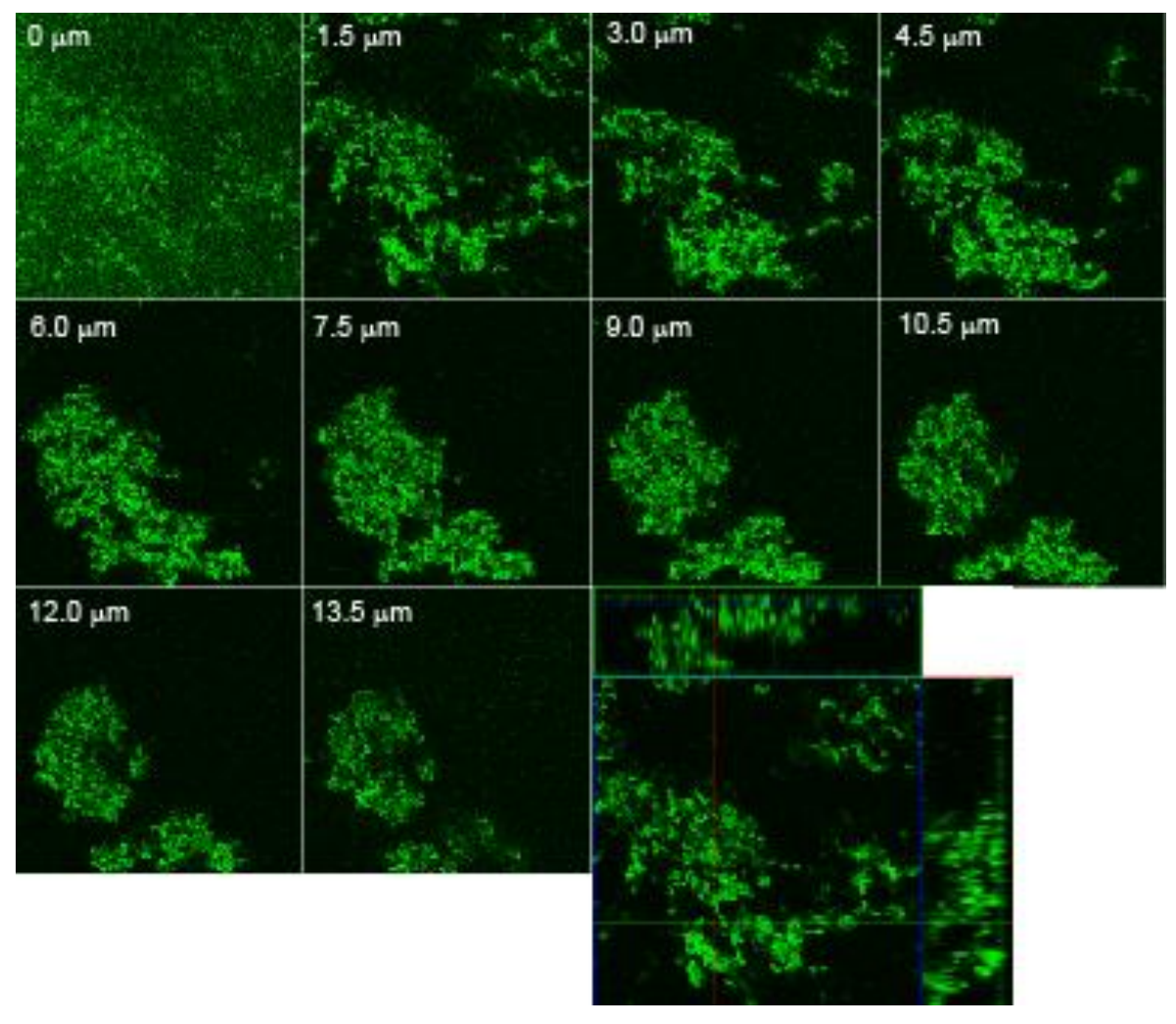

Figure S2 (c). Confocal laser scanning microscopy (CLSM) of GFP-expressing Lactococcus lactis expressing Scl1.41 protein (MG1363::pSL230) biofilm at $24 \mathrm{~h}$. L. lactis was transformed with the plasmid construct pSL230 encoding Scl1.41 protein (MG1363::pSL230). Panels represent a gallery view of consecutive images taken at 0.5 micrometer increments. Panel shown in lower right corner represents an $X-Y$ orthogonal Z-stack view (5e). Thickness is indicated in micrometers. 
Table 1. Cell surface hydrophobicity of GAS strains

\begin{tabular}{cccc} 
GAS Strain & M-Type & Actual Value $^{\dagger}$ & Hydrophobicity Index $^{\ddagger}$ \\
\hline MGAS6183 WT & M41 & $92.6 \pm .86$ & 100 \\
MGAS6183 $\Delta s c / 1$ & M41 & $85.2 \pm 2.2$ & ${ }^{* * 92}$ \\
MGAS6183 $\Delta s c / 1-C$ & M41 & $98.0 \pm .31$ & 105 \\
\hline MGAS5005 WT & M1 & $80.3 \pm .89$ & 100 \\
MGAS5005 $\Delta s c / 1$ & M1 & $63.3 \pm 3.2$ & $* 79$ \\
\hline MGAS6143 WT & M28 & $94.3 \pm .73$ & 100 \\
MGAS6143 $\Delta s c / 1$ & M28 & $72.6 \pm .62$ & $* 78$ \\
\hline
\end{tabular}

$\dagger^{\dagger}$ Actual hydrophobicity values were calculated based on hexadecane binding as described in Methods. Values are representative of three separate experiments with ten replicates $\pm S D$.

$\mp$ Hydrophobicity Index represents the ration of actual hydrophobicity value for each strain to that of the isogenic wild-type (WT) strain multiplied by 100.

${ }^{* *}$ Asterisks denote a statistically significant difference of $\Delta s c / 1$ mutants versus WTs at $P \leq 0.001$. 
Table 2. Cell surface hydrophobicity of Lactococcus strains

Lactococcus Strain

Actual Value $^{\dagger} \quad$ Hydrophobicity Index ${ }^{\ddagger}$

L.lactis 1363 WT

$59.7 \pm 7.2$

100

L. lactis 1363::pJRS525

$56.6 \pm 5.5$

98

L. lactis 1363::pSL230

$82.0 \pm 2.6$

$\star * 137$

${ }^{\dagger}$ Actual hydrophobicity values were calculated based on hexadecane binding as described in Methods. Values are representative of three separate experiments with ten replicates $\pm S D$.

$\mp$ Hydrophobicity Index represents the ration of actual hydrophobicity value for each strain to that of the isogenic wild-type (WT) strain multiplied by 100.

${ }^{* *}$ Asterisks denote a statistically significant difference of $\Delta s c / 1$ mutants versus WTs at $P \leq 0.001$. 
Chapter 4:

Streptococcal Collagen-like Protein-1 (Scl1) Targets a Specific Variant of Cellular Fibronectin Expressed in Wounded Tissue 
The group A streptococcal collagen-like protein 1, Scl1, mediates biofilm formation by targeting the EDA-containing variant of cellular fibronectin expressed in wounded tissue

\section{Heaven Oliver-Kozup, ${ }^{1}$ Karen H. Martin, ${ }^{2,3}$ Diane Schwegler-Berry, ${ }^{4}$ Brett J. Green, ${ }^{5}$ Courtney Betts, ${ }^{6 \dagger}$ Arti V. Shinde, ${ }^{6 \ddagger}$ Livingston Van De Water, ${ }^{6}$ and Slawomir Lukomski ${ }^{1^{*}}$}

${ }^{1}$ Department of Microbiology, Immunology, and Cell Biology; ${ }^{2}$ Mary Babb Randolph Cancer Center; and ${ }^{3}$ Microscope Imaging Facility; West Virginia University School of Medicine, Morgantown, WV 26506, USA.

${ }^{4}$ Pathology and Physiology Research Branch and ${ }^{5}$ Allergy and Clinical Immunology Branch, Health Effects Laboratory Division, National Institute of Occupational Safety and Health (NIOSH), Centers for Disease Control and Prevention, Morgantown, WV 26505, USA.

${ }^{6}$ Center for Cell Biology and Cancer Research, Albany Medical College. Albany, NY 12208, USA.

Published in Molecular Microbiology (2013) 87[3], 672-689 


\section{Summary}

Wounds are known to serve as portals of entry for group A Streptococcus (GAS). Subsequent tissue colonization is mediated by interactions between GAS surface proteins and host extracellular matrix components. We recently reported that the streptococcal collagen-like protein-1, Scl1, selectively binds the cellular form of fibronectin (cFn) and also contributes to GAS biofilm formation on abiotic surfaces. One structural feature of cFn, which is predominantly expressed in response to tissue injury, is the presence of a spliced variant containing extra domain $A(E D A / E I I I A)$. We now report that GAS biofilm formation is mediated by the Scl1 interaction with EDA-containing cFn. Recombinant Scl1 proteins that bound $\mathrm{cFn}$ also bound recombinant EDA within the C-C' loop region recognized by the $\alpha_{9} \beta_{1}$ integrin. The extracellular 2-D matrix derived from human dermal fibroblasts supports GAS adherence and biofilm formation. Altogether, this work identifies and characterizes a novel molecular mechanism by which GAS utilizes Scl1 to specifically target an extracellular matrix component that is predominantly expressed at the site of injury in order to secure host tissue colonization. 
Group A Streptococcus (GAS) is responsible each year for more than 730 million infections world-wide ranging from the clinically uncomplicated (pharyngitis, impetigo) to severe and invasive diseases (necrotizing fasciitis, toxic shock), and autoimmune complications $(9,81)$. GAS causes over 100 million skin infections and is categorized as transient or contaminant flora of the skin (85). These infections are initiated via a portal of entry resulting from simple skin infringements, such as insect bites or cuts and scratches, as well as from more serious postsurgical wounds. For successful colonization within a wounded site, GAS utilizes surface adhesins to secure binding to host extracellular matrix (ECM) components (14), including collagen, fibronectin (Fn) and laminin.

The streptococcal collagen-like protein-1 (Scl1) is a ubiquitous surface adhesin, which is co-expressed with a range of known virulence factors that are regulated by the multiple virulence gene regulator of GAS (Mga) $(2,49,50,67)$. Scl1 is a homotrimeric protein protruding from the GAS surface that contains four structurally distinct regions. The outermost $\mathrm{N}$-terminal variable $(\mathrm{V})$ region is adjacent to a collagen-like $(\mathrm{CL})$ region that consists of a varying number of GlyXaaYaa (GXY) repeats and adopts stable collagen-like triple helices $(57,87)$. At the C-terminus, Scl1 contains a linker $(L)$ region which is a series of conserved, direct repeats adjoining the $\mathrm{CL}$ region to the cell wall/membrane (WM)-associated region. Functionally, Scl1 was shown to bind to host-cell integrin receptors $(10,11,36)$ and plasma components $(26,29,62)$. We recently reported that Scl1 selectively binds to cellular fibronectin (cFn), but not plasma 
fibronectin $(\mathrm{pFn})(13)$. Scl1 is also recognized to play a significant role in biofilm formation on abiotic surfaces (60).

The wound healing process is dependent on the interaction of cells with the surrounding ECM, which induces the formation of an ECM scaffold to promote cell migration and wound closure. A fundamental component within the ECM contributing to this process is cFn (56). The fibronectins form a large heterogeneous group of glycoproteins with isoforms resulting from alternative splicing events of pre-mRNA of a single gene (64). The soluble pFn is predominantly produced by hepatocytes, whereas cFn can be produced by diverse cell types and may generate up to 20 isoforms in humans. It contains varying amounts of two extra domains termed EDA (EIIIA) and EDB (EIIIB). EDA- and EDB-expressing cFn is highly regulated and plays a crucial role during embryogenesis and early development, and under non-diseased states is maintained at low levels within tissue of adult humans. The expression of cFn containing EDA (EDA/cFn) is upregulated during tissue repair and likely promotes wound cell function by the direct binding of $\alpha_{4} \beta_{1}, \alpha_{4} \beta_{7}$, and $\alpha_{9} \beta_{1}$ integrins $(25,35,42,46,71,77,84)$.

In this work, we characterized the Scl1-cFn interaction using recombinant Scl proteins ( $\mathrm{rScl}$ ) derived from epidemiologically distinct GAS strains and investigated the significance of this binding during GAS biofilm formation. We report that Scl1 protein selectively targets the EDA domain of $\mathrm{cFn}$, which is expressed during tissue repair. We mapped the Scl1 binding site within the C-C' loop region of EDA using peptide inhibition and antibody blocking assays, which 
overlaps with the EDA- $\alpha_{9} \beta_{1}$-integrin-binding site. Furthermore, the Scl1-ECM binding contributes significantly to GAS biofilm formation, which can be inhibited by either the treatment with the synthetic C-C' loop peptide or with the anti-EDA mAb, IST-9. In conclusion, this work identifies a novel cFn binding mechanism, which is conserved among pathogenically varying strains of GAS, and is mediated by Scl1 binding to the EDA/cFn variant that is expressed at the portal of pathogen entry. The Scl1-EDA/cFn binding may extend the time for GAS tissue colonization by impairing cFn-integrin binding and thereby delaying wound healing.

\section{Results}

The Scl1-cFn interaction is mediated by the EDA domain of $c F n$

In a previous study, we established that the Scl1 protein selectively binds the tissue extracellular matrix component, cFn (Fig. 1A) but not pFn (13). Based on this finding, we hypothesized that the Scl1-cFn interaction is mediated via EDA, a domain that is not found in plasma fibronectin, but is thought to be an integral component to the wound healing process. To test this hypothesis, we evaluated by direct ELISA the binding of ECM ligands to immobilized rScl1 proteins P176 (Scl1.41), P144 (Scl1.1), and P161 (Scl1.28) that were previously demonstrated to bind cFn (13). An immobilized rScl2 construct, P163 (Scl2.28), served as a negative control for cFn binding. The ECM ligands $(1 \mu \mathrm{g} /$ well) tested included: (i) purified cFn as a positive control, and confirmed to contain the EDA/cFn variant using the EDA-specific mAb IST-9, (ii) the rEDA, and (iii) the recombinant type III 
domain four (rlll4), which differs by primary sequence but is structurally homologous to EDA and is common to both $\mathrm{cFn}$ and pFn. As shown in Fig. 1B, rScl1 proteins that are positive for cFn binding are also positive for binding to rEDA. In contrast, binding of the rlll4 to rScl1 proteins was comparable to that of the negative control, P163, for all three ECM ligands. When increasing concentrations $(0.02-4.0 \mu \mathrm{M})$ of ECM ligands cFn, rEDA and rlll4 were incubated with immobilized $\mathrm{rScl}$ proteins to assess binding specificity, concentration-dependent binding of cFn and rEDA to all three rScl1 constructs was observed, plateauing around the 3-4 $\mu \mathrm{M}$ concentration (Fig. 1C). In contrast, no binding by rlll4 to rScl1 constructs was observed over the entire concentration range nor was binding by any ECM ligand to the negative control, P163, detected (data not shown). We also observed that rScl1 proteins bind better to cFn than rEDA construct. One possible explanation is that the EDA conformation in cFn and rEDA preparations is not identical or that rScl1 binding alters cFn structure (53), resulting in improved binding. The latter mechanism would not be surprising since it has been reported for Fn binding by other bacterial adhesins, including streptococcal (M, F1/Sfbl) and staphylococcal (FnbpA) proteins, as well as the BBK32 protein of Borrelia burgdorferi $(21,34,40,55)$. Altogether, our data indicate that the Scl1-cFn interaction is mediated, at least in part, via the EDA domain, and that this interaction is specific and concentration-dependent. 
Mapping of Scl1 binding site within EDA

The cFn/EDA spliced variant produced in response to tissue injury $(8,25,74,77)$ includes a loop region between the $C$ and $C^{\prime} \beta$-strands (Fig. 2A), which mediates binding to $\alpha_{4} \beta_{1}$ and $\alpha_{9} \beta_{1}$ integrins that actively participate in wound healing (75). Since loop regions are often involved in protein-protein interactions, we hypothesized that the C-C' loop region of EDA contains the Scl1 binding site. To test this, we examined the ability of an exogenous synthetic eleven amino-acid peptide (TYSSPEDGIHE), corresponding to the C-C' loop region of EDA, to inhibit binding of ECM ligands to immobilized rScl1 proteins, P176, P144, and P161. In this assay, increasing concentrations $(0.01 \mathrm{mM}, 0.1 \mathrm{mM}$, and $1.0 \mathrm{mM})$ of the C-C' loop peptide or a scrambled peptide control (SEDIHYTEGPS) were pre-incubated with immobilized $\mathrm{rScl}$ proteins prior to the addition of the ECM ligands, cFn and rEDA. As shown in Fig. 2B, binding of cFn or rEDA to all three rScl1 proteins was inhibited by the C-C' loop peptide in a concentrationdependent manner up to $50 \%$ and $60 \%$ respectively, whereas no inhibition was observed with the scrambled peptide. These results mapped a Scl1 binding site to the EDA C-C' loop region.

Previous studies reported that residues $\mathrm{Asp}^{41}$ and Gly ${ }^{42}$ (Fig. 2A) within the CC' loop region of EDA are involved in $\alpha_{9} \beta_{1}$-integrin binding and that the monoclonal antibody IST-9 binds the C-C' loop of EDA at adjacent residues $\| \mathrm{e}^{43}$ and $\mathrm{His}^{44}$, and blocks this integrin engagement $(47,75)$. We therefore used the anti-EDA mAb IST-9 to determine if Scl1 and $\alpha_{9} \beta_{1}$ integrin have overlapping binding sites within the EDA C-C' loop region (Fig. 2C). Both cFn and rEDA were 
pre-treated with increasing concentrations $(0.1 \mu \mathrm{g}, 1.0 \mu \mathrm{g}$, and $10 \mu \mathrm{g})$ of IST-9 before mixtures were added to and incubated in the wells with immobilized rScl1 proteins. With increasing concentrations of IST-9 mAb, we show a significant inhibition of binding by both cFn (10-45\%) and rEDA (20-54\%) to all three rScl1 constructs. These data mapped a Scl1 binding site within the C-C' loop region of EDA, which is also recognized by the $\alpha_{9} \beta_{1}$ integrin.

The rScl1-EDA interaction represents a common cFn-binding mechanism among pathogenically varying GAS strains

Previous work showed that Scl1 and Scl2 proteins are related but differ in their $C L$ region length and type of triplet repeats used $(30,87)$. Likewise, phylogenetic tree of the Scl1- and Scl2-V regions revealed that they form two separate branches but are evolutionary related (29). The V-region sequences also differ among Scl1 and Scl2 variants found in various GAS strains and they are typically M-type specific $(49,50,66,67,86)$. To date, majority of human ligands bind to Scl1-V but not Scl2-V regions and they display two main binding patterns e.g., some bind factor $\mathrm{H}(\mathrm{CFH})$ and factor $\mathrm{H}$-related protein 1 (CFHR1)(12, 68), whereas other bind low-density lipoprotein (29) and ECM components, cFn and Lm (13). To test whether cFn binding via EDA was conserved among Scl1 variants originated from GAS strains of various $M$ types, we examined all previously tested rScl constructs (12) for binding to rEDA. In this assay, we used the rScl1 proteins, including P144, P161, P176, P186, P190, and P217 that were positive for binding to $\mathrm{cFn}$ and Lm, as well as the CFH/CFHR1-binding positive 
rScl1 proteins $\mathrm{P} 179$ and P216. In addition, the rScl2 proteins P163, P177, and P178 were used as ligand-binding negative controls. As shown in Fig. 3A, rScl1 binding to cFn corresponded with binding to rEDA, whereas no binding to $\mathrm{pFn}$ by any rScl1 or rScl2 proteins was observed. To test whether all rScl1 constructs bind EDA via the same mechanism, we examined binding of cFn and rEDA in the C-C' peptide inhibition assay described above. The C-C' loop peptide was able to compete with both cFn and rEDA binding to rScl1 proteins, decreasing binding by $50-70 \%$, with no inhibition detected by the scrambled peptide (Fig. 3B). As expected, no additional binding inhibition was observed by the C-C" loop peptide for $\mathrm{cFn}$ - and rEDA-binding negative rScl1 and $\mathrm{rScl} 2$ proteins. Peptide inhibition results were further strengthened by the IST-9 antibody blocking assay, which was also found to inhibit binding of cFn and rEDA 50\% and beyond. The data provide evidence that $\mathrm{cFn}$ binding via the C-C' loop region of EDA is conserved among pathogenically varying GAS strains expressing the ECM-binding Scl1 variants.

\section{The Scl1-ECM interaction supports GAS biofilm formation}

Scl1 proteins have been found to mediate GAS biofilm formation on an abiotic surface (60). To assess the importance of the Scl1-cFn interaction in GAS biofilm formation, cell biomass produced by three epidemiologically diverse M41-, M28-, and M1-type GAS strains was compared spectrophotometrically following crystal violet staining in an early adhesion $(1 \mathrm{~h})$ and mature biofilm $(24 \mathrm{~h})$ in untreated or cFn-treated wells (Fig. 4A). M41 wild-type (WT) and the available M41scl1- 
complemented (sc/1-C) mutant strains form a more robust biofilm at 24 h (O.D.600 $\sim 0.5$ untreated vs. $\sim 0.7 \mathrm{cFn}$-coated), as compared to M28 WT ( 0.45 untreated v. $\sim 0.6 \mathrm{cFn}$-coated) and M1 WT ( 0.23 untreated; $\sim 0.34 \mathrm{cFn}$-coated) strains. Importantly, in each case, more bacterial biomass was detected in cFn-treated wells than on abiotic surface during both the early adherence stage $(1 \mathrm{~h})$, as well as in mature biofilms (24 h). Finally, we show a statistically significant decrease in biofilm forming capacity by all Scl1-negative isogenic mutants as compared to their parent strains at both time points. Confocal laser scanning microscopy (CLSM) of $24 \mathrm{~h}$ biofilms confirmed crystal violet staining results (Fig. 4B). The M41 WT biofilm was the most robust with a thickness averaging $26 \mu \mathrm{m}$, while a nearly $50 \%$ decrease was observed for the M41scl1 mutant $(15 \mu \mathrm{m})$. A similar pattern is shown for the M28 WT (22 $\mu \mathrm{m})$ over the scl1 mutant $(14 \mu \mathrm{m})$. The M1type WT GAS $(15 \mu \mathrm{m})$ has a decreased capacity for biofilm formation with M1sc/1 mutant $(7 \mu \mathrm{m})$ poorly supporting a biofilm phenotype. When compared to GAS biofilms formed in untreated (abiotic) wells by the same strains (60), cFnmediated biofilms were enhanced up to $40 \%$ in average thickness recorded for wild-type strains. Collectively, we can conclude from these studies that Scl1mediated binding of GAS to cFn significantly enhances biofilm formation.

Scl1-mediated binding to fibroblast-derived ECM supports GAS adherence and biofilm formation

To develop a system that allows biofilm development on ECM more closely mimicking the ECM in tissue in vivo, normal human dermal fibroblasts (HDFa) 
were used to deposit extracellular matrix. To generate the fibroblast-derived ECM (fdECM), wells were denuded of HDFa cells and the presence of fdECM was visualized by Ponceau S staining (Fig. S1A). When the composition of fdECM coating was characterized by ELISA using antibodies that detect ECM proteins, fibronectin and collagens were identified as major constituents (Fig. S1B). Importantly, we detected the presence of the EDA/cFn variant within fdECM using the monoclonal antibody, IST-9. Thus, we confirmed that fdECM coating provides a more complex model of tissue matrix compared to single ECM component, which includes the EDA/cFn population recognized by Scl1, a streptococcal adhesin.

GAS strains used in this study express varying numbers of surface adhesins, including fibronectin-binding adhesins. The fdECM matrix contains a number of ECM components assembled into a higher-ordered structure more suitable for assessing whether Scl1-EDA/cFn binding significantly contributes to the adherence and biofilm formation by epidemiologically varying GAS strains. The structural complexity of the fdECM network was demonstrated by field emission election microscopy (FESEM) (Fig. 5A, row (i)). Using FESEM to image GAS adherence on a fdECM coating, wild-type GAS strains of M1- (ii), M28- (iii), and M41-type (iv) were observed to be targeted or preferentially bound to fdECM deposits, as compared to the surrounding abiotic surface at early time points in infection. At higher magnification, GAS chains are observed in close contact with fdECM fibrous structures. When GAS WT and scl1-inactivated mutant strains were compared based on crystal violet staining for bacterial biomass after $1 \mathrm{~h}$ 
(adherence) and $24 \mathrm{~h}$ (mature biofilm) on fdECM, or cFn-coated wells, GASfdECM binding produced comparable biofilm absorbance values to those of the cFn-treated wells (Fig. 5B). Scl1-negative isogenic mutants had decreased capacity for adherence and mature biofilm formation, as compared to their WT parent strains, on both ECM-treated wells. Our findings demonstrated that the Scl1 protein plays a significant role in GAS biofilm formation on a single cFn coating, as well as on a more complex fdECM, for all strains tested.

Experiments shown in Figs. 2-3 demonstrated that we can significantly inhibit rScl1 binding to both rEDA and cFn using the synthetic C-C' loop peptide or IST9 mAb. Here, we adapted both inhibition assays in order to evaluate the importance of the interactions between native Scl1 surface protein and EDA/cFn component of fdECM in total GAS adherence (Fig. 6). Graphs represent spectrophotometric measurements following crystal violet staining $\left(\mathrm{OD}_{600}\right.$ values) obtained for each WT strain (M41, M28, and M1) on all three ECM coatings (cFn, rEDA, fdECM) in wells without inhibitors and in wells treated with either the C-C' loop peptide (panel A) or IST-9 mAb (panel B). Below the graphs, we present these $\mathrm{OD}_{600}$-values data as percent (\%) adherence inhibition, as compared with their corresponding untreated samples (100\% adherence). For comparison, we also present \% adherence inhibition, resulting from genetic inactivation, of their corresponding untreated scl1 mutants $\left(\mathrm{OD}_{600}\right.$ values are shown in Fig. S2). First, the C-C' loop or scrambled peptide (1 mM each) were added to GAS WT (Fig. 6 A) or scl1 mutant strains (Fig. S2A). Next, peptide-GAS mixtures were added to ECM-coated wells and $1 \mathrm{~h}$ adherence was analyzed spectrophotometrically 
following crystal violet staining. The adherence of all WT strains was significantly inhibited on all three coatings, whereas adherence of sc/1-inactivated mutants was unaffected by peptide treatment (Fig. S2). Inhibition levels of adherence obtained for peptide-treated strains type M41 and M28 were similar, ranging on assorted ECM coatings between $25-27 \%$ of their untreated samples. The remaining binding levels (as well as \% inhibition values) were similar to the binding levels of untreated Scl1-negative mutant strains, ranging between 24 $28 \%$, which is depicted in the graph by horizontal lines over-imposed on bars representing untreated samples. Interestingly, a 25-33\% C-C' peptide inhibition range was also measured for the M1 WT strain; however, the binding-inhibition levels obtained for the M1-sc/1 mutant on assorted ECM coatings were substantially higher (51-54\%). No strains were inhibited by treatment with the scrambled peptide (data not shown).

In a second set of inhibition studies (Fig. 6B, Fig. S2B), ECM-coated wells were pre-treated with $10 \mu \mathrm{g}$ of mAb IST-9 for $1 \mathrm{~h}$ followed by the addition of GAS strains. Again, $1 \mathrm{~h}$ adherence was analyzed spectrophotometrically following crystal violet staining. Similar to the peptide inhibition described above, we identified a statistically significant decrease in adherence by M41 and M28 WT strains treated with IST-9 mAb (24-30\% range) and, again, the remaining binding levels of these IST-9-treated samples were similar to the binding levels of their untreated Scl1-negative mutants (25-32\%). As previously shown, by comparison, the M1 scl1 mutant had substantially decreased adherence on all three ECM coatings (50-60\% inhibition), whereas adherence inhibition of the M1 WT strain 
due to IST-9 treatment was lower (32-35\%). In total, inhibition studies demonstrate that GAS adherence to ECM substrates, including complex fdECM network, can be abrogated with treatments that target the integrin-binding C-C' loop region of the EDA/cFn.

\section{Discussion}

The skin is an organ covering the human body that forms an effective barrier between the internal and external environments and protects against invading microbes (33). Group A Streptococcus (GAS) is, together with Staphylococcus aureus, a predominant pathogen of the skin and soft tissue $(7,85)$. GAS, as well as other bacteria, requires a portal of entry to initiate the infection. In the wounded site, GAS faces a new environment, which creates a unique opportunity for adhesion to the host's cellular and extracellular components via surface adhesins designated MSCRAMMs for 'microbial surface components recognizing adhesive matrix molecules (65). Among various MSCRAMMs expressed by GAS strains (14), Scl1 was recently shown to selectively bind cFn (13). Here, we characterize Scl1-cFn interactions and investigate the role of this binding in GASECM adherence and biofilm formation on single ECM coatings and on complex ECM structures deposited by human cells.

In the present study, we selected three model GAS strains representing the intraspecies breadth. The M1-type strain represents the global M1T1 clone responsible for pharyngitis and invasive infections $(4,78)$, the M28-type strain has been historically associated with puerperal sepsis $(27,28,58)$, and the M41- 
type strain has been predominantly linked to skin infections $(3,22,23)$. Importantly, these strains vary in the number of fibronectin (Fn)-binding proteins they express in addition to Scl1 (10). The M28-type strain harbors at least three genes encoding known Fn-binding proteins, including the serum opacity factor (sof) $(17,39,59)$, prtF1/sfbl (31) and prtF2/pfbp $(37,70)$. M41-type strain has the prtF2/pfbp gene, whereas the M1-type strain has none of them. Based on this knowledge, the contribution of each Scl1.1, Scl1.28, and Scl1.41 variant to the attachment and biofilm formation on single cFn and complex fdECM coatings by these strains was tested.

First, we investigated the selective recognition of and binding to cFn by rScl1 proteins. There is a large body of literature describing redundancy in Fn binding by GAS surface proteins (32). They are often closely related among Grampositive bacteria or contain related regions that are involved in Fn binding (65) and include GAS proteins M, F1/Sfbl, Sfbll, F2/PFBP, SOF, FBP54, and FbaA and $\mathrm{B}(6,17,21,37,43,44,79,82,83)$. Unlike Scl1, these proteins bind $\mathrm{pFn}$ and subsequent studies mapped their binding sites within the $\mathrm{N}$-terminal region spanning type I and type II repeats of Fn, which is identical in pFn and cFn (18, $24,54,61)$. The cFn isoforms differ from $\mathrm{pFn}$ in that they include in varying proportions the extra domains, termed EDA/EIIIA and EDB/EIIIB (64). In the wound, platelets are activated, degranulate and release EDA-containing Fns into the apical aspect of the fibrin matrix in the region that is first encountered by microorganisms (71). Within two days wound macrophages and fibroblasts produce cFns rich in the EDA segment and these cFns persist throughout the 
wound bed for at least a week following injury (8). Using peptide inhibition and antibody blocking experiments, we demonstrated that multiple rScl1 proteins bind cFn via the C-C' loop region of EDA, indicating a common binding mechanism among pathogenically discrete strains. Thus, we have identified a novel Fnbinding mechanism unique to Scl1, which allows GAS to target the EDA/cFn spliced variant expressed in wounded tissue.

Within hours of injury, neutrophils populate the wound and thereafter macrophages arrive and epidermal keratinocytes commence migration over the provisional matrix (56). These cells express integrins that bind the EDA/cFn, including $\alpha_{9} \beta_{1}(80)$. The C-C' loop region of EDA domain mediates binding to $\alpha_{9} \beta_{1}$ and $\alpha_{4} \beta_{1}$ integrins (Shinde and Van De Water, unpublished observations) and has been shown to participate in cell adhesion, cell spreading (75) and matrix assembly (5). Aberrations in, or blocking of, the C-C' loop region in EDA abrogates the EDA-integrin binding $(46,75)$. Our current binding-inhibition studies, as well as preliminary cell-attachment inhibition assays (not shown), demonstrated that $r S c l 1$ binds to the $C_{-} C^{\prime}$ functional loop region of EDA, which is also the integrin-binding site. This implies that during GAS infection, the Scl1 adhesin could abrogate human cell migration and adhesion, thus, impairing wound healing. Interestingly, a recent study showed that reepithelialization of a murine cutaneous wound was delayed by staphylococcal infections (72). Considering the overall similarities between streptococcal and staphylococcal skin infections, it is tempting to speculate that GAS evolved analogous molecular 
mechanisms that augment tissue colonization by manipulating the local host's environment at the site of infection.

GAS biofilm is a relatively new concept (45), which is increasingly being established with supporting data collected from clinical specimens (1) and through animal model research $(16,69)$. Several GAS surface components have been reported to participate in biofilm formation $(15,19,41,51,52)$ including the Scl1 protein (60). In the latter study, we reported that Scl1 plays a substantial role in GAS biofilm formation on an abiotic surface. We also proposed a model in which Scl1-ECM binding enhances biofilm formation by anchoring the growing biofilm structure. Here, we tested that model by assessing the significance of one interacting component, Scl1-cFn binding in the formation of GAS biofilm. Direct comparison of biofilms grown by the M1-, M28-, and M41-type strains showed a significantly increased biofilm biomass on cFn-coated surface compared to an inanimate surface. These differences were further confirmed microscopically since the average thickness of mature biofilms formed by each of these strains was higher on cFn coating by up to $40 \%$. However, these results could also be explained by the non-Scl1-mediated interactions between $\mathrm{cFn}$ and other $\mathrm{Fn}$ binding MSCRAMMs expressed by these strains. Therefore, a similar comparison was made between biofilms formed by the wild-type and isogenic scl1-inactivated mutants of each M-type strain on cFn coating. Despite the presence of additional Fn-binding proteins, especially in M28- and M41-type strains, that bind a different region of Fn, all Scl1-devoided mutants produced 
significantly lower biofilm biomass and lower biofilm thickness by nearly $50 \%$, suggesting an important role for the Scl1-EDA interaction.

The complexity of natural ECM in tissue exceeds the single cFn coating we initially employed. Therefore, we developed a more complex fibroblast-derived ECM coating (fdECM), which was denuded of cells. Following eukaryotic cell removal, tissue culture wells contained a complex 2-D fibrillar network composed of several ECM components, including various collagens and laminin in addition to the EDA/cFn isoforms within fibronectin. A similar approach was used to study the attachment and biofilm formation of Klebsiella pneumoniae on coating derived from ECM deposited by bronchial epithelial cells (38). This coating produced a rich biofilm phenotype that was more versatile as compared to a collagen coating or an abiotic surface. In our hands, complex fdECM supported wild-type GAS adherence and biofilm formation at the levels comparable with cFn coating. Scanning electron microscopy revealed targeted or preferential GAS binding to fdECM structures over the surrounding abiotic area. The $s c / 1$ isogenic mutants showed significantly decreased adherence and biofilm formation on fdECM similarly to that on cFn-treated wells. Furthermore, we were able to reduce the adherence of the wild-type strains to the levels shown by the $s c / 1$ mutants with the C-C' loop peptide and mAb IST-9. The importance of Scl1EDA/cFn binding in GAS adherence on fdECM was intriguing considering the fact that GAS cells are equipped with more than one Fn-binding protein and, likely, with more than one type of MSCRAMM targeting additional components of fdECM. Since inhibition was not complete, our data also point at the contribution 
and importance of other adhesins and alternative ECM-binding mechanisms to GAS adherence and host colonization.

In summary, we characterized Scl1-cFn interactions and mapped the Scl1 binding site within the functional C-C' loop region of the EDA segment overlapping with the $\alpha_{9} \beta_{1}$-integrin binding site. This observation opens new directions of investigations since $\alpha_{9} \beta_{1}$ is expressed by a number of epithelial and inflammatory cells involved in tissue repair and wound healing $(63,76,77,80)$. We studied the contribution of Scl1-ECM adhesion in GAS biofilm formation on a single cFn coating and on complex fdECM 2-D matrix. Based on these results, we propose a model of the Scl1-mediated tissue colonization by GAS (Fig. 7). In this mechanistic model, the opportunistic GAS pathogen exploits injured tissue as a portal of entry to establish skin infection. Within injured site, platelets, macrophages, and underlying injured fibroblasts deposit in the wound bed a provisional ECM matrix (proECM), which includes the EDA-enriched cFn to provide scaffolding for cell migration and proliferation via $\alpha_{9} \beta_{1}, \alpha_{4} \beta_{1}$ and $\alpha_{4} \beta_{7}$ integrins, and promote structural remodeling and tissue repair (35). Upon entry into the injured site, GAS utilizes the Scl1 protein to initiate binding to the EDA domain of $\mathrm{cFn}$, which is mediated via the C-C' loop region and secures adherence to ECM, facilitating microcolony formation. Scl1 acts in concert with other GAS adhesins that, depending on strain, may include Fn-binding proteins M, F1/Sfbl, Sfbll, F2/PFBP, SOF, FBP54, and FbaA and B $(6,17,21,37,43,44$, 47, 73, 79, 82). The Scl1-EDA binding may also compete with EDA binding by the integrins expressed on migrating keratinocytes, thus, delaying wound 
reepithelialization and healing. Growing GAS microcolonies form a focused nidus of tissue infection until it is overcome by immune defenses or disperses to new superficial or deep tissue sites. Validation of this updated model is ongoing research.

\section{Experimental procedures}

\section{Bacterial strains and growth}

The M41- (MGAS 6183), M28- (MGAS 6143), and M1-type (MGAS 5005) strains of group A Streptococcus were used. All GAS cultures were grown at $37^{\circ} \mathrm{C}$ in an atmosphere of $5 \% \mathrm{CO}_{2}-20 \% \mathrm{O}_{2}$. For biofilm formation, GAS strains were grown overnight on brain-heart infusion agar (BHI) (BD Biosciences) and used to inoculate Todd-Hewitt broth (Difco) supplemented with $0.2 \%$ yeast extract (THY medium). Cultures were incubated until they reached logarithmic phase (O.D. 600 $\sim 0.5$ ) and used to inoculate treated or untreated tissue culture wells.

\section{Construction of scl1-inactivated mutants}

The isogenic Scl1-negative mutants for all wild-type strains were constructed by allelic replacement as described previously. Briefly, the scl1 gene in M1-type strain was inactivated using the suicide plasmid, pSL134, harboring the sc/1.1 allele with flanking regions and nonpolar spectinomycin resistance cassette, spc2, inserted in-frame to replace the $s c / 1.1$ coding region (48). The scl1.28 and scl1.41 alleles were inactivated using the pSL170 suicide plasmid containing the erythromycin resistant sc/1.28::erm2 construct $(10,29)$. Wild-type strains were 
electroporated followed by incubation in THY medium for 2.5 hours. Cultures were then plated onto $\mathrm{BHI}$ agar containing either $100 \mathrm{~g} \mathrm{ml}^{-1}$ of spectinomycin or $3 \mu \mathrm{g} \mathrm{ml}^{-1}$ of erythromycin. Drug-resistant transformants were initially screened by PCR for amplified single products, indicating double cross-over recombination, and were subsequently confirmed by DNA sequencing. For complementation of the scl1.41-inactivated mutant, the MGAS 6183 scl1-mutant cells were electroporated with the plasmid construct pSL230 encoding the Scl1.41 protein (10). Scl1 expression or lack of it was confirmed by western immunoblotting.

\section{Recombinant proteins}

Several sc/1 and scl2 alleles encoding the extracellular protein portion were amplified by PCR and cloned into the PASK-IBA2 vector designed for periplasmic protein expression $(10,29,30,36,87)$. Recombinant Scl1 and Scl2 (rScl) proteins used in these assays: P144/rScl1.1 (accession no. AF252861), P161/Scl1.28 (accession no. AY459361), P176/rScl1.41 (accession no. AY452037), P179/rScl1.6 (accession no. EU 127997) P186/rScl1.12 accession no. DQ309441), P190/rScl1.2 (accession no. EU127996), P216/rScl1.55 (accession no. EU127999), P217/rScl1.52 (accession no. EU127998), P163/rScl2.28 (accession no. AY069936), P177/rScl2.4 (accession no. DQ309442), P178/rScl2.77 (accession no. DQ309443). All recombinant Scl proteins contain short C-terminal tag, the Strep-tag II (WSHPQFEK), which has high binding affinity to Strep-Tactin-Sepharose for affinity chromatography 
purification (IBA-GmbH, Goettingen, Germany). E. coli DH5 $\alpha$ and BL21 strains were used for cloning and protein expression, respectively.

The his-tagged recombinant EDA (rEDA) and III4 (rllI4) polypeptides were prepared as described (47). Briefly, EDA or III4 cDNA was amplified by PCR, cloned into plasmid pGEX-2T, expressed in E. coli, and purified using affinity chromatography nickel columns.

\section{Protein binding assays}

For binding studies, rScl proteins $(0.5 \mu \mathrm{M})$ were immobilized onto Strep-Tactincoated microplate wells for $1.5 \mathrm{~h}$ at room temperature and blocked with Trisbuffered saline (TBS) supplemented with $1 \%$ bovine serum albumin (BSA) overnight at $4^{\circ} \mathrm{C}$ followed by incubation with the extracellular matrix (ECM) proteins cellular fibronectin (cFn), rEDA, and rlll4 or plasma fibronectin (pFn)(Sigma). The no rScl controls were performed in BSA-coated wells for each ligand and each antibody used. Final ODs were normalized by subtracting the BSA controls in each experimental setup.

All ECM ligands prepared at $0.25 \mu \mathrm{M}$ concentration for binding inhibition studies or $1 \mu \mathrm{g}$ of ECM ligand per well for protein screening experiments, were added to rScl-immobilized wells in triplicate and incubated at room temperature for $1 \mathrm{~h}$. Bound ligands were detected with specific anti-cFn (Sigma) and anticollagen I, -collagen II, -collagen IV primary antibodies and goat-anti-rabbit secondary antibodies conjugated to horseradish peroxidase (HRP)(BioRad). The 
HRP substrate, 2,20-azino-bis(3-ethylbenzthiazoline-6-sulfonic acid) was used and colorimetric reaction was recorded at O.D. $415 \mathrm{~nm}$.

For peptide inhibition studies, synthetic 11 -amino-acid peptide representing the C-C' loop region of the EDA domain (TYSSPEDGIHE) or a scrambled loop peptide (SEDIHYTEGPS) were used (LifeTein, Inc.). rScl proteins $(0.5 \mu \mathrm{M})$ were immobilized onto Strep-Tactin-coated wells as above and were either untreated or were pre-incubated with increasing concentrations $(0.01-1.0 \mathrm{mM})$ of the synthetic C-C' loop EDA peptide or scrambled peptide for 30 minutes to $1 \mathrm{~h}$ at room temperature. cFn or rEDA ligands were next added to the wells and incubated at room temperature for $1 \mathrm{~h}$. Bound ECM ligands were detected with specific primary and HRP-conjugated secondary antibodies as above.

For antibody inhibition studies, the monoclonal antibody IST-9 was used,

which recognizes the $\mathrm{Il}^{43}$ and His ${ }^{44}$ epitope (47) within the C-C' loop region of EDA. ECM ligands $(0.25 \mu \mathrm{M})$ were pre-incubated with increasing concentrations $(0-10 \mu \mathrm{g})$ of the IST $-9 \mathrm{mAb}$ for 30 minutes at room temperature. Mixtures were added to rScl1-immobilized wells and bound ECM ligands were detected as above with specific primary and HRP-conjugated secondary antibodies.

\section{Analysis of biofilm formation}

Crystal violet staining assay. Untreated or pre-treated tissue culture polystyrene 24-well plates were used to assess biofilm formation. ECM-coated wells were pre-treated with cellular fibronectin, rEDA or $1 \% \mathrm{BSA}$ in TBS overnight at $4^{\circ} \mathrm{C}$ and then blocked for $2 \mathrm{~h}$ with 1\% BSA in TBS at room temperature. Exponential- 
phase GAS cultures $(0.5 \mathrm{ml})$ were seeded into untreated or treated wells and incubated at $37^{\circ} \mathrm{C}$ in an atmosphere of $5 \% \mathrm{CO}_{2}-20 \% \mathrm{O}_{2}$. Medium was removed at $1 \mathrm{~h}$ or $24 \mathrm{~h}$ and wells were washed with PBS followed by the addition of $0.5 \mathrm{ml}$ of $1 \%(\mathrm{v} / \mathrm{v})$ solution of crystal violet reagent (Becton Dickinson) in PBS, and incubation at room temperature for 30 minutes. Biomass staining was solubilized with $0.5 \mathrm{ml}$ of ethanol and spectrophotometric readings were recorded at O.D. 600 $\mathrm{nm}$ for each sample at each time point. Statistical analysis is reported based on three independent experimental repeats $(n=3 \pm S D)$ with triplicate technical replicates in each experiment. Statistical significance was denoted as ${ }^{*} P \leq 0.05$ and ${ }^{* *} \mathrm{P} \leq 0.001$.

Adherence inhibition studies. For inhibition of biofilm formation both synthetic CC' loop peptide and IST-9 mAb were used. GAS WT and mutant strains were grown to logarithmic phase and pre-incubated with $1 \mathrm{mM}$ synthetic C-C' loop or scrambled peptide. $0.5 \mathrm{ml}$ of the mixture was then added to ECM-treated wells in triplicate and incubated at $37^{\circ} \mathrm{C}$ in an atmosphere of $5 \% \quad \mathrm{CO}_{2}-20 \% \quad \mathrm{O}_{2}$. Adherence inhibition at $1 \mathrm{~h}$ was analyzed spectrophotometrically following crystal violet staining. For antibody inhibition assay, ECM-treated wells were preincubated with mAb IST-9 $(10 \mu \mathrm{g})$ for $1 \mathrm{~h}$ at room temperature. Wells were then washed with PBS to remove unbound antibody. $0.5 \mathrm{ml}$ of logarithmic phase GAS wild-type and mutant strains were added to triplicate wells and incubated at 37 ${ }^{\circ} \mathrm{C}$ in an atmosphere of $5 \% \mathrm{CO}_{2}-20 \% \mathrm{O}_{2}$. Bacterial biomass was measured spectrophotometrically after $1 \mathrm{~h}$ following crystal violet staining. . Statistical 
analysis is reported based on three independent experimental repeats $(n=3 \pm$ SD) with triplicate technical replicates in each experiment. Statistical significance was denoted as ${ }^{*} \mathrm{P} \leq 0.05$ and ${ }^{* *} \mathrm{P} \leq 0.001$.

\section{Microscopy Techniques}

Confocal Laser Scanning Microscopy (CLSM). To visualize GAS cells by CLSM, GAS wild-type and sc/1-inactivated mutants were transformed with plasmid pSB027 $(13,20,60)$ to express green fluorescent protein (GFP). GAS cells grown to O.D. $600 \mathrm{~nm} \sim 0.5$ were inoculated onto untreated or cFn-coated $15-\mathrm{mm}$ glass cover slips placed into 24-well tissue culture plate wells and incubated for $24 \mathrm{~h}$. Biofilms were washed gently with PBS and fixed with 3\% paraformaldehyde in PBS at room temperature for at least $2 \mathrm{~h}$. Cover slips were mounted to slides using Prolong Gold (Invitrogen). Fluorescent images were acquired using the confocal microscope (LSM 510 Carl Zeiss) equipped with a 63x/1.40 Oil PlanApochromatic objective. Z-stack profiles were further deconvoluted stepwise by AutoQuant software at $0.36 \mu \mathrm{m}$ and were visualized as 3-dimensional images using NIS - Elements software. Additional visualization was performed using the Zeiss trinocular EPI fluorescence microscope equipped with four achromatic objectives. The Zeiss AxioCam Mrc5 camera and Zeiss AxioVision 4.8 software was used for image acquisition.

Field Emission Scanning Electron Microscopy (FESEM). GAS biofilm samples were prepared and fixed as above. The samples were post-fixed in osmium tetroxide, dehydrated in an ethanol series, and dried using hexamethyldisalizane. 
Samples were mounted onto aluminum stubs, sputter-coated with gold/palladium, and imaged on a Hitachi S-4800 field emission scanning electron microscope as previously reported (60).

Cell culture techniques

Fibroblast-derived extracellular matrix (fdECM) production and analysis. Adult human dermal fibroblasts (HDFa) were grown in Medium 106 (Invitrogen) supplemented with low serum growth supplement to confluency at $37^{\circ} \mathrm{C}$ in an atmosphere of $5 \% \mathrm{CO}_{2}$. For fdECM production, HDFa were grown on sterile 15 $\mathrm{mm}$ glass cover slips inserted into wells. Cells were removed by treatment with 2 $\mathrm{mM}$ ethylene glycol tetraacetic acid, leaving naturally deposited network of extracellular matrix. Samples were washed gently with PBS and fixed with 3\% paraformaldehyde for at least $2 \mathrm{~h}$ or overnight, and analyzed microscopically following Ponceau S staining or by FESEM. ELISA was performed to determine the presence of extracellular matrix components: total cellular fibronectin, EDAcontaining cFn, collagens type I, II, and IV, and laminin. Components present were detected with specific primary antibodies: anti-Fn (Sigma),anti-EDA/cFN (IST-9: Santa Cruz Biotechnology) anti-collagens I, II, IV (Chemicon/Millipore), and anti-laminin (Sigma). Appropriate secondary antibodies conjugated to horseradish peroxidase (BioRad) were used and developed with ABTS substrate. 


\section{Statistical analysis}

Statistical analysis was performed using the two-tailed paired Student's $t$-test and significance was denoted at a level of ${ }^{*} P \leq 0.05$ or ${ }^{* *} P \leq 0.001$. Error bars represent standard deviations with analyses based on three independent experimental repeats $(n=3)$, each performed in triplicate technical replicates.

\section{Acknowledgements}

We thank Joan Olson and Fred Minnear for the critical reading of the manuscript. The support of Xin-Xing Gu is greatly appreciated. This work was supported in part by National Institutes of Health Grant Al50666 (to S. Lukomski) and GM056442 (to L. Van De Water). Authors also wish to acknowledge the WVU Research Office's Program to Stimulate Competitive Research (PSCoR), as well as the Research Funding Development Grant (RFDG) (to S. Lukomski). H. Oliver-Kozup was supported by a grant from the West Virginia Graduate Student Fellowship in Science, Technology, Engineering and Mathematics (STEM). Confocal microscopy experiments were performed in the West Virginia University Microscope Imaging Facility, which has been supported by the Mary Babb Randolph Cancer Center and NIH grants P20 RR016440, P30 RR032138/GM103488 and P20 RR016477. The findings and conclusions in this report are those of the authors and do not necessarily represent the views of the National Institute of Occupational Safety and Health. 


\section{References}

1. Akiyama, H., S. Morizane, O. Yamasaki, T. Oono, and K. Iwatsuki. 2003. Assessment of Streptococcus pyogenes microcolony formation in infected skin by confocal laser scanning microscopy. J Dermatol Sci 32:193-199.

2. Almengor, A. C., M. S. Walters, and K. S. McIver. 2006. Mga is sufficient to activate transcription in vitro of $s o f-s f b X$ and other Mga-regulated virulence genes in the group A Streptococcus. Journal of Bacteriology 188:2038-2047.

3. Anthony, B. F., L. V. Perlman, and L. W. Wannamaker. 1967. Skin Infections And Acute Nephritis In American Indian Children. Pediatrics 39:263-279.

4. Aziz, R., and M. Kotb. 2008. Rise and persistence of global M1T1 clone of Streptococcus pyogenes. Emerg Infect Dis 14:1511-1517.

5. Bazigou, E., S. Xie, C. Chen, A. Weston, N. Miura, L. Sorokin, R. Adams, A. F. Muro, D. Sheppard, and T. Makinen. 2009. Integrin-alpha9 is required for fibronectin matrix assembly during lymphatic valve morphogenesis. Dev Cell 17:175-186.

6. Bingham, R. J., E. Rudino-Pinera, N. A. Meenan, U. Schwarz-Linek, J. P. Turkenburg, M. Höök, E. F. Garman, and J. R. Potts. 2008. Crystal structures of fibronectin-binding sites from Staphylococcus aureus FnBPA in complex with fibronectin domains. Proc Natl Acad Sci U S A 105:12254-12258.

7. Bisno, A. L., F. A. Rubin, P. P. Cleary, and J. B. Dale. 2005. Prospects for a Group A Streptococcal Vaccine: Rationale, Feasibility, and Obstacles--Report of a National Institute of Allergy and Infectious Diseases Workshop. Clin Infect Dis 41:1150-1156.

8. Brown, L. F., D. Dubin, L. Lavigne, B. Logan, H. F. Dvorak, and L. Van de Water. 1993. Macrophages and fibroblasts express embryonic fibronectins during cutaneous wound healing. Am J Pathol 142:793-801.

9. Carapetis, J., A. Steer, E. Mulholland, and M. Weber. 2005. The global burden of group A streptococcal diseases. Lancet Infect Dis 5:685-694.

10. Caswell, C., E. Lukomska, N. Seo, M. Höök, and S. Lukomski. 2007. Scl1-dependent internalization of group A Streptococcus via direct interactions with the $\alpha_{2} \beta_{1}$ integrin enhances pathogen survival and re-emergence. Mol Microbiol 64:1319-1331.

11. Caswell, C. C., M. Barczyk, D. R. Keene, E. Lukomska, D. E. Gullberg, and S. Lukomski. 2008. Identification of the first prokaryotic collagen sequence motif that mediates binding to human collagen receptors, integrins $\alpha_{2} \beta_{1}$ and $\alpha_{11} \beta_{1}$. J Biol Chem 283:36168-36175.

12. Caswell, C. C., R. Han, K. M. Hovis, P. Ciborowski, D. R. Keene, R. T. Marconi, and S. Lukomski. 2008. The Scl1 protein of M6-type group A Streptococcus binds the human complement regulatory protein, factor $\mathrm{H}$, and inhibits the alternative pathway of complement. Mol Microbiol 67:584-596.

13. Caswell, C. C., H. Oliver-Kozup, R. Han, E. Lukomska, and S. Lukomski. 2010. Scl1, the multifunctional adhesin of group A Streptococcus, selectively binds cellular fibronectin and laminin, and mediates pathogen internalization by human cells. FEMS Microbiol Lett 303:61-68.

14. Chhatwal, G. S., and K. T. Preissner. 2000. Extracellular matrix interactions with gram-positive pathogens, p. 78-86. In V. A. Fischetti, R. P. Novick, J. J. Ferretti, D. A. Portnoy, and J. I. Rood (ed.), Gram-positive pathogens. ASM Press, Washington, D. C.

15. Cho, K., and M. Caparon. 2005. Patterns of virulence gene expression differ between biofilm and tissue communities of Streptococcus pyogenes. Mol Microbiol 57:15451556. 
16. Connolly, K. L., A. L. Roberts, R. C. Holder, and S. D. Reid. 2011. Dispersal of Group A streptococcal biofilms by the cysteine protease SpeB leads to increased disease severity in a murine model. PLoS One 6:e18984.

17. Courtney, H. S., H. C. Chiang, J. L. Thacker, and J. B. Dale. 1999. Serum opacity factor is a major fibronectin-binding protein and a virulence determinant of $\mathrm{M}$ type 2 Streptococcus pyogenes. Mol Microbiol 32:89-98.

18. Courtney, H. S., D. L. Hasty, and J. B. Dale. 2003. Serum opacity factor (SOF) of Streptococcus pyogenes evokes antibodies that opsonize homologous and heterologous SOF-positive serotypes of group A streptococci. Infect Immun 71:5097-5103.

19. Courtney, H. S., Y. Li, W. O. Twal, and W. S. Argraves. 2009. Serum opacity factor is a streptococcal receptor for the extracellular matrix protein fibulin-1. J Biol Chem 284:12966-12971.

20. Cramer, T., Y. Yamanishi, B. E. Clausen, I. Forster, R. Pawlinski, N. Mackman, V. H. Haase, R. Jaenisch, M. Corr, V. Nizet, G. S. Firestein, H. P. Gerber, N. Ferrara, and R. S. Johnson. 2003. HIF-1 $\alpha$ is essential for myeloid cell-mediated inflammation. Cell 112:645-657.

21. Cue, D., H. Lam, and P. P. Cleary. 2001. Genetic dissection of the Streptococcus pyogenes $\mathrm{M} 1$ protein: regions involved in fibronectin binding and intracellular invasion. Microb Pathog 31:231-242.

22. Dillon, H. C., C. W. Derrick, and M. S. Dillon. 1974. M-Antigens common to pyoderma and acute glomerulonephritis. J Infect Dis 130:257-267.

23. Dillon, H. C., and L. W. Wannamaker. 1971. Skin infections and acute glomerulonephritis, report of a symposium. Military Medicine 136:122-127.

24. Ensenberger, M. G., B. R. Tomasini-Johansson, J. Sottile, V. Ozeri, E. Hanski, and D. F. Mosher. 2001. Specific interactions between F1 adhesin of Streptococcus pyogenes and N-terminal modules of fibronectin. J Biol Chem 276:35606-35613.

25. Ffrench-Constant, C., L. Van de Water, H. F. Dvorak, and R. O. Hynes. 1989. Reappearance of an embryonic pattern of fibronectin splicing during wound healing in the adult rat. J Cell Biol 109:903-914.

26. Gao, Y., C. Liang, R. Zhao, S. Lukomski, and R. Han. 2010. The Scl1 of M41-type group A Streptococcus binds the high-density lipoprotein. FEMS Microbiol Lett 309:5561.

27. Green, N., S. Zhang, S. Porcella, M. Nagiec, K. Barbian, S. Beres, R. Lefebvre, and J. Musser. 2005. Genome sequence of a serotype M28 strain of group A Streptococcus: potential new insights into puerperal sepsis and bacterial disease dpecificity. J Infect Dis 192:760-770.

28. Green, N. M., S. B. Beres, E. A. Graviss, J. E. Allison, A. J. McGeer, J. VuopioVarkila, R. B. LeFebvre, and J. M. Musser. 2005. Genetic diversity among type emm28 group A Streptococcus strains causing invasive infections and pharyngitis. J Clin Microbiol 43:4083-4091.

29. Han, R., C. C. Caswell, E. Lukomska, D. R. Keene, M. Pawlowski, J. M. Bujnicki, J. K. Kim, and S. Lukomski. 2006. Binding of the low-density lipoprotein by streptococcal collagen-like protein Scl1 of Streptococcus pyogenes. Mol Microbiol 61:351-367.

30. Han, R., A. Zwiefka, C. C. Caswell, Y. Xu, D. R. Keene, E. Lukomska, Z. Zhao, M. Höök, and S. Lukomski. 2006. Assessment of prokaryotic collagen-like sequences derived from streptococcal Scl1 and Scl2 proteins as a source of recombinant GXY polymers. Appl Microbiol Biotechnol 72:109-115.

31. Hanski, E., and M. Caparon. 1992. Protein F, a fibronectin-binding protein, is an adhesin of the group A Streptococcus Streptococcus pyogenes. PNAS 89:6172-6176. 
32. Henderson, B., S. Nair, J. Pallas, and M. A. Williams. 2011. Fibronectin: a multidomain host adhesin targeted by bacterial fibronectin-binding proteins. FEMS Microbiol Rev 35:147-200.

33. Holbrook, K. A., and L. T. Smith. 2002. Morphology and Chemical Composition of Connective Tissue: Structure of the Skin and Tendon, p. 19-39. In P. M. Royce and B. Steinmann (ed.), Connective Tissue and Its Heritable Disorders, 2nd ed. Wiley-Liss, Inc., New York, N. Y.

34. House-Pompeo, K., Y. Xu, D. Joh, P. Speziale, and M. Höök. 1996. Conformational changes in the fibronectin binding MSCRAMMs are induced by ligand binding. $\mathrm{J}$ Biol Chem 271:1379-1384.

35. Humphries, J. D., A. Byron, and M. J. Humphries. 2006. Integrin ligands at a glance. J Cell Sci 119:3901-3903.

36. Humtsoe, J. O., J. K. Kim, Y. Xu, D. R. Keene, M. Höök, S. Lukomski, and K. K. Wary. 2005. A streptococcal collagen-like protein interacts with the $\alpha_{2} \beta_{1}$ integrin and induces intracellular signaling. J. Biol. Chem. 280:13848-13857.

37. Jaffe, J., S. Natanson-Yaron, M. G. Caparon, and E. Hanski. 1996. Protein F2, a novel fibronectin-binding protein from Streptococcus pyogenes, possesses two domains. Mol Microbiol 21:373-384.

38. Jagnow, J., and S. Clegg. 2003. Klebsiella pneumoniae MrkD-mediated biofilm formation on extracellular matrix- and collagen-coated surfaces. Microbiology 149:23972405.

39. Katerov, V., P. E. Lindgren, A. A. Totolian, and C. Schalen. 2000. Streptococcal opacity factor: a family of bifunctional proteins with lipoproteinase and fibronectinbinding activities. Curr Microbiol 40:149-156.

40. Kim, J. H., J. Singvall, U. Schwarz-Linek, B. J. Johnson, J. R. Potts, and M. Höök. 2004. BBK32, a fibronectin binding MSCRAMM from Borrelia burgdorferi, contains a disordered region that undergoes a conformational change on ligand binding. J Biol Chem 279:41706-41714.

41. Kimura, K. R., M. Nakata, T. Sumitomo, B. Kreikemeyer, A. Podbielski, Y. Terao, and S. Kawabata. 2012. Involvement of T6 pili in biofilm formation by serotype M6 Streptococcus pyogenes. J Bacteriol 194:804-812.

42. Kohan, M., A. F. Muro, E. S. White, and N. Berkman. 2010. EDA-containing cellular fibronectin induces fibroblast differentiation through binding to $\alpha_{4} \beta_{7}$ integrin receptor and MAPK/Erk 1/2-dependent signaling. FASEB J 24:4503-4512.

43. Kreikemeyer, B., M. Klenk, and A. Podbielski. 2004. The intracellular status of Streptococcus pyogenes: role of extracellular matrix-binding proteins and their regulation. Int J Med Microbiol 294:177-188.

44. Kreikemeyer, B., S. R. Talay, and G. S. Chhatwal. 1995. Characterization of a novel fibronectin-binding surface protein in group A streptococci. Mol Microbiol 17:137-145.

45. Lembke, C., A. Podbielski, C. Hidalgo-Grass, L. Jonas, E. Hanski, and B. Kreikemeyer. 2006. Characterization of biofilm formation by clinically relevant serotypes of group A streptococci. Appl Environ Microbiol 72:2864-2875.

46. Liao, Y. F., P. J. Gotwals, V. E. Koteliansky, D. Sheppard, and L. Van De Water. 2002. The EIIIA segment of fibronectin is a ligand for integrins $\alpha_{9} \beta_{1}$ and $\alpha_{4} \beta_{1}$ providing a novel mechanism for regulating cell adhesion by alternative splicing. $J$ Biol Chem 277:14467-14474.

47. Liao, Y. F., K. G. Wieder, J. M. Classen, and L. Van De Water. 1999. Identification of two amino acids within the EIIIA (ED-A) segment of fibronectin constituting the epitope for two function-blocking monoclonal antibodies. J Biol Chem 274:1787617884. 
48. Lukomski, S., N. P. Hoe, I. Abdi, J. Rurangirwa, P. Kordari, M. Liu, D. Shu-Jun, G. G. Adams, and J. M. Musser. 2000. Nonpolar inactivation of the hypervariable streptococcal inhibitor of complement gene (sic) in serotype M1 Streptococcus pyogenes significantly decreases mouse mucosal colonization. Infection and Immunity 68:535-542.

49. Lukomski, S., K. Nakashima, I. Abdi, V. J. Cipriano, R. M. Ireland, S. D. Reid, G. G. Adams, and J. M. Musser. 2000. Identification and characterization of the scl gene encoding a group A Streptococcus extracellular protein virulence factor with similarity to human collagen. Infect Immun 68:6542-6553.

50. Lukomski, S., K. Nakashima, I. Abdi, V. J. Cipriano, B. J. Shelvin, E. A. Graviss, and J. M. Musser. 2001. Identification and characterization of a second extracellular collagen-like protein made by group A Streptococcus: control of production at the level of translation. Infect Immun 69:1729-1738.

51. Maddocks, S. E., C. J. Wright, A. H. Nobbs, J. L. Brittan, L. Franklin, N. Stromberg, A. Kadioglu, M. A. Jepson, and H. F. Jenkinson. 2011. Streptococcus pyogenes antigen I/II-family polypeptide AspA shows differential ligand-binding properties and mediates biofilm formation. Mol Microbiol 81:1034-1049.

52. Manetti, A., C. Zingaretti, F. Falugi, S. Capo, M. Bombaci, F. Bagnoli, G. Gambellini, G. Bensi, M. Mora, A. Edwards, J. Musser, E. Graviss, J. Telford, G. Grandi, and I. Margarit. 2007. Streptococcus pyogenes pili promote pharyngeal cell adhesion and biofilm formation. Mol Microbiol 64:968-983.

53. Mao, Y., and J. E. Schwarzbauer. 2005. Fibronectin fibrillogenesis, a cell-mediated matrix assembly process. Matrix Biol 24:389-399.

54. Margarit, I., S. Bonacci, G. Pietrocola, S. Rindi, C. Ghezzo, M. Bombaci, V. NardiDei, R. Grifantini, P. Speziale, and G. Grandi. 2009. Capturing host-pathogen interactions by protein microarrays: identification of novel streptococcal proteins binding to human fibronectin, fibrinogen, and C4BP. Faseb J:000-000.

55. Marjenberg, Z. R., I. R. Ellis, R. M. Hagan, S. Prabhakaran, M. Höök, S. R. Talay, J. R. Potts, D. Staunton, and U. Schwarz-Linek. 2011. Cooperative binding and activation of fibronectin by a bacterial surface protein. J Biol Chem 286:1884-1894.

56. Martin, P. 1997. Wound healing-aiming for perfect skin regeneration. Science 276:7581.

57. Mohs, A., T. Silva, T. Yoshida, R. Amin, S. Lukomski, M. Inouye, and B. Brodsky. 2007. Mechanism of stabilization of a bacterial collagen triple helix in the absence of hydroxyproline. J Biol Chem 282:29757-29765.

58. O'Loughlin, R., A. Roberson, P. Cieslak, R. Lynfield, K. Gershman, A. Craig, B. Albanese, M. Farley, N. Barrett, N. Spina, B. Beall, L. Harrison, A. Reingold, C. Van Beneden, and 2007. The epidemiology of invasive group A streptococcal infection and potential vaccine implications: United States, 2000-2004. Clin Infect Dis 45:853862.

59. Oehmcke, S., A. Podbielski, and B. Kreikemeyer. 2004. Function of the FibronectinBinding Serum Opacity Factor of Streptococcus pyogenes in Adherence to Epithelial Cells. Infect Immun 72:4302-4308.

60. Oliver-Kozup, H. A., M. Elliott, B. A. Bachert, K. H. Martin, S. D. Reid, D. E. Schwegler-Berry, B. J. Green, and S. Lukomski. 2011. The streptococcal collagen-like protein-1 (Scl1) is a significant determinant for biofilm formation by group A Streptococcus. BMC Microbiol 11:262.

61. Ozeri, V., I. Rosenshine, D. F. Mosher, R. Fassler, and E. Hanski. 1998. Roles of integrins and fibronectin in the entry of Streptococcus pyogenes into cells via protein F1. Mol Microbiol 30:625-637.

62. Pahlman, L. I., P. F. Marx, M. Morgelin, S. Lukomski, J. C. M. Meijers, and H. Herwald. 2007. Thrombin-activatable fibrinolysis inhibitor binds to Streptococcus 
pyogenes by interacting with collagen-like proteins A and B. J Biol Chem 282:2487324881.

63. Palmer, E. L., C. Ruegg, R. Ferrando, R. Pytela, and D. Sheppard. 1993. Sequence and tissue distribution of the integrin $\alpha 9$ subunit, a novel partner of $\beta 1$ that is widely distributed in epithelia and muscle. J Cell Biol 123:1289-1297.

64. Pankov, R., and K. M. Yamada. 2002. Fibronectin at a glance. J Cell Sci 115:38613863.

65. Patti, J. M., B. L. Allen, M. J. McGavin, and M. Höök. 1994. MSCRAMM-mediated adherence of microorganisms to host tissues. Annual Review Microbiol 48:585-617.

66. Rasmussen, M., and L. Björck. 2001. Unique regulation of SclB-a novel collagen-like surface protein of Streptococcus pyogenes. Infect Immun 40:1427-1438.

67. Rasmussen, M., A. Eden, and L. Björck. 2000. SclA, a novel collagen-like surface protein of Streptococcus pyogenes. Infect Immun 68:6370-6377.

68. Reuter, M., C. C. Caswell, S. Lukomski, and P. F. Zipfel. 2010. Binding of the human complement regulators CFHR1 and factor $\mathrm{H}$ by streptococcal collagen-like protein 1 (Scl1) via their conserved $\mathrm{C}$ termini allows control of the complement cascade at multiple levels. J Biol Chem 285:38473-38485.

69. Roberts, A. L., K. L. Connolly, C. D. Doern, R. C. Holder, and S. D. Reid. 2010. Loss of the group A Streptococcus regulator Srv decreases biofilm formation in vivo in an otitis media model of infection. Infect Immun 78:4800-4808.

70. Rocha, C. L., and V. A. Fischetti. 1999. Identification and characterization of a novel fibronectin-binding protein on the surface of group A streptococci. Infect Immun 67:2720-2728.

71. Sakai, T., K. J. Johnson, M. Murozono, K. Sakai, M. A. Magnuson, T. Wieloch, T. Cronberg, A. Isshiki, H. P. Erickson, and R. Fassler. 2001. Plasma fibronectin supports neuronal survival and reduces brain injury following transient focal cerebral ischemia but is not essential for skin-wound healing and hemostasis. Nat Med 7:324-330.

72. Schierle, C. F., M. De la Garza, T. A. Mustoe, and R. D. Galiano. 2009. Staphylococcal biofilms impair wound healing by delaying reepithelialization in a murine cutaneous wound model. Wound Repair Regen 17:354-359.

73. Schmidt, K. H., K. Mann, J. Cooney, and W. Kohler. 1993. Multiple binding of type 3 streptococcal M protein to human fibrinogen, albumin and fibronectin. FEMS Immunol Med Microbiol 7:135-143.

74. Serini, G., M. L. Bochaton-Piallat, P. Ropraz, A. Geinoz, L. Borsi, L. Zardi, and G. Gabbiani. 1998. The fibronectin domain ED-A is crucial for myofibroblastic phenotype induction by transforming growth factor-beta1. J Cell Biol 142:873-881.

75. Shinde, A. V., C. Bystroff, C. Wang, M. G. Vogelezang, P. A. Vincent, R. O. Hynes, and L. Van De Water. 2008. Identification of the peptide sequences within the EIIIA (EDA) segment of fibronectin that mediate integrin alpha9beta1-dependent cellular activities. J Biol Chem 283:2858-2870.

76. Singh, P., C. Chen, S. Pal-Ghosh, M. A. Stepp, D. Sheppard, and L. Van De Water. 2009. Loss of integrin alpha9beta1 results in defects in proliferation, causing poor reepithelialization during cutaneous wound healing. J Invest Dermatol 129:217-228.

77. Singh, P., C. L. Reimer, J. H. Peters, M. A. Stepp, R. O. Hynes, and L. Van De Water. 2004. The spatial and temporal expression patterns of integrin alpha9beta1 and one of its ligands, the EIIIA segment of fibronectin, in cutaneous wound healing. J Invest Dermatol 123:1176-1181.

78. Sumby, P., S. Porcella, A. Madrigal, K. Barbian, K. Virtaneva, S. Ricklefs, D. Sturdevant, M. Graham, J. Vuopio-Varkila, N. Hoe, and J. Musser. 2005. Evolutionary origin and emergence of a highly successful clone of serotype M1 group A 
Streptococcus involved multiple horizontal gene transfer events. J Infect Dis 192:771782.

79. Talay, S. R., P. Valentin-Weigand, K. N. Timmis, and G. S. Chhatwal. 1994. Domain structure and conserved epitopes of Sfb protein, the fibronectin-binding adhesin of Streptococcus pyogenes. Molecular Microbiology 13:531-539.

80. Taooka, Y., J. Chen, T. Yednock, and D. Sheppard. 1999. The integrin alpha9beta1 mediates adhesion to activated endothelial cells and transendothelial neutrophil migration through interaction with vascular cell adhesion molecule-1. J Cell Biol 145:413-420.

81. Tart, A., M. Walker, and J. Musser. 2007. New understanding of the group A Streptococcus pathogenesis cycle. Trends Microbiol 15: 318-325.

82. Terao, Y., S. Kawabata, E. Kunitomo, J. Murakami, I. Nakagawa, and S. Hamada. 2001. Fba, a novel fibronectin-binding protein from Streptococcus pyogenes, promotes bacterial entry into epithelial cells, and the $f b a$ gene is positively transcribed under the Mga regulator. Mol Microbiol 42:75-86.

83. Terao, Y., S. Kawabata, M. Nakata, I. Nakagawa, and S. Hamada. 2002. Molecular characterization of a novel fibronectin-binding protein of Streptococcus pyogenes strains isolated from toxic shock-like syndrome patients. J Biol Chem 277:47428-47435.

84. To, W. S., and K. S. Midwood. 2011. Plasma and cellular fibronectin: distinct and independent functions during tissue repair. Fibrogenesis Tissue Repair 4:21.

85. Tognetti, L., C. Martinelli, S. Berti, J. Hercogova, T. Lotti, F. Leoncini, and S. Moretti. 2012. Bacterial skin and soft tissue infections: review of the epidemiology, microbiology, aetiopathogenesis and treatment: A collaboration between dermatologists and infectivologists. J Eur Acad Dermatol Venereol 26: 931-941.

86. Whatmore, A. M. 2001. Streptococcus pyogenes sclB encodes a putative hypervariable surface protein with a collagen-like repetitive structure. Microbiol 147:419-429.

87. Xu, Y., D. R. Keene, J. M. Bujnicki, M. Höök, and S. Lukomski. 2002. Streptococcal Scl1 and Scl2 proteins form collagen-like triple helices. J Biol Chem 277:27312-27318. 
Figures and Figure Legends
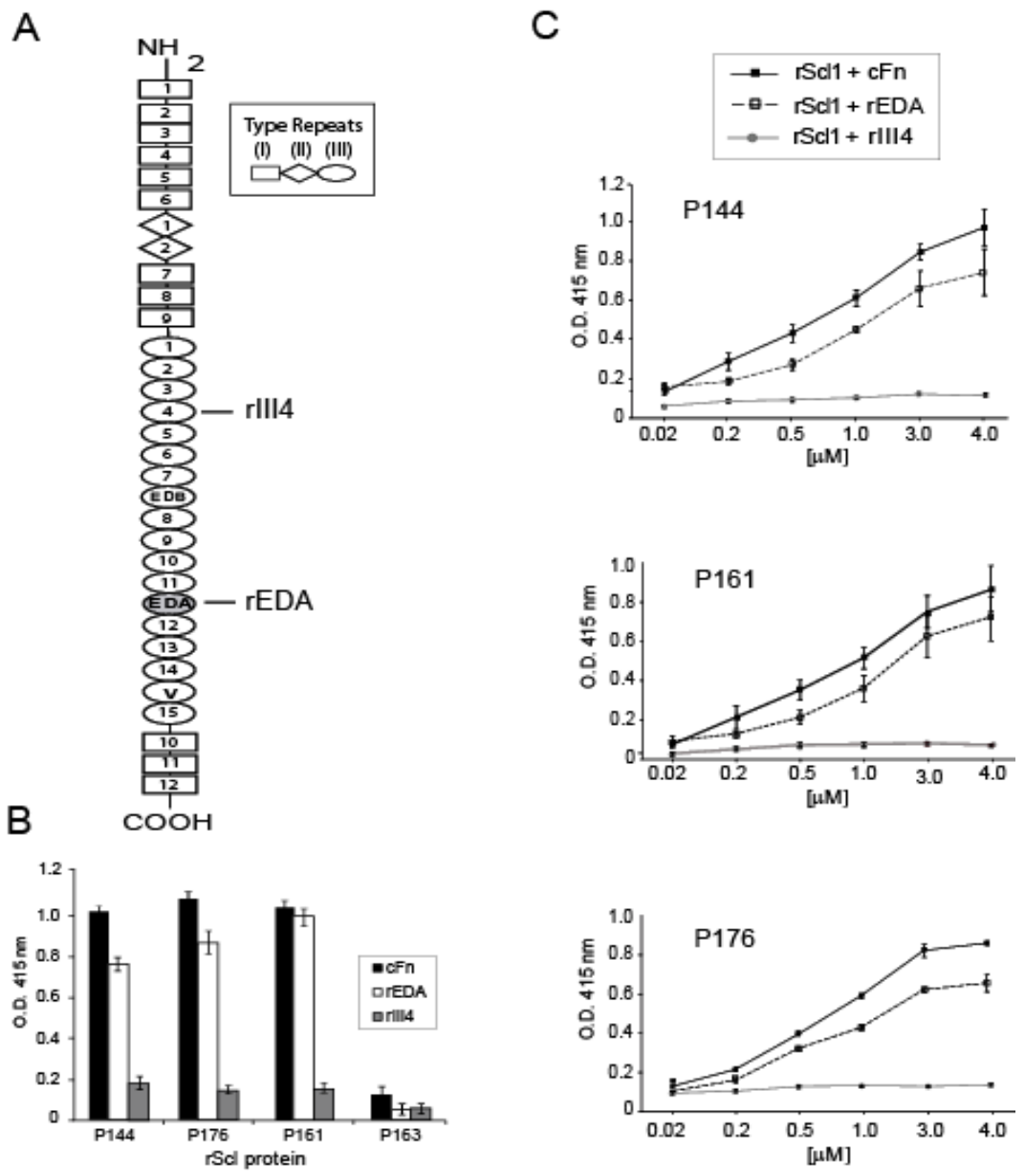

Figure 1. The EDA-domain mediates Scl1 binding to cellular fibronectin.

(A) Schematic representation of cellular fibronectin (cFn). cFn monomer depicts type I, II and III repeats. The locations of the extra domain A (EDA) and type III repeat 4 are indicated, as sources for recombinant molecules rEDA and rlll4 used. (B) Binding of ECM ligands to recombinant $\mathrm{Scl}(\mathrm{rScl})$ proteins. $\mathrm{cFn}$-binding positive recombinant Scl1 (rScl) proteins P144 (rScl1.1), P161 (rScl1.28), and P176 (rScl1.41), as well as cFn-binding negative control protein P163 (rScl2.28) were immobilized onto Strep-Tactin-coated wells and incubated with cFn or cFnderived rEDA and rIII4 polypeptides. rScl-bound ligands were detected by ELISA with ligand-specific primary antibodies and appropriate HRP-conjugated secondary antibodies. Graphic bars indicate the mean $\mathrm{OD}_{415 \mathrm{~nm}}$ normalized against BSA controls. Statistical analysis was computer from three independent experimental repeats, each performed in triplicate wells $(n=3 \pm S D)$. (C) Characterization of binding specificity between $\mathrm{rScl}$ proteins and ECM ligands. Immobilized rScl1 proteins, P144, P161, and P176, were incubated with increasing concentrations $(0.02-4.0 \mu \mathrm{M})$ of $\mathrm{cFn}$, rEDA and rlll4. Mean $\mathrm{OD}_{415 \mathrm{~nm}}$, $\mathrm{n}=3 \pm \mathrm{SD}$. 
A

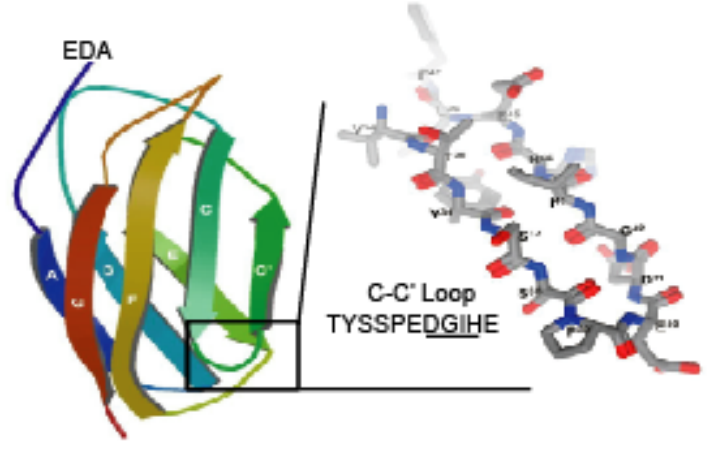

B

\begin{tabular}{cc|}
\multicolumn{2}{c|}{ Peptide Treatment } \\
$=$ No Treatment & $\square 0.1 \mathrm{mM}$ \\
$\square 0.01 \mathrm{mM}$ & $\square 1.0 \mathrm{mM}$
\end{tabular}
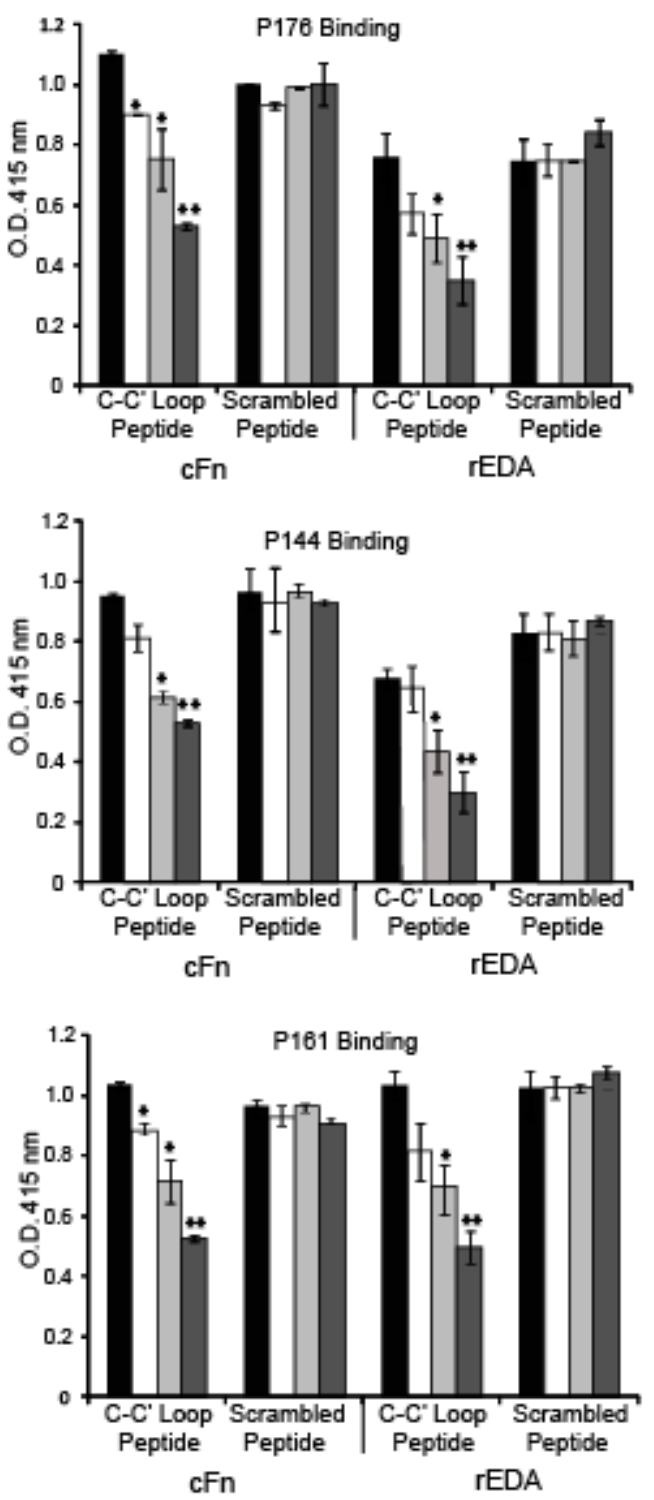

C

\begin{tabular}{|c|c|}
\hline IST-9 T & nent \\
\hline $\begin{array}{l}\text { No Treatment } \\
\square 0.1 \mathrm{\mu g}\end{array}$ & $\begin{array}{l}\square 1.0 \mu \mathrm{g} \\
10 \mu \mathrm{g}\end{array}$ \\
\hline Ш0.1 нg & \\
\hline
\end{tabular}
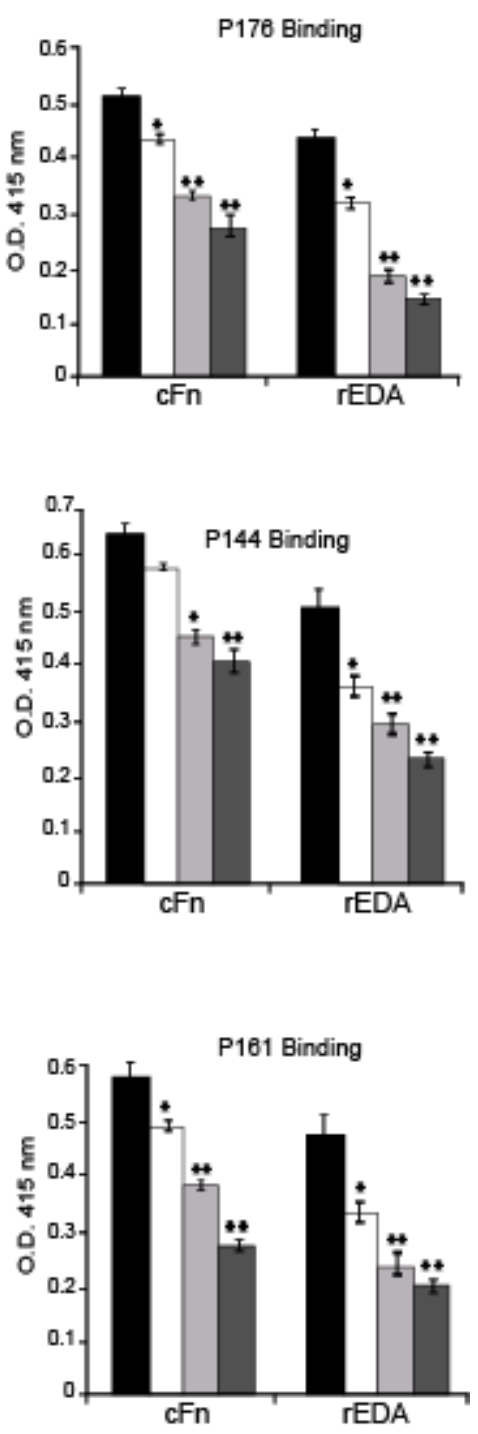
Figure 2. Scl1-cFn interaction is mediated by the C-C' loop region of EDA. Scl1-cFn interaction is mediated by the C-C' loop region of EDA. The Scl1 binding site within the EDA domain of $\mathrm{cFn}$ was mapped using peptide and antibody inhibition ELISAs. For both assays, recombinant cFn-binding positive proteins P144 (rScl1.1), P161 (rScl1.28) and P176 (rScl1.41) were immobilized onto Strep-Tactin-coated wells. Following incubation with peptide or mAb bound ECM ligands were detected with specific primary antibodies and the corresponding HRP-conjugated secondary antibodies. Graphic bars indicate the mean $\mathrm{OD}_{415 \mathrm{~nm}}$ normalized against BSA controls. Statistical analysis was computed from three independent experimental repeats, each performed in triplicate wells $(n=3 \pm \mathrm{SD})$. Statistical significance evaluates differences in ligand binding between untreated and treated samples at the levels of ${ }^{*} P \leq 0.05$, ${ }^{\star \star} P \leq$ 0.001 . (A) Structural representation of EDA. Ribbon model shows a b-sandwich composed of four b-strands, C, C', F and G, and the underlying three b-strands A, B and $E$ (adapted from Shinde et al., 2008). The stick representation of the C$C^{\prime}$ loop is shown and its 11 -amino-acid sequence is indicated. The adjacent key residues involved in $\alpha 9 \beta 1$-integrin binding (D41 and G42) and for the mAb IST-9 epitope (143 and H44) are underlined. (B) Peptide inhibition assay. Immobilized rScl1 proteins were pre-incubated with increasing concentrations $(0.0-1.0 \mathrm{mM})$ of the synthetic C-C' loop peptide or the corresponding scrambled peptide, followed by the incubation with $0.25 \mu \mathrm{M}$ ECM ligands, cFn and rEDA. ECM ligand binding to rScl1 is shown. (C) Antibody blocking assay. ECM ligands $(0.25 \mu \mathrm{M})$ were preincubated with increasing concentrations $(0.0-10 \mu \mathrm{g})$ of mAb IST-9. Mixtures were then added to rScl1-immobilized wells and allowed for rScl1-cFn/rEDA binding. 

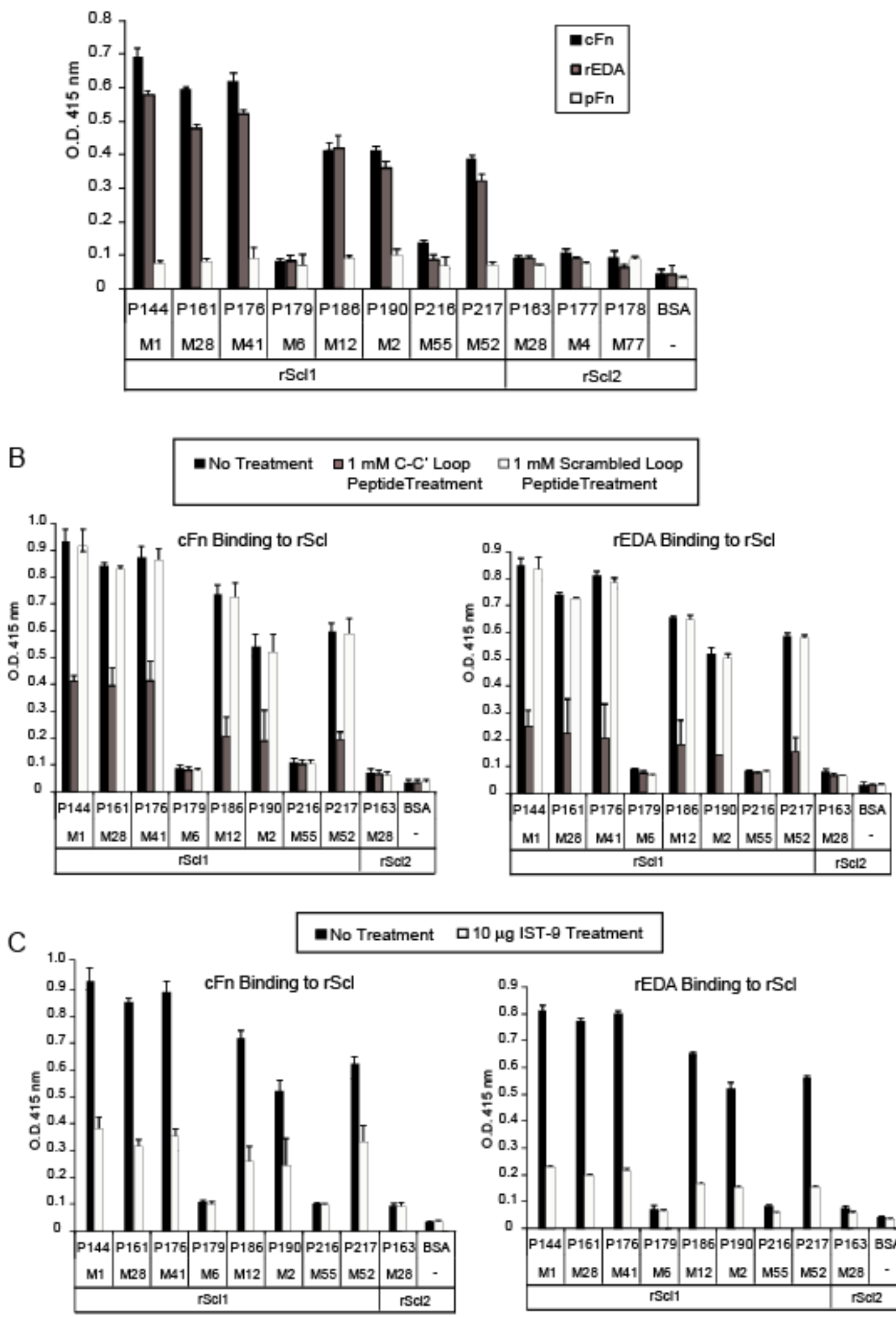

$10 \mu \mathrm{g}$ IST- 9 Treatment

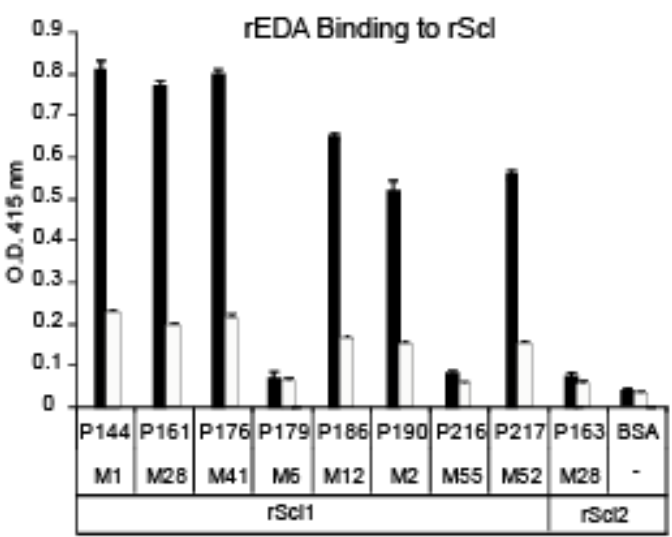


Figure 3. Scl1-EDA interaction represents a common, cFn-binding mechanism among epidemiologically diverse GAS strains.

Scl1-EDA interaction represents a common, cFn-binding mechanism among epidemiologically diverse GAS strains. Eleven $\mathrm{rScl}$ proteins derived from diverse GAS strains of various $M$ types were tested for rEDA and cFn binding. $\mathrm{rScl}$ proteins tested included the cFn-binding positive rScl1 proteins P144 (rScl1.1), P161 (rScl1.28), P186 (rScl1.12), P190 (rScl1.2), P217 (rScl1.52) and P176 ( $\mathrm{rScl1}$.41), as well as $\mathrm{cFn}$-binding negative $\mathrm{rScl} 1$ proteins $\mathrm{P} 179$ (rScl1.6) and P216 (rScl1.55), and rScl2 proteins P163 (rScl2.28), P177 (rScl2.4) and P178 $(\mathrm{rScl} 2.77)$. $r \mathrm{Scl}$ proteins were immobilized onto Strep-Tactin-coated wells and incubated with ECM ligands $\mathrm{pFn}, \mathrm{cFn}$ and $\mathrm{rEDA}$. rScl-bound ECM ligands were detected by ELISA with ligand-specific primary antibodies and the appropriate HRP-conjugated secondary antibodies. Graphic bars indicate the mean $\mathrm{OD}_{415 \mathrm{~nm}}$ normalized against BSA controls. Statistical analysis was computed from three independent experimental repeats, each performed in triplicate wells $(n=3 \pm$ $\mathrm{SD}$ ). (A) Identification of rScl1-ECM ligand binding. Immobilized $\mathrm{rScl}$ proteins were incubated with $1 \mu \mathrm{g}$ per well of each ECM ligand. Designation of each rScl1 or rScl2 construct, as well as the GAS M type from which each construct was derived, is indicated. (B) Peptide inhibition assay. Immobilized rScl proteins were pre-incubated with a $1.0 \mathrm{mM}$ concentration of the synthetic C-C' loop peptide or scrambled peptide and then with $1 \mu \mathrm{g}$ of each cFn or rEDA ligand. (C) Antibody blocking assay. ECM ligands cFn and rEDA $(1 \mu \mathrm{g})$ were pre-incubated with $10 \mu \mathrm{g}$ per sample of mAb IST-9 and then added to rScl1-immobilized wells. 
A

a $1 \mathrm{~h} \quad 24 \mathrm{~h}$
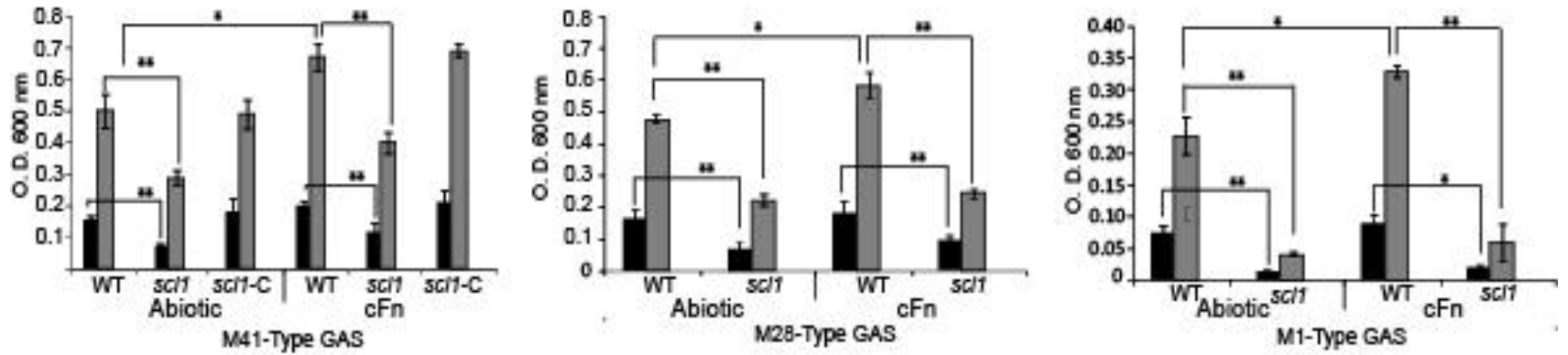

B
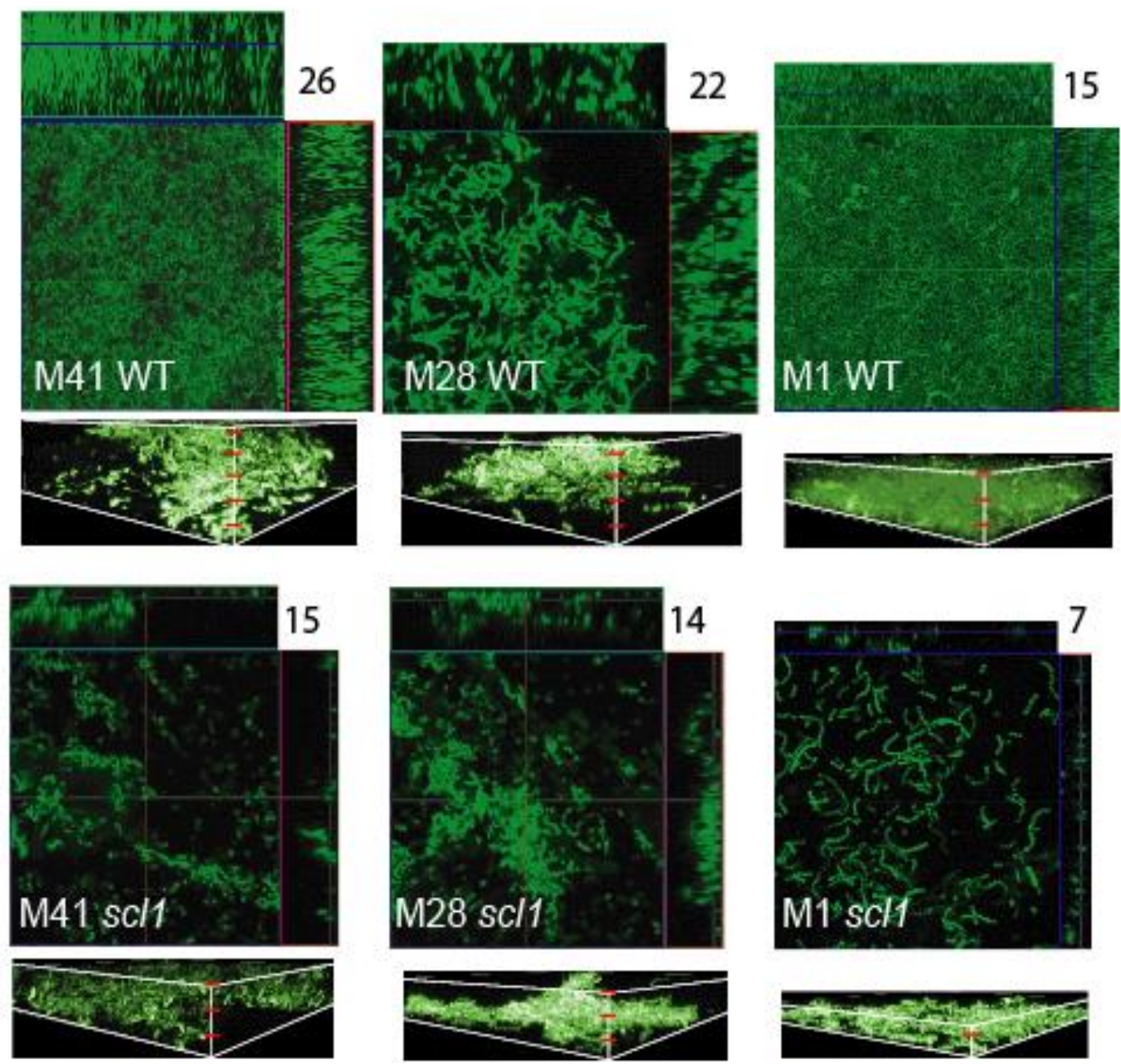
Figure 4. Scl1-cFn binding mediates and enhances biofilm formation by GAS.

WT GAS strains M41-, M28- and M1-type, their sc/1-inactivated isogenic mutants (M41scl1, M28sc/1, M1sc/1), and the M41sc/1-C mutant strain complemented intrans for Scl1.41 expression were compared for biofilm formation. (A) Biofilm formation on abiotic or cFn-coated surface. Biofilm formation for each GAS strain was evaluated spectrophotometrically following crystal violet staining. Graphic bars indicate the mean OD600 nm normalized against BSA controls. Statistical analysis was computed from three independent experimental repeats, each performed in triplicate wells $(n=3 \pm \mathrm{SD}) ;{ }^{*} P \leq 0.05,{ }^{* \star} P \leq 0.001$. (B) Analysis of biofilms formed on cFn-coated surface using confocal laser scanning microscopy. GAS strains were transformed with the GFP-encoding plasmid and grown on cFn-coated glass coverslips for $24 \mathrm{~h}$. Two-dimensional orthogonal views of GAS biofilms are representative of Z-stacks from 10 fields within a single experiment. Average vertical thickness is indicated in micrometers. Conversion to three-dimensional images was performed with conventional Zstacks, deconvoluted stepwise using AutoQuant, and transformed using NISelement software. Nick marks represent $5 \mathrm{~mm}$ in thickness and averages were taken from 10 fields within a single experiment. 
A
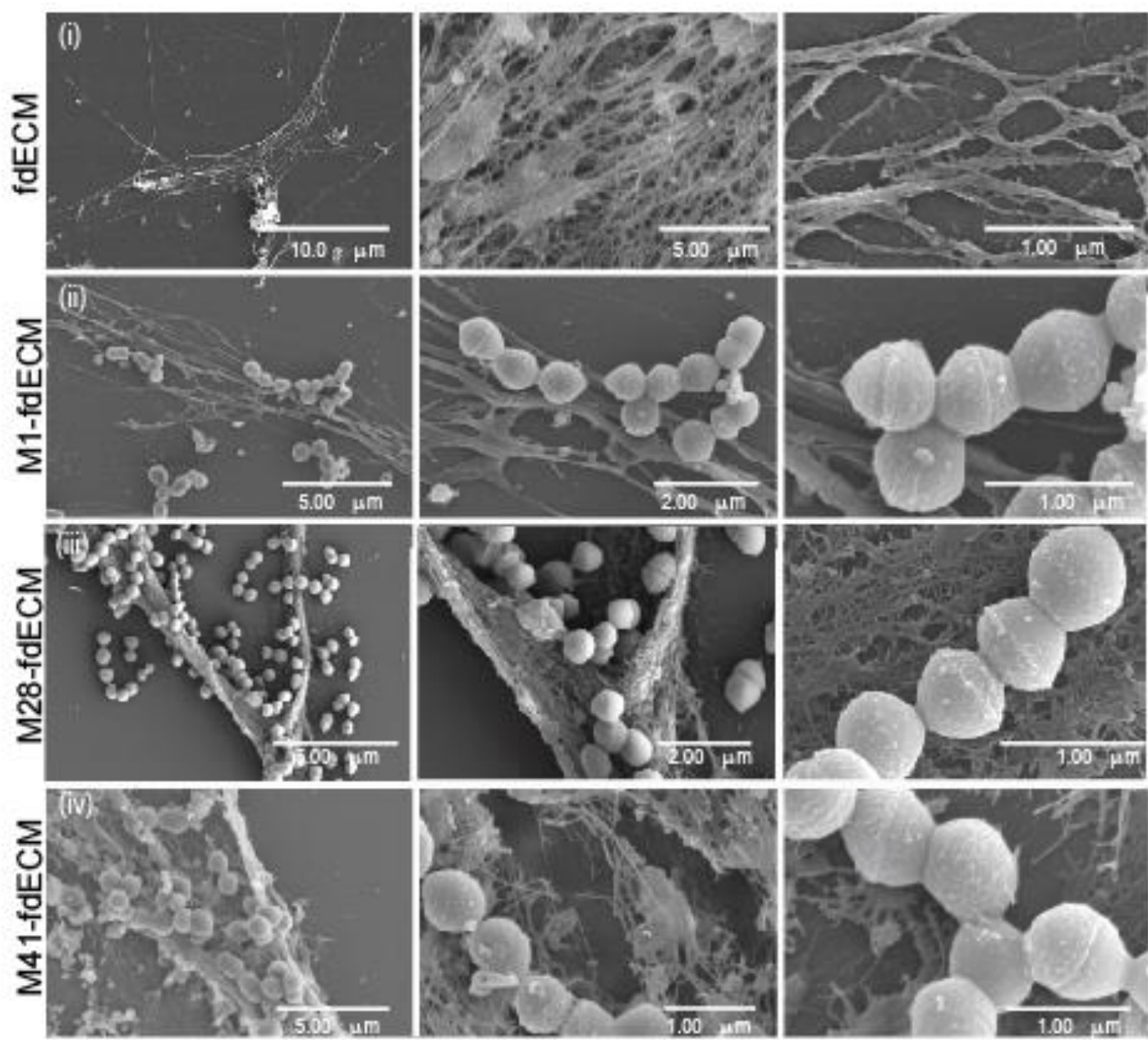

B
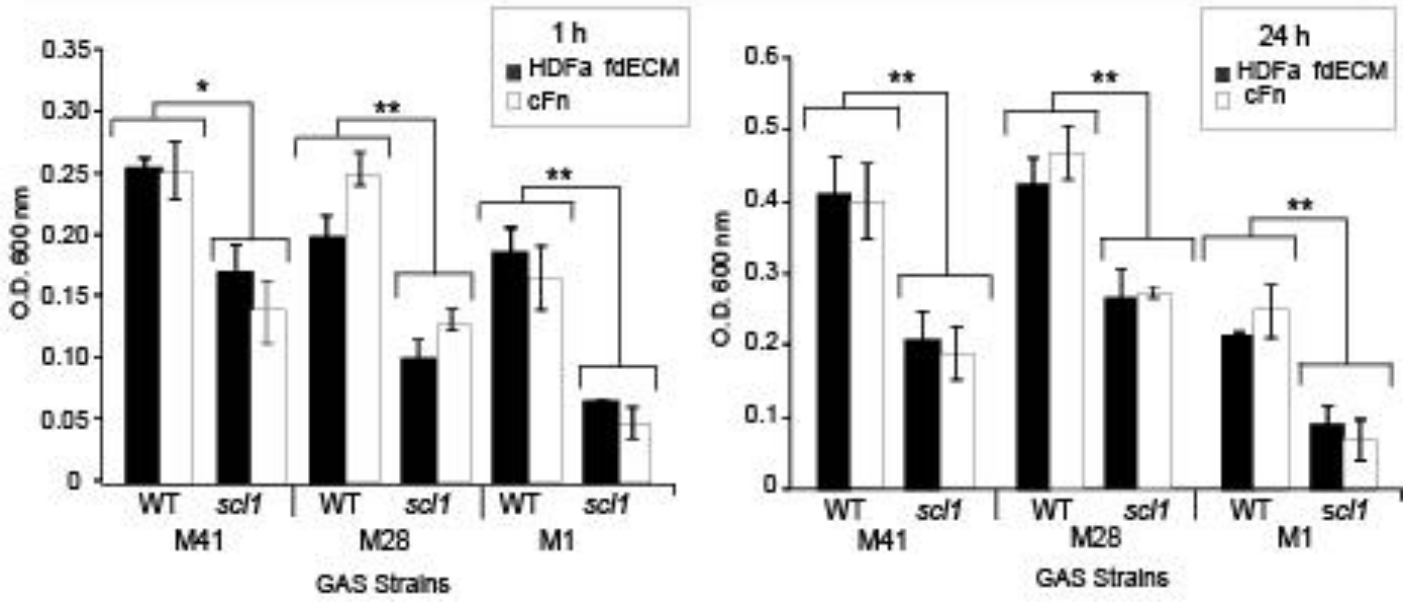
Figure 5. Scl1 binding to ECM derived from human dermal fibroblasts (HDFa) mediates and enhances biofilm formation by GAS. Adult normal HDFa were grown to confluency, detached by EGTA treatment and removed from the wells. GAS adherence to fibroblast-deposited ECM (fdECM) was analyzed using field emission scanning electron microscopy (FESEM) and overall biofilm formation on fdECM and CFn were compared spectrophotometrically following crystal violet staining. (A) Adherence of WT GAS to fdECM by FESEM. GAS strains were grown for $2 \mathrm{~h}$ on HDFa-deposited fdECM to analyze the early adherence stage in biofilm formation. FESEM images were taken with increasing magnification (left to right). Scale bars are indicated in micrometers. Row (i): images depict acellular fibrous network of deposited fibroblast-derived matrices. Rows (ii-iv): adherence of M1- (ii), M28- (iii) and M41-type (iv) GAS strains to deposited fdECM is observed. (B) fdECM supports Scl1-mediated GAS biofilm formation. WT M41-, M28- and M1-type GAS strains and their sc/1-inactivated isogenic mutants were tested for initial adherence $(1 \mathrm{~h})$ and mature biofilm $(24 \mathrm{~h})$ in wells primed with either fdECM or with commercial cFn. Graphic bars indicate the mean $\mathrm{OD}_{600 \mathrm{~nm}}$ normalized against BSA controls. Statistical analysis was computed from three independent experimental repeats, each performed in triplicate wells $(n=3 \pm$ $\mathrm{SD}) ;{ }^{*} P \leq 0.05,{ }^{*} P \geq \leq 0.001$. 

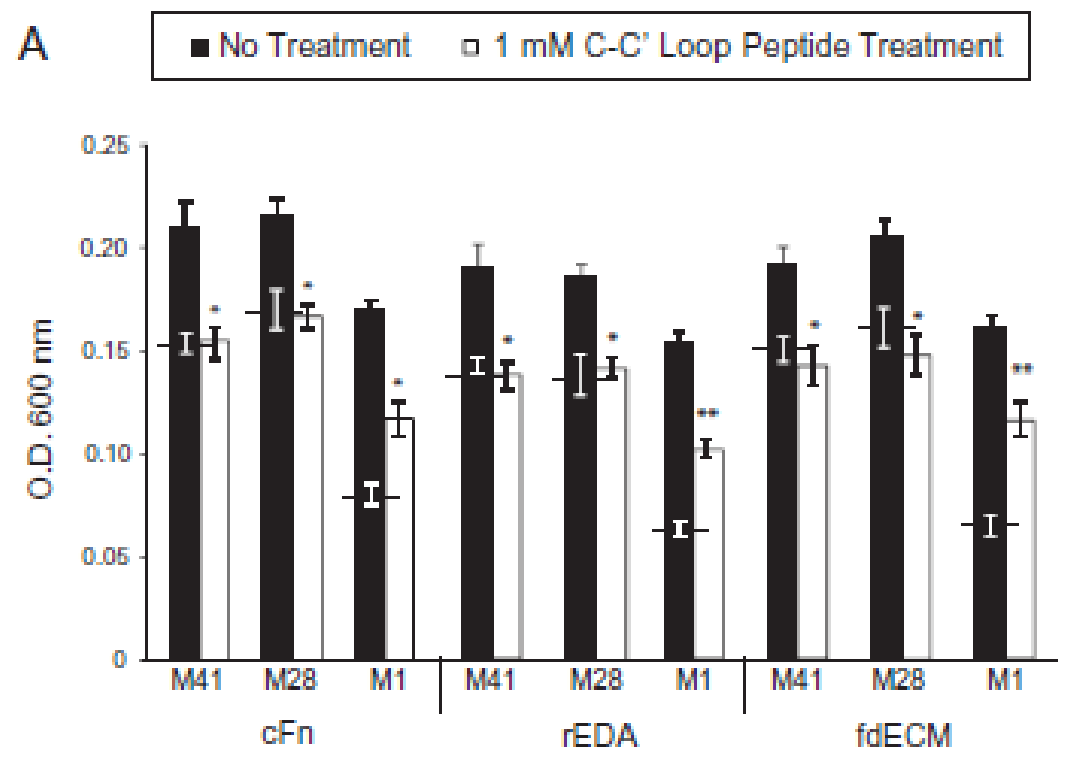

\begin{tabular}{c|ccccccccc}
\multicolumn{8}{c}{ Inhibition of Adherence } \\
\cline { 4 - 8 } C-C' Pep & $27 \%$ & $25 \%$ & $33 \%$ & $27 \%$ & $25 \%$ & $33 \%$ & $26 \%$ & $27 \%$ & $25 \%$ \\
\hline Scl1 & $28 \%$ & $24 \%$ & $51 \%$ & $27 \%$ & $26 \%$ & $54 \%$ & $24 \%$ & $24 \%$ & $54 \%$
\end{tabular}

B

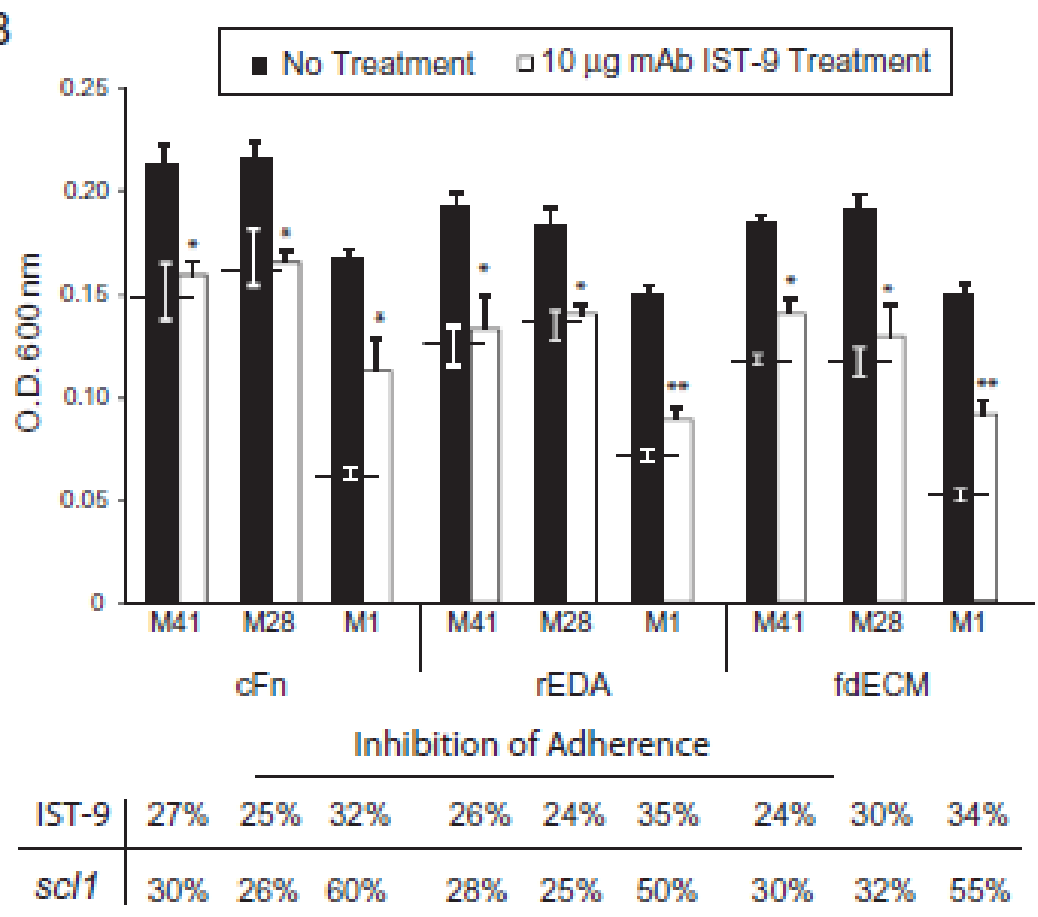


Figure. 6. Inhibition of GAS adhesion by targeting the C-C' loop of EDA.

Spectrophotometric analysis following crystal violet staining at $1 \mathrm{~h}$ is shown. Black solid horizontal lines over-imposed on bars showing untreated samples of GAS WT strains represent adhesion values of the corresponding untreated sc/1inactivated mutant strains (shown in Fig. S2). Absorbance values $\left(\mathrm{OD}_{600 \mathrm{~nm}}\right)$ reported are averages of at least two experiments done in triplicate wells. Percentage values each were compiled and shown below the graph. Statistical significance is denoted as ${ }^{*} P \leq 0.05$ and ${ }^{* *} P \leq 0.001$. (A) Inhibition of GAS adhesion with synthetic C-C' loop peptide. M1-, M28-, and M41-WT GAS strains were pre-treated with the exogenous $C^{-} C^{\prime}$ loop peptide or a scrambled loop peptide (not shown) and then added to ECM-coated wells. (B) Inhibition of GAS adhesion by mAb IST-9. ECM-coated wells were pre-treated with mAb IST-9 followed by the addition of GAS strains. Below the graph, we present \% adherence inhibition, resulting from genetic inactivation of their corresponding untreated scl1 mutants $\left(\mathrm{OD}_{600 \mathrm{~nm}}\right.$ values are shown in Fig. S2). 


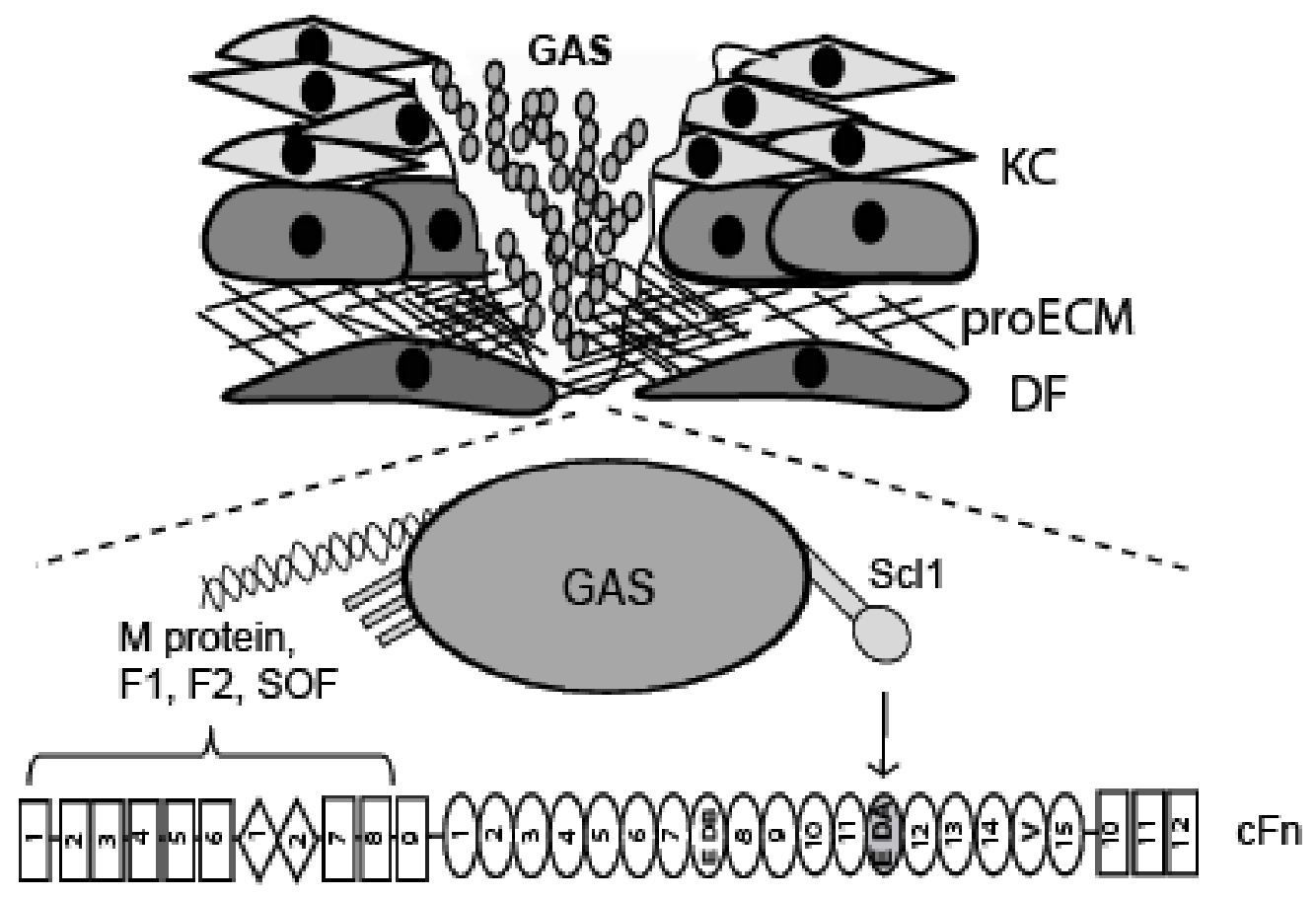

Figure 7. A model of Scl1-mediated wound colonization by GAS. (Upper schematic) Initiation of GAS infection requires a skin infringement or portal of entry. During tissue repair, host cells deposit in the wound bed a provisional ECM matrix, which includes the EDA-enriched cFn to provide scaffolding for cell migration and proliferation through integrin binding, and functions to promote the wound healing process. (Lower schematic) GAS utilizes the Scl1 protein for targeted binding to the EDA domain of cFn. This Scl1 binding to EDA/cFn variant promotes GAS attachment and colonization of the wound in concert with other GAS adhesins that bind the N-terminal region of Fn. KC, keratinocytes; proECM, provisional extracellular matrix; DF, dermal fibroblasts. 
A

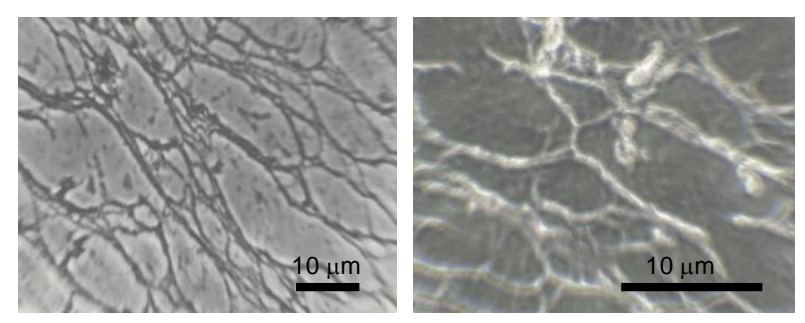

B

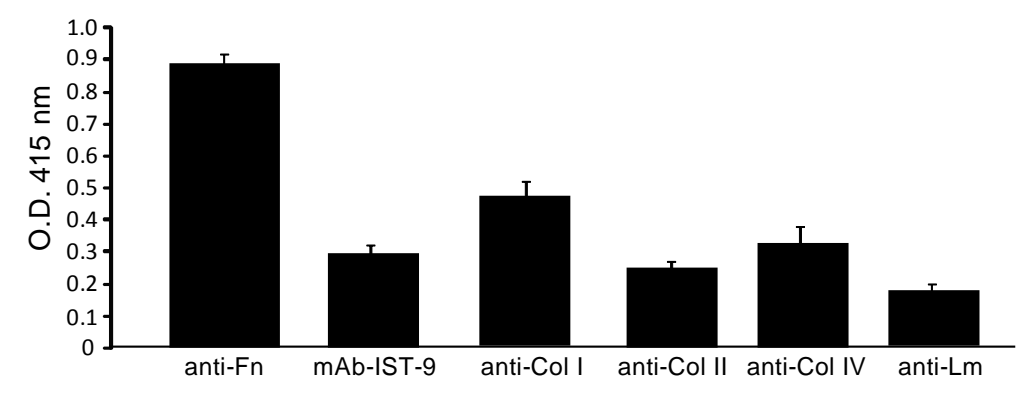

Figure S1. Characterization of fibroblast-derived extracellular matrix (fdECM). Adult human dermal fibroblasts (HDFa) were grown in Medium 106 to confluency, detached from the underlying fdECM by EGTA treatment, and removed from the wells. (A) Ponceau $S$ staining shows a complex fibrillar network of fdECM deposited by HDFa cells. (B) Composition of fdECM network. fdECM deposited in wells was evaluated for the presence of $c F n$, EDA/cFn, collagen type I, II, and IV, and laminin (shown from left to right respectively) by ELISA using specific primary antibodies and secondary HRP-conjugated antibody. Statistical analysis was computed from three independent experimental repeats, each performed in triplicate wells $(n=3 \pm S D)$. 

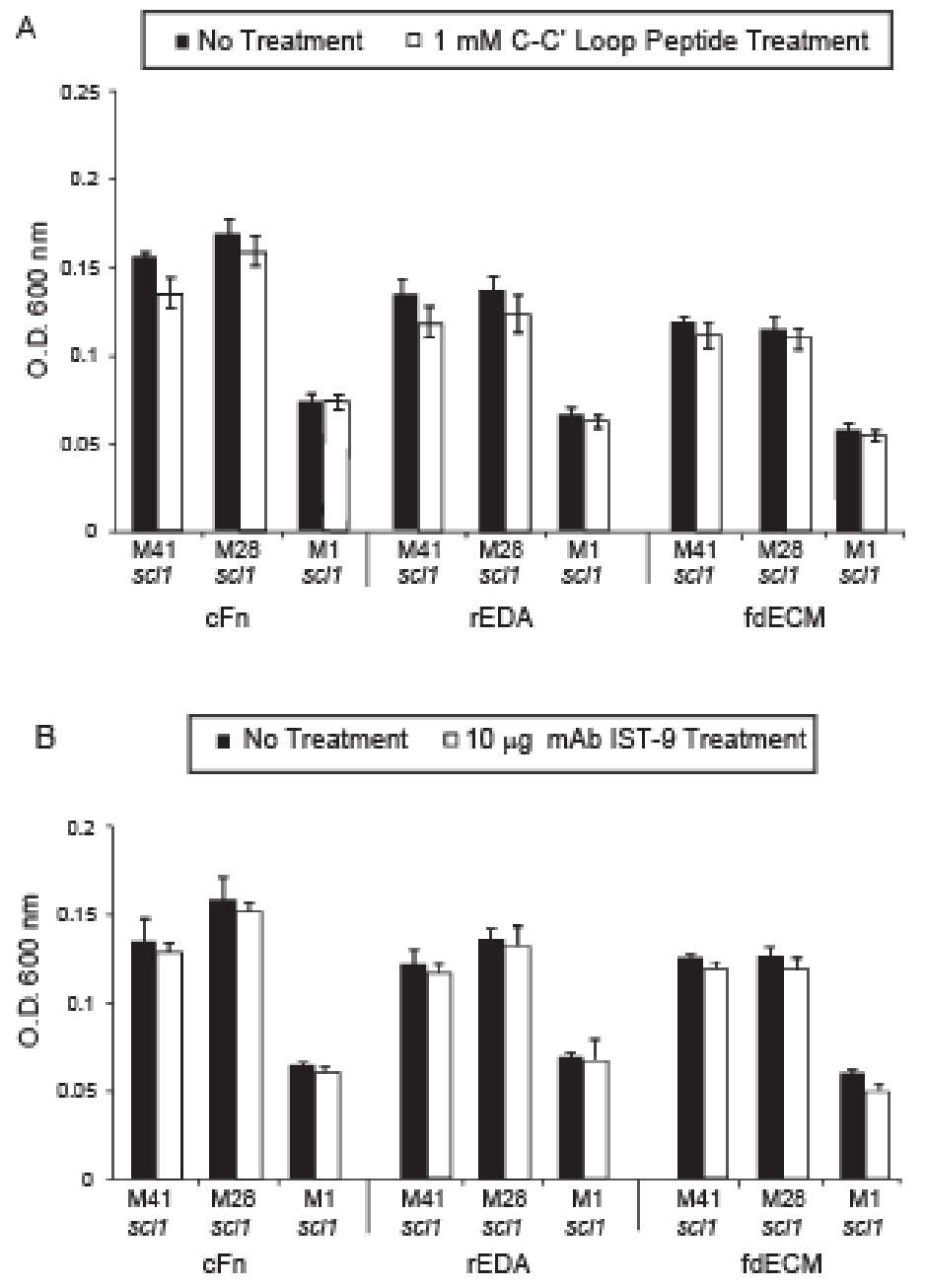

Figure S2. Adhesion inhibition of GAS sc/1 mutants by targeting the C-C' loop of EDA. Spectrophotometric analysis following crystal violet staining at $1 \mathrm{~h}$ is shown. Graphic bars indicate the mean O.D.600nm normalized against BSA controls. Statistical analysis was computed from three independent experimental repeats, each performed in triplicate wells ( $n=3 \pm S D)$. Result: No statistically significant decrease in binding to any ECM by the GAS sc/1-inactivated mutants was observed. No strains were inhibited with the treatment of the scrambled peptide (data not shown). (A) Inhibition of GAS adhesion by C-C' loop peptide. M1 scl1, M28 scl1, and M41 scl1 mutant strains were treated with synthetic C-C' loop peptide or a scrambled loop peptide (not shown) and added to ECM-coated wells. (B) Inhibition of GAS adhesion by mAb IST-9. ECM-coated wells were pretreated with mAb IST-9 followed by the addition of GAS Scl1-negative mutant strains. Result: No statistically significant decrease in binding to any ECM by the GAS sc/1-inactivated mutants was observed. 
Chapter 5:

General Discussion 
Group A Streptococcus is a globally disseminated, specialized human pathogen possessing numerous pathogenicity factors, including the Scl1 protein. Current work identified a novel mechanism by which GAS utilizes the Scl1 protein to target an extra domain A (EDA)-containing spliced variant of cellular fibronectin (cFn) (Chapter 2 \& 4) that enhances biofilm formation (Chapter 3) in vitro. Because expression and deposition of EDA/cFn in the wound plays an essential role in cicatrisation, the acquisition of the selective EDA/cFn binding by GAS, mediated by surface-attached Scl1, suggests a potential mechanism to abrogate the healing process allowing GAS to stably colonize tissue at the portal of entry and to establish biofilm within infected tissue. The implications of this mechanism rely on the importance of $\mathrm{cFn}$ in tissue repair and the impediment that infecting GAS strains may cause to this process. Therefore, the proposed Scl1-mediated wound colonization model (Chapter 4, Figure 7) will be outlined in the following sections. 
Initiation of the Wound Healing Process. The dynamics of tissue repair and regeneration are complex and reliant upon the cellular interaction with the extracellular matrix (ECM). The ECM is comprised of numerous macromolecules including collagens, fibronectins, laminins, elastin, and proteoglycans, each having a specific biological function. Specifically, the fibronectins, $\mathrm{pFn}$ and $\mathrm{cFn}$, make up a large heterogeneous group of glycoproteins that have been intensely studied and characterized. cFn is predominantly produced by macrophages, human dermal fibroblasts, and myofibroblasts that are distributed within the dermis and basement membrane of the dermis-epidermis interface. cFn has varying functions but is critically important during vertebrate development and, most relevant to the current research, in wound healing. Following skin infringement (Figure $1 \mathrm{~A}$ ), pFn is deposited within the injured site and along with fibrin and provides an initial soluble extracellular matrix. This matrix provides a base for cell migration and will function as a template for the future mature ECM. Recruited platelets release cytokines inducing an inflammatory response with macrophages and neutrophils entering the wound area. In a short time, granulation tissue is constructed as fibroblasts begin secreting collagen and $\mathrm{EDA} / \mathrm{cFn}$ in the wounded site to produce an insoluble, provisional matrix (proECM). cFn orchestrates integrin ligation, organization of the new granulation tissue, and restructuring of the ECM to restore a stable, scaffold for cellular regeneration $(28,34,70,87,98,121,129)$. Simultaneously, keratinocytes (Kn) begin to migrate towards and over the wounded site secreting laminin that interacts with integrins on the surrounding cells and form a dermoepidermal 
basement membrane forming a new epithelial layer. Kn cells also produce matrix metalloproteinases (MMPs), specifically MMP-1, that degrade unneeded native collagens to clear the wound $(28,67,114)$. Finally, contraction of the wound is initiated by myofibroblasts bridging the surrounding collagen and cFn with adjacent cells. During this process, cFn is critical for rebuilding of ECM and its biological importance is suggestive as to the reason why many microorganisms, including GAS, target and bind the fibronectins. 
Scl1-Mediated GAS Colonization of Wounded Tissue. It is suggested that microbial colonization occurs in all cutaneous and chronic wounds, regardless of whether wounds are infected endogenously or exogenously. Common features of the cutaneous acute and chronic wounds are inflammation, and disruption and destruction of the tissue. However, in case of chronic infections, the lack of progression in the wound healing process and lack of response to treatment occur and are associated with biofilm formation (36). Staphylococcus aureus and GAS are among the most commonly found in acute, subcutaneous and cutaneous wounds, as well as in chronic wounds (103). It has been demonstrated that infection of the wound with $S$. aureus causes a disruption of the normal regeneration of tissue (104). The loss of skin integrity can be caused by insect bites, cuts, burns, ulcers, dermatitis, surgeries or found in regions of ongoing infection. The temperature and $\mathrm{pH}$ of the skin, as well as, poor hygiene also contribute to tissue integrity. Sustainment of resident or transient flora colonization within the wounded tissue depends on immunological, microbial, and environmental factors (123). The introduction of GAS into a skin infringement has the potential to develop into acute or chronic infections based on the virulent nature of the strain and its capacity to adhere to host cells and establish colonization. With GAS's entrance into a wound site, the bacterium will utilize an array of surface adhesins expressed by a given strain, such as the Fn-binding proteins, referenced in Chapter 1, to cooperatively interact with host cellular and extracellular components. We have previously reported that the Scl1 protein of M41-type GAS interacts with the collagen-binding integrins, $\alpha_{2} \beta_{1}$ and $\alpha_{11} \beta_{1}$, via a 
GLPGER sequence motif located within the CL region of Scl1. It was further shown that Scl1-integrin binding facilitates GAS adherence and internalization into human epithelial and endothelial cells, which also results in an enhanced pathogen survival and re-emergence from these cells $(11,12,46)$. This is representative of one Scl1-mediated mechanism for GAS to initiate colonization. Investigations presented here identified targeted binding mediated by the $\mathrm{V}$ region of Scl1 to selected human ECM components, cFn and Lm, thus, providing a second mechanism of adherence and demonstrating a dichotomy of ligand binding by Scl1 proteins (Chapter 2) (81). The significance of the Scl1-cFn interaction characterized in our early work, subsequently led to a discovery of a new GAS-cFn-binding mechanism, which is mediated by the extra domain $A$ (EDA) of cFn (Chapter 4), with intriguing potential consequences to wound healing.

Although cFn is transcribed from a single gene, there are up to twenty isoforms identified in human tissues that are generated by tissue-specific alternative splicing of the mRNA transcript occurring among the type III repeats (44). cFn contains extra domains EDA and EDB, not found in pFn (Chapter 4, Figure 1), and has more than a dozen binding sites that recognize and bind important biological molecules and integrins, many of which bind the common RGD motif $(65,87,121)$. However, the EDA domain binds additional integrins that are different from the RGD-recognizing integrins (129). Structurally, the EDA domain is homologous to the type III repeats. Located between type III11 and III12, it forms a $\beta$-strand sandwich composed of two $\beta$-sheets containing four $\beta$ - 
strands, C-C', F-G, with three underlying $\beta$-strands $A-B$, and $E(30,60,61)$. Expression of this domain is critical during periods of embryogenesis and organogenesis. In non-diseased conditions, expression of EDA is downregulated and maintained at low levels ( $<1 \%$ total Fn in the body) $(91)$; however, during certain pathological states and specifically in wound healing, EDA expression is significantly up-regulated. Functionally, it mediates interactions with $\alpha_{4} \beta_{1}, \alpha_{9} \beta_{1}$, and $\alpha_{4} \beta_{7}$ integrins (131) to induce fibroblast differentiation $(56,107)$ and also contributes considerably to accelerate kerotinocyte migration during wound healing $(33,35,108)$. The EDA-integrin interaction is mediated by an eleven amino-acid loop sequence (TYSSPEDGIHE) located between the $\mathrm{C}$ and C' $\beta$-strands with the D-G being the targeted amino acids. In general, the binding of cFn to a ligand often results in conformational changes exposing "cryptic" binding sites or sites that are not readily accessible until the structure of the molecule is stretched or bent. The C-C' loop region of EDA is characterized as one of these sites. Several integrin-blocking monoclonal antibodies encompassing the C-C' loop region of EDA, such as mAb IST-9, were generated that inhibited the $\alpha_{4^{-}}$and $\alpha_{9}$-integrin-mediated cell adhesion $(60,61)$. Here, in a series of ELISA-based studies, it was identified that rScl1 proteins derived from M41-, M28- and M1-type GAS bound recombinant EDA (rEDA) and that binding could be blocked more than $50 \%$ by pre-treatment of rEDA with the mAb IST-9 or by pre-treatment of rScl1 proteins with the synthetic C-C' loop sequence. If this in vitro observed activity of rScl1 functions in vivo during GAS infection, cell-surface Scl1 could compete with integrins for EDA/cFN binding abrogating human cell 
migration and adhesion, thus, impairing wound healing. As shown in Figure $1 \mathrm{~B}$, implications of this data suggest that Scl1-EDA binding may affect several other cell types that express $\alpha_{9} \beta_{1}$ integrin, and participate in wound healing and inflammation, including epidermal keratinocytes $(108,109)$, as well as neutrophils, epithelial and muscle cells $(84,119)$. This hypothesis, however, has yet to be verified experimentally.

In the later stages of wound healing and in preparation of the wound bed for tissue regeneration, matrix metalloproteinases are often present. MMPs, such as MMP-1, also known as interstitial collagenase and fibroblast collagenase, degrades unneeded native collagens, specifically collagens type I and III. MMPs target open-state regions in the collagen triple-helix that lie adjacent to the MMP cleavage site $(77,102)$. Due to the structural mimicry of Scl1 to collagen, it has been questioned if human MMPs will cleavage the CL region of Scl proteins thus, eliminating the Scl1-cFn interaction. Yu and coworkers engineered chimeric rScl proteins containing fragments of the human collagen type III embedded within the CL-region of a rScl2 protein (135). They reported that the original CL domain of rScl2 was not cleaved by either MMP-1 or MMP-13, whereas both efficiently degrade triple-helical mammalian collagens. Insertion of a minimum of $5 \mathrm{GXY}$ triplets, encompassing the original MMP recognition and cleavage site, made the chimeric rScl2-huCol III protein sensitive to MMP cleavage. It will be important to test if GAS secreted proteases, such as SpeB, and or collagenases cleave Scl1 proteins at the cell surface, thus, eliminating the heterophilic Scl1-cFn interaction as well as the hemophilic Scl1-Scl1 interactions. Our earlier work showed that 
the collagen triple helices of various $\mathrm{rScl}$ constructs thermally unfold above normal human core-body temperature of $37^{\circ} \mathrm{C}(40,128)$, whereas they are stable at a human skin temperature $\sim 34^{\circ} \mathrm{C}$. These two phenomena e.g., Scl1 proteolysis and thermal unfolding may have important biological consequences for GAS wound colonization via ECM binding, for a subsequent tissue microcolony formation, and for a successful infection of the human host. This line of future studies is included in our Scl1-mediated biofilm model shown in Figure 2. 
Scl1-Mediated GAS Biofilm Formation within Wounded Tissue. Bacterial biofilms are well-documented among both acute and chronic infections (103). Biofilms denote a serious clinical problem and are present in up to $65 \%$ of human bacterial infections (92). By broad definition, biofilm is an aggregation of surfaceassociated, or sessile, microorganisms often encased within an extracellular polymeric substance also called glycocalyx $(50,103)$, which contains complex biological material containing polysaccharides, DNA, proteins, and lipids with "crypts" or water channels for nutrient flow $(22,25)$. Populations of microbes within a biofilm have an altered genotype and may undergo multiple phenotypic switches such as altered polysaccharide profiles and adhesin expression, as well as morphological changes as compared to their planktonic counterparts $(15,73$, 113). Biofilm establishment within wounds provide numerous advantages for organisms in survival, including an increase in antimicrobial resistance up to a thousand-fold (32), as well as a decreased sensitivity to immunological and chemical assault. Studies of the structural and physiological features of various biofilm models support the hypothesis that bacterial biofilms play an important role in wound colonization, infection, and abrogation of wound healing (27, 38, $76,79,80,89,90,125)$.

Over the course of the last decade, research on GAS has shown a strainto-strain variation for the capacity of biofilm formation $(59,81)$. GAS biofilm was first reported in human clinical skin specimens in the form of tissue microcolonies encased in a glycocalyx (1). GAS biofilm was also associated with previously unreported penicillin sensitivity and possible antibiotic treatment failure (18). 
Gene expression of sessile GAS cells associated with biofilm differ from their planktonic form; for example, the Scl1 expression was upregulated during initial stages, whereas the streptococcal cysteine protease, SpeB, was over-expressed within mature biofilm $(15,101)$. Studies employing GAS isogenic mutants with altered surface components, including proteins, pili, capsule and lipoteichoic acid, as well as mutants in regulatory genes and wide-spectrum secreted protease had significant impact on biofilm phenotype $(20,26,54,66)$.

An initial step in GAS colonization of a wounded site is often mediated by surface adhesins. As proposed in our Scl1-mediated biofilm model (Figure 2), planktonic GAS cells utilize the Scl1 protein to secure attachment to EDAcontaining cFn expressed by wounded cells at a site of skin injury and initiate host colonization. As colonization progresses, sessile GAS cells proliferate producing swells of microcolonies within the infected area. Over time, these microcolonies develop into macrocommunities that may remain intact forming an immune- and antimicrobial-resistant biofilm. Biofilm dispersal and bacterial dissemination may be induced by environmental factors such as elevated body temperature or loss of nutrients, as well as the production of bacterial proteases such as SpeB directly altering the structural stability of protein-protein interactions. Loosely-associated small microcolonies or even chains of GAS will eventually filter into adjacent tissues to colonize another niche ultimately repeating the process.

This body of work directly addresses the initial adherence stage of GAS biofilm formation and supports our proposed model. With the identification of the 
Scl1-EDA/cFn interaction, we proposed that this binding greatly or significantly enhances GAS biofilm formation via a bridging mechanism to host cells. To address this hypothesis, we investigated three epidemiologically different GAS strains for their capacity to form biofilm on untreated or ECM-deposited surfaces: M41, M1, and M28. The M41-type GAS strain was used as a representative strain, which has been historically associated with non-invasive skin infections. During the 1950s-60s, outbreaks of impetigo and other superficial infections occurred in regions of the United States (Minnesota and Alabama) as well as the island nation, Trinidad. These infections were linked to acute glomerulonephritis, a rare kidney infection sometimes occurring after streptococcal infections $(2,23$, 24, 124). For comparison, M1-type GAS, a highly invasive strain and the most commonly isolated from suppurative infections, and M28-type GAS, a strain historically over-represented in puerperal sepsis cases as well as invasive infections, were used. We demonstrate using a conventional crystal violet staining assay that these strains have varying capacity to form biofilm on untreated (plastic) surfaces with the M41-type producing a more robust biofilm as compared to M1 and M28. This observation was confirmed visually by microscopy and revealed distinct variations in cell-surface morphology and microcolony architecture for each strain. In assessment of wild-type strains and their sc/1-inactivated isogenic mutants in biofilm formation, we observed a significant decrease in biofilm capacity for all mutant strains indicating an important role for the Scl1 protein in this process. Directly supporting our model, we observed increased biofilm formation when GAS strains were grown on ECM- 
deposited surfaces (cFn, rEDA, or fdECM) as compared to those grown on plastic (Chapter 3). Thus, these studies reveal a novel mechanism that can be implemented by GAS during the initial stages of colonization within a skin wound that significantly increases the probability of stabile adherence to host cells facilitating the potential for biofilm formation.

Concluding Remarks. In GAS pathogenesis, the Scl1 protein has been conclusively established as an important, multifunctional surface adhesin. Being ubiquitously expressed by GAS strains and participating in the binding of biologically significant molecules such as cellular fibronectin, it is an indispensable virulence factor for host colonization among pathogenically diverse strains. With research ongoing to further dissect molecular mechanisms mediated by GAS surface components, this work provides substantial support toward this effort and for future efforts to continue the characterization of the streptococcal collagen-like proteins. 
Figures and Figure Legends

Figure 1

A
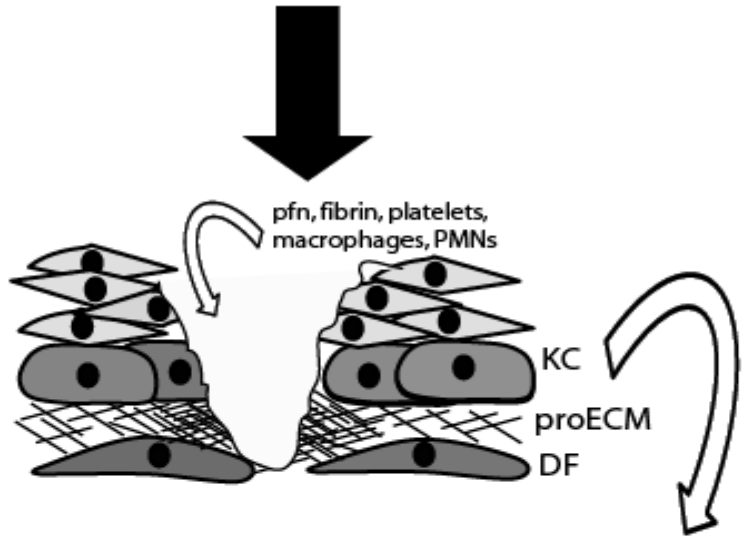

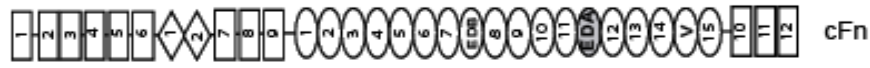

B
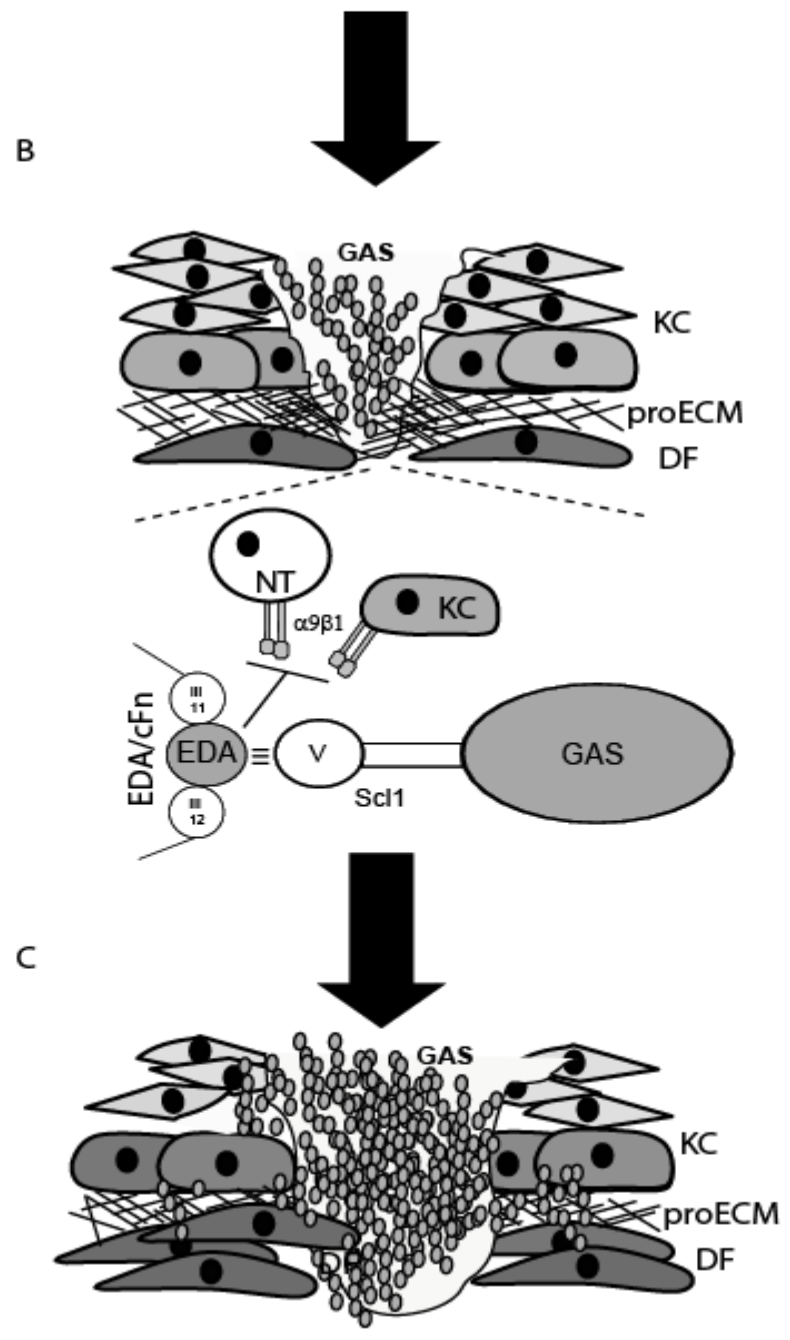
Figure 1. Step-by-step model of wound healing and role of Scl1 in host colonization.

A. Wound formation and initiation of healing process. A skin infringement occurs. Plasma fibronectin $(\mathrm{pFn})$ and fibrin are deposited within the wounded site and recruit platelets to form a clot. Platelets induce an inflammatory response by recruiting macrophages and neutrophils (PMNs) to the area, which begin to engulf and degrade necrotic cells and unneeded ECM components. Dermal fibroblasts (DF) begin secreting collagens and EDA-containing cellular fibronectin (EDA/cFn) into the site to compose a provisional ECM (proECM) that will be used as a scaffold for newly constructed ECM. Keratinocytes begin to proliferate and migrate toward and over the wound forming a monolayer to secure the site.

B. Scl1-mediated wound colonization by GAS. (Upper schematic) Initiation of GAS infection requires a skin infringement or portal of entry. (Bottom schematic; NT and KC cells are not shown according to their location in the wound) During tissue repair, multiple cells deposit in the wound bed a provisional ECM matrix, which includes the EDA-enriched cFn to provide scaffolding for cell migration and proliferation through the binding of $\alpha_{9} \beta_{1}$ integrin, and functions to promote the wound healing process. GAS utilizes the Scl1 protein for targeted binding to the EDA domain of $\mathrm{cFn}$, thus, competing with $\alpha_{9} \beta_{1}$-mediated binding of the inflammatory and epithelial cells. This competitive Scl1-EDA binding directly promotes GAS attachment and colonization of the wound which also may delaying the healing process allowing increased time to establish infection. KC, (keratinocytes); proECM (provisional extracellular matrix); DF, (dermal fibroblasts); NT, (neutrophils).

C. Scl1-mediated biofilm formation in wounded tissue. Microcolonies of GAS proliferate in the wounded site, stabilize their adherence to surrounding host cells via the Scl1-EDA/cFn "bridging" mechanism, and develop into a large microcommunity resistant to penetration of host immunity or antimicrobial attack. Individual GAS cells, chains or small microcolonies loosely associated with the community will disseminate into the tissue, adhering to surrounding host cells or ECM components to establish secondary niches that initiate the biofilm formation process again. 

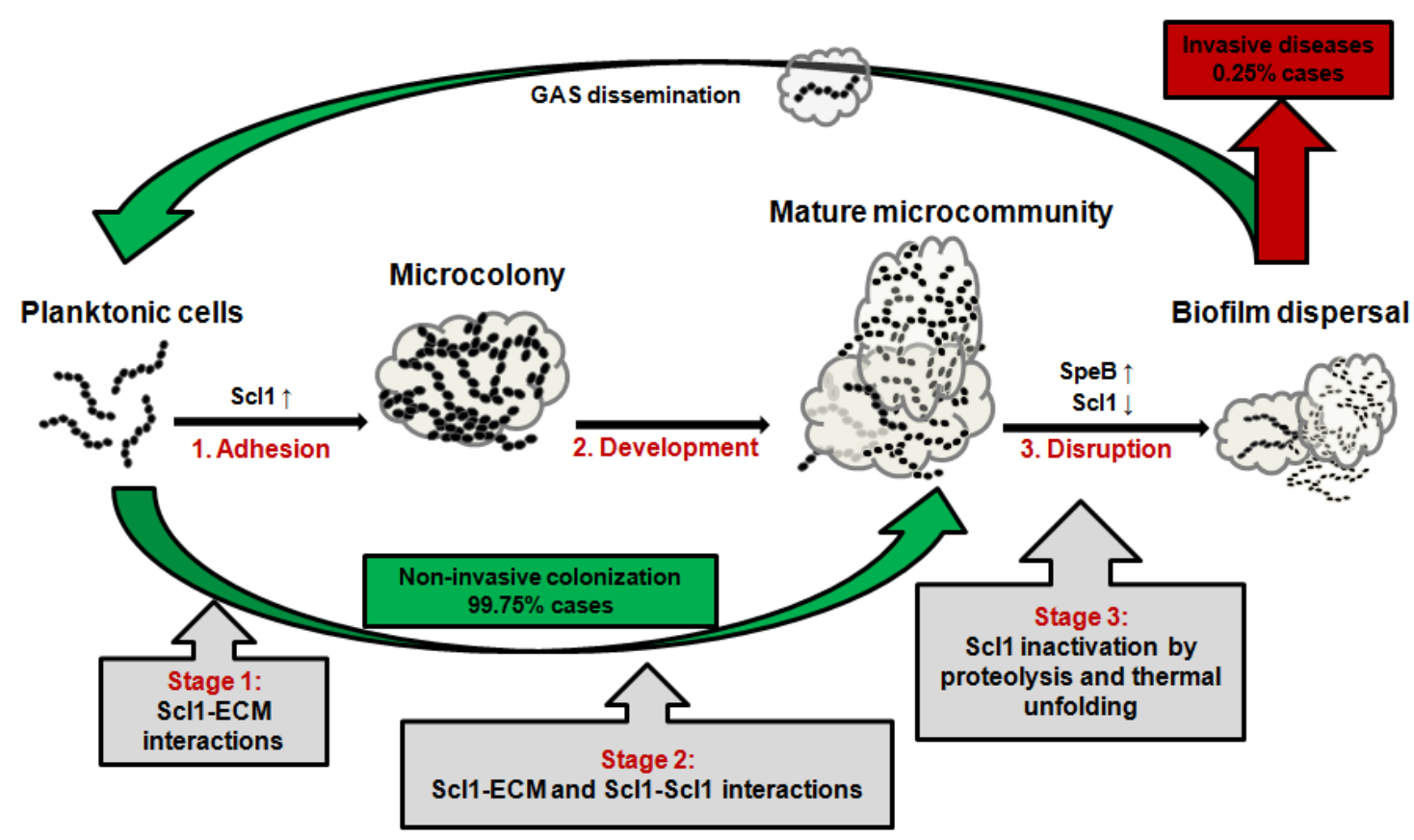

Figure 4. Scl1-mediated GAS biofilm formation model. Stage 1 of GAS biofilm formation is often a protein-protein mediated interaction to initiate binding to a substratum. Scl1 expressed by planktonic GAS cells binds extracellular matrix (ECM) components as a "bridging" mechanism to adhere to host cells. In stage 2, microcolonies or "aggregates of cells" form and are stabilized by interacting with the surrounding host cells and ECM components, which may be cooperative with Scl1-Scl1 dimerization. The microcolonies proliferate producing a mature biofilm or "macrocommunity" that is often resistance to host immune defenses as well as environmental attacks. In stage 3, biofilm disruption occurs, which may be induced through proteolytic activities of secreted GAS proteases, nutrient deprivation, or other altered environmental conditions. Dissemination or dispersal of individual GAS cells, chains of cells, or loosely-associated microcolonies into adjacent tissues initiate the biofilm formation process again in a secondary niche. 


\section{References}

1. Akiyama, H., T. Hamada, W. K. Huh, O. Yamasaki, T. Oono, W. Fujimoto, and K. Iwatsuki. 2003. Confocal laser scanning microscopic observation of glycocalyx production by Staphylococcus aureus in skin lesions of bullous impetigo, atopic dermatitis and pemphigus foliaceus. Br J Dermatol 148:526-532.

2. Anthony, B. F., L. V. Perlman, and L. W. Wannamaker. 1967. Skin Infections And Acute Nephritis In American Indian Children. Pediatrics 39:263-279.

3. Berkower, C., M. Ravins, A. E. Moses, and E. Hanski. 1999. Expression of different group A streptococcal $M$ proteins in an isogenic background demonstrates diversity in adherence to and invasion of eukaryotic cells. Mol Microbiol 31:1463-1475.

4. Bessen, D. E., N. Kumar, G. S. Hall, D. R. Riley, F. Luo, S. Lizano, C. N. Ford, W. M. McShan, S. V. Nguyen, J. C. Dunning Hotopp, and H. Tettelin. 2011. Whole-genome association study on tissue tropism phenotypes in group A Streptococcus. J Bacteriol 193:6651-6663.

5. Bessen, D. E., and S. Lizano. 2010. Tissue tropisms in group A streptococcal infections. Future Microbiol 5:623-638.

6. Blackmore, T. K., V. A. Fischetti, T. A. Sadlon, H. M. Ward, and D. L. Gordon. 1998. M protein of the group A Streptococcus binds to the seventh short consensus repeat of human complement factor H. Infect Immun 66:1427-1431.

7. Bottas, A., and M. A. Richter. 2002. Pediatric autoimmune neuropsychiatric disorders associated with streptococcal infections (PANDAS). Pediatr Infect Dis J 21:67-71.

8. Brodsky, B., and J. Baum. 2008. Structural biology: Modelling collagen diseases. Nature 453:998-999.

9. Brodsky, B., G. Thiagarajan, B. Madhan, and K. Kar. 2008. Triple-helical peptides: an approach to collagen conformation, stability, and self-association. Biopolymers 89:345-353.

10. Carapetis, J., A. Steer, E. Mulholland, and M. Weber. 2005. The global burden of group A streptococcal diseases. Lancet Infect Dis 5:685-694.

11. Caswell, C., E. Lukomska, N. Seo, M. Höök, and S. Lukomski. 2007. Scl1dependent internalization of group A Streptococcus via direct interactions with the $\alpha_{2} \beta_{1}$ integrin enhances pathogen survival and re-emergence. Mol Microbiol 64:1319-1331.

12. Caswell, C. C., M. Barczyk, D. R. Keene, E. Lukomska, D. E. Gullberg, and S. Lukomski. 2008. Identification of the first prokaryotic collagen sequence motif that mediates binding to human collagen receptors, integrins $\alpha_{2} \beta_{1}$ and $\alpha_{11} \beta_{1}$. J Biol Chem 283:36168-36175.

13. Caswell, C. C., R. Han, K. M. Hovis, P. Ciborowski, D. R. Keene, R. T. Marconi, and S. Lukomski. 2008. The Scl1 protein of M6-type group A Streptococcus binds the human complement regulatory protein, factor $\mathrm{H}$, and inhibits the alternative pathway of complement. Mol Microbiol 67:584-596. 
14. Chen, S. M., Y. S. Tsai, C. M. Wu, S. K. Liao, L. C. Wu, C. S. Chang, Y. H. Liu, and P. J. Tsai. 2010. Streptococcal collagen-like surface protein 1 promotes adhesion to the respiratory epithelial cell. BMC Microbiol 10:320.

15. Cho, K., and M. Caparon. 2005. Patterns of virulence gene expression differ between biofilm and tissue communities of Streptococcus pyogenes. Mol Microbiol 57:1545-1556.

16. Cleary, P., Y. Matsuka, T. Huynh, H. Lam, and S. Olmsted. 2004. Immunization with C5a peptidase from either group A or B streptococci enhances clearance of group A streptococci from intranasally infected mice. Vaccine 22:4332-4341.

17. Cole, J. N., T. C. Barnett, V. Nizet, and M. J. Walker. 2011. Molecular insight into invasive group A streptococcal disease. Nat Rev Microbiol 9:724-736.

18. Conley, J., M. E. Olson, L. S. Cook, H. Ceri, V. Phan, and H. D. Davies. 2003. Biofilm formation by group a streptococci: is there a relationship with treatment failure? J Clin Microbiol 41:4043-4048.

19. Courtney, H. S., D. L. Hasty, and J. B. Dale. 2003. Serum opacity factor (SOF) of Streptococcus pyogenes evokes antibodies that opsonize homologous and heterologous SOF-positive serotypes of group A streptococci. Infect Immun 71:5097-5103.

20. Courtney, H. S., I. Ofek, T. Penfound, V. Nizet, M. A. Pence, B. Kreikemeyer, A. Podbielski, D. L. Hasty, and J. B. Dale. 2009. Relationship between expression of the family of $M$ proteins and lipoteichoic acid to hydrophobicity and biofilm formation in Streptococcus pyogenes. PLoS ONE 4:e4166.

21. Cunningham, M. W. 2000. Pathogenesis of group A streptococcal infections. Clin Microbiol Rev 13:470-511.

22. Davey, M. E., and A. G. O'Toole. 2000. Microbial biofilms: from ecology to molecular genetics. Microbiol Mol Biol Rev 64:847-867.

23. Dillon, H. C., C. W. Derrick, and M. S. Dillon. 1974. M-Antigens common to pyoderma and acute glomerulonephritis. J Infect Dis 130:257-267.

24. Dillon, H. C., and M. S. A. Dillon. 1974. New streptococcal serotypes causing pyoderma and acute glomerulonephritis Types 59, 60, and 61. Infect Immun 9:1070-1078.

25. Doern, C. D., R. C. Holder, and S. D. Reid. 2008. Point mutations within the streptococcal regulator of virulence (Srv) alter protein-DNA interactions and Srv function. Microbiology 154:1998-2007.

26. Doern, C. D., A. L. Roberts, W. Hong, J. Nelson, S. Lukomski, W. E. Swords, and S. D. Reid. 2009. Biofilm formation by group A Streptococcus: a role for the streptococcal regulator of virulence (Srv) and streptococcal cysteine protease (SpeB). Microbiology 155:46-52.

27. Donlan, R. M. 2011. Biofilm elimination on intravascular catheters: important considerations for the infectious disease practitioner. Clin Infect Dis 52:10381045.

28. Eckes, B., R. Nischt, and T. Krieg. 2010. Cell-matrix interactions in dermal repair and scarring. Fibrogenesis Tissue Repair 3:4. 
29. Efstratiou, A. 2000. Group A streptococci in the 1990s. J Antimicrob Chemother 45 Suppl:3-12.

30. Ffrench-Constant, C. 1995. Alternative splicing of fibronectin--many different proteins but few different functions. Exp. Cell Res. 221:261-271.

31. Fischetti, V. A. 1989. Streptococcal M protein: molecular design and biological behavior. Clin. Microbiol. Rev. 2:285-314.

32. Gilbert, P., J. Das, and I. Foley. 1997. Biofilm susceptibility to antimicrobials. Adv Dent Res 11:160-167.

33. Grenache, D. G., Z. Zhang, L. E. Wells, S. A. Santoro, J. M. Davidson, and M. M. Zutter. 2007. Wound healing in the $\alpha_{2} \beta_{1}$ integrin-deficient mouse: altered keratinocyte biology and dysregulated matrix metalloproteinase expression. J Invest Dermatol 127:455-466.

34. Grinnell, F., R. E. Billingham, and L. Burgess. 1981. Distribution of fibronectin during wound healing in vivo. J Invest Dermatol 76:181-189.

35. Grose, R., C. Hutter, W. Bloch, I. Thorey, F. M. Watt, R. Fassler, C. Brakebusch, and S. Werner. 2002. A crucial role of $\beta_{1}$ integrins for keratinocyte migration in vitro and during cutaneous wound repair. Development 129:23032315.

36. Gurjala, A. N., M. R. Geringer, A. K. Seth, S. J. Hong, M. S. Smeltzer, R. D. Galiano, K. P. Leung, and T. A. Mustoe. 2011. Development of a novel, highly quantitative in vivo model for the study of biofilm-impaired cutaneous wound healing. Wound Repair Regen 19:400-410.

37. Guzman, C. A., S. R. Talay, G. Molinari, E. Medina, and G. S. Chhatwal. 1999. Protective immune response against Streptococcus pyogenes in mice after intranasal vaccination with the fibronectin-binding protein SfbI. J Infect Dis 179:901-906.

38. Hall-Stoodley, L., J. W. Costerton, and P. Stoodley. 2004. Bacterial biofilms: from the natural environment to infectious diseases. Nat Rev Microbiol 2:95-108.

39. Han, R., C. C. Caswell, E. Lukomska, D. R. Keene, M. Pawlowski, J. M. Bujnicki, J. K. Kim, and S. Lukomski. 2006. Binding of the low-density lipoprotein by streptococcal collagen-like protein Scl1 of Streptococcus pyogenes. Mol Microbiol 61:351-367.

40. Han, R., A. Zwiefka, C. C. Caswell, Y. Xu, D. R. Keene, E. Lukomska, Z. Zhao, M. Höök, and S. Lukomski. 2006. Assessment of prokaryotic collagenlike sequences derived from streptococcal Scl1 and Scl2 proteins as a source of recombinant GXY polymers. Appl Microbiol Biotechnol 72:109-115.

41. Hanski, E., and M. Caparon. 1992. Protein F, a fibronectin-binding protein, is an adhesin of the group A Streptococcus Streptococcus pyogenes. PNAS 89:6172-6176.

42. Henningham, A., T. C. Barnett, P. G. Maamary, and M. J. Walker. 2012. Pathogenesis of group A streptococcal infections. Discov Med 13:329-342.

43. Henningham, A., C. M. Gillen, and M. J. Walker. 2012. Group A Streptococcal Vaccine Candidates: Potential for the Development of a Human Vaccine. Current topics in microbiology and immunology.

44. Hershberger, R. P., and L. A. Culp. 1990. Cell-type-specific expression of alternatively spliced human fibronectin IIICS mRNAs. Mol Cell Biol 10:662-671. 
45. Horstmann, R. D., H. J. Sievertsen, J. Knobloch, and V. A. Fischetti. 1988. Antiphagocytic activity of streptococcal $M$ protein: selective binding of complement control protein factor H. Proc Nat Acad Sci USA 85:1657-1661.

46. Humtsoe, J. O., J. K. Kim, Y. Xu, D. R. Keene, M. Höök, S. Lukomski, and K. K. Wary. 2005. A streptococcal collagen-like protein interacts with the $\alpha_{2} \beta_{1}$ integrin and induces intracellular signaling. J Biol Chem 280:13848-13857.

47. Hyland, K. A., B. Wang, and P. P. Cleary. 2007. Protein F1 and Streptococcus pyogenes resistance to phagocytosis. Infect Immun 75:3188-3191.

48. Ji, Y., B. Carlson, A. Kondagunta, and P. P. Cleary. 1997. Intranasal immunization with C5a peptidase prevents nasopharyngeal colonization of mice by the group A Streptococcus. Infect Immun 65:2080-2087.

49. Johnsson, E., K. Berggard, H. Kotarsky, J. Hellwage, P. F. Zipfel, U. Sjobring, and G. Lindahl. 1998. Role of the hypervariable region in streptococcal M proteins: binding of a human complement inhibitor. J. Immunol. 161:4894-4901.

50. Kania, R. E., G. E. Lamers, M. J. Vonk, P. T. Huy, P. S. Hiemstra, G. V. Bloemberg, and J. J. Grote. 2007. Demonstration of bacterial cells and glycocalyx in biofilms on human tonsils. Arch Otolaryngol Head Neck Surg 133:115-121.

51. Karlstrom, A., K. Jacobsson, M. Flock, J. Flock, and B. Guss. 2004. Identification of a novel collagen-like protein, SclC, in Streptococcus equi using signal sequence phage display. Vet Microbiol 104:179-188.

52. Kawabata, S., E. Kunitomo, Y. Terao, I. Nakagawa, K. Kikuchi, K. Totsuka, and S. Hamada. 2001. Systemic and mucosal immunizations with fibronectinbinding protein FBP54 induce protective immune responses against Streptococcus pyogenes challenge in mice. Infect Immun 69:924-930.

53. Kihlberg, B.-M., M. Collin, A. Olsen, and L. Björck. 1999. Protein H, an Antiphagocytic Surface Protein in Streptococcus pyogenes. Infect Immun 67:1708-1714.

54. Kimura, K. R., M. Nakata, T. Sumitomo, B. Kreikemeyer, A. Podbielski, Y. Terao, and S. Kawabata. 2012. Involvement of T6 pili in biofilm formation by serotype M6 Streptococcus pyogenes. J Bacteriol 194:804-812.

55. Knight, C. G., L. F. Morton, A. R. Peachey, D. S. Tuckwell, R. W. Farndale, and M. J. Barnes. 2000. The collagen-binding A-domains of integrins $\alpha_{2} \beta_{1}$ and $\alpha_{1} \beta_{1}$ recognize the same specific amino acid sequence, GFOGER, in native (triple-helical) collagens. J Biol Chem 275:35-40.

56. Kohan, M., A. F. Muro, E. S. White, and N. Berkman. 2010. EDA-containing cellular fibronectin induces fibroblast differentiation through binding to $\alpha_{4} \beta_{7}$ integrin receptor and MAPK/Erk 1/2-dependent signaling. FASEB J 24:45034512.

57. Kratovac, Z., A. Manoharan, F. Luo, S. Lizano, and D. E. Bessen. 2007. Population genetics and linkage analysis of loci within the FCT region of Streptococcus pyogenes J Bacteriol 189:1299-1310.

58. Kreikemeyer, B., M. Klenk, and A. Podbielski. 2004. The intracellular status of Streptococcus pyogenes: role of extracellular matrix-binding proteins and their regulation. Int J Med Microbiol 294:177-188. 
59. Lembke, C., A. Podbielski, C. Hidalgo-Grass, L. Jonas, E. Hanski, and B. Kreikemeyer. 2006. Characterization of biofilm formation by clinically relevant serotypes of group A streptococci. Appl Environ Microbiol 72:2864-2875.

60. Liao, Y. F., P. J. Gotwals, V. E. Koteliansky, D. Sheppard, and L. Van De Water. 2002. The EIIIA segment of fibronectin is a ligand for integrins $\alpha_{9} \beta_{1}$ and $\alpha_{4} \beta_{1}$ providing a novel mechanism for regulating cell adhesion by alternative splicing. J Biol Chem 277:14467-14474.

61. Liao, Y. F., K. G. Wieder, J. M. Classen, and L. Van De Water. 1999. Identification of two amino acids within the EIIIA (ED-A) segment of fibronectin constituting the epitope for two function-blocking monoclonal antibodies. J Biol Chem 274:17876-17884.

62. Liddington, R., and L. Bankston. 1998. The integrin I domain: crystals, metals and related artefacts. Structure 6:937-938.

63. Lukomski, S., K. Nakashima, I. Abdi, V. J. Cipriano, R. M. Ireland, S. D. Reid, G. G. Adams, and J. M. Musser. 2000. Identification and characterization of the scl gene encoding a group A Streptococcus extracellular protein virulence factor with similarity to human collagen. Infect Immun 68:6542-6553.

64. Lukomski, S., K. Nakashima, I. Abdi, V. J. Cipriano, B. J. Shelvin, E. A. Graviss, and J. M. Musser. 2001. Identification and characterization of a second extracellular collagen-like protein made by group A Streptococcus: control of production at the level of translation. Infect Immun 69:1729-1738.

65. Magnusson, M. K., and D. F. Mosher. 1998. Fibronectin: structure, assembly, and cardiovascular implications. Arterioscler Thromb Vasc Biol 18:1363-1370.

66. Manetti, A., C. Zingaretti, F. Falugi, S. Capo, M. Bombaci, F. Bagnoli, G. Gambellini, G. Bensi, M. Mora, A. Edwards, J. Musser, E. Graviss, J. Telford, G. Grandi, and I. Margarit. 2007. Streptococcus pyogenes pili promote pharyngeal cell adhesion and biofilm formation. Mol Microbiol 64:968983.

67. Martin, P. 1997. Wound healing--aiming for perfect skin regeneration. Science 276:75-81.

68. McNeil, S. A., S. A. Halperin, J. M. Langley, B. Smith, A. Warren, G. P. Sharratt, D. M. Baxendale, M. A. Reddish, M. C. Hu, S. D. Stroop, J. Linden, L. F. Fries, P. E. Vink, and J. B. Dale. 2005. Safety and immunogenicity of 26valent group A Streptococcus vaccine in healthy adult volunteers. Clin Infect Dis 41:1114-1122.

69. Mell, L. K., R. L. Davis, and D. Owens. 2005. Association between streptococcal infection and obsessive-compulsive disorder, Tourette's syndrome, and tic disorder. Pediatrics 116:56-60.

70. Midwood, K. S., L. V. Valenick, H. C. Hsia, and J. E. Schwarzbauer. 2004. Coregulation of fibronectin signaling and matrix contraction by tenascin-C and syndecan-4. Mol Biol Cell 15:5670-5677.

71. Mohs, A., T. Silva, T. Yoshida, R. Amin, S. Lukomski, M. Inouye, and B. Brodsky. 2007. Mechanism of stabilization of a bacterial collagen triple helix in the absence of hydroxyproline. J Biol Chem 282:29757-29765. 
72. Molinari, G., and G. S. Chhatwal. 1998. Invasion and survival of Streptococcus pyogenes in eukaryotic cells correlates with the source of the clinical isolates. $\mathbf{J}$ Infect Dis 177:1600-1607.

73. Monds, R. D., and G. A. O'Toole. 2009. The developmental model of microbial biofilms: ten years of a paradigm up for review. Trends Microbiol 17:73-87.

74. Moretti, G., M. Pasquini, G. Mandarelli, L. Tarsitani, and M. Biondi. 2008. What every psychiatrist should know about PANDAS: a review. Clin Pract Epidemol Ment Health 4:13.

75. Musser, J. M., and S. A. Shelburne, 3rd. 2009. A decade of molecular pathogenomic analysis of group A Streptococcus. J Clin Invest 119:2455-2463.

76. Nadell, C. D., J. B. Xavier, and K. R. Foster. 2009. The sociobiology of biofilms. FEMS Microbiol Rev 33:206-224.

77. Nerenberg, P. S., R. Salsas-Escat, and C. M. Stultz. 2008. Do collagenases unwind triple-helical collagen before peptide bond hydrolysis? Reinterpreting experimental observations with mathematical models. Proteins 70:1154-1161.

78. O'Loughlin, R., A. Roberson, P. Cieslak, R. Lynfield, K. Gershman, A. Craig, B. Albanese, M. Farley, N. Barrett, N. Spina, B. Beall, L. Harrison, A. Reingold, C. Van Beneden, and 2007. The epidemiology of invasive group A streptococcal infection and potential vaccine implications: United States, 20002004. Clin Infect Dis 45:853-862.

79. O'Toole, G., H. B. Kaplan, and R. Kolter. 2000. Biofilm formation as microbial development. Annu Rev Microbiol 54:49-79.

80. O'Toole, G. A. 2004. Microbiology: Jekyll or hide? Nature 432:680-681.

81. Oliver-Kozup, H. A., M. Elliott, B. A. Bachert, K. H. Martin, S. D. Reid, D. E. Schwegler-Berry, B. J. Green, and S. Lukomski. 2011. The streptococcal collagen-like protein-1 (Scl1) is a significant determinant for biofilm formation by group A Streptococcus. BMC Microbiol 11:262.

82. Olsen, R. J., and J. M. Musser. 2010. Molecular pathogenesis of necrotizing fasciitis. Annu Rev Pathol 5:1-31.

83. Ozeri, V., A. Tovi, I. Burstein, S. Natanson-Yaron, M. G. Caparon, K. M. Yamada, S. K. Akiyama, I. Vlodavsky, and E. Hanski. 1996. A two-domain mechanism for group A streptococcal adherence through protein $\mathrm{F}$ to the extracellular matrix. EMBO J 15:989-998.

84. Palmer, E. L., C. Ruegg, R. Ferrando, R. Pytela, and D. Sheppard. 1993. Sequence and tissue distribution of the integrin alpha 9 subunit, a novel partner of beta 1 that is widely distributed in epithelia and muscle. J Cell Biol 123:12891297.

85. Pandiripally, V., E. Gregory, and D. Cue. 2002. Acquisition of regulators of complement activation by Streptococcus pyogenes serotype M1. Infect Immun 70:6206-6214.

86. Pandiripally, V., L. Wei, C. Skerka, P. F. Zipfel, and D. Cue. 2003. Recruitment of complement factor $\mathrm{H}$-like protein 1 promotes intracellular invasion by group A streptococci. Infect Immun 71:7119-7128.

87. Pankov, R., and K. M. Yamada. 2002. Fibronectin at a glance. J Cell Sci 115:3861-3863. 
88. Paterson, G. K., L. Nieminen, J. M. Jefferies, and T. J. Mitchell. 2008. PclA, a pneumococcal collagen-like protein with selected strain distribution, contributes to adherence and invasion of host cells. FEMS Microbiol Lett 285:170-176.

89. Percival, S. L., C. Emanuel, K. F. Cutting, and D. W. Williams. 2012. Microbiology of the skin and the role of biofilms in infection. Int Wound J 9:1432.

90. Percival, S. L., K. E. Hill, D. W. Williams, S. J. Hooper, D. W. Thomas, and J. W. Costerton. 2012. A review of the scientific evidence for biofilms in wounds. Wound Repair Regen 20:647-657.

91. Peters, J. H., M. N. Grote, N. E. Lane, and R. J. Maunder. 2011. Changes in plasma fibronectin isoform levels predict distinct clinical outcomes in critically ill patients. Biomarker insights 6:59-68.

92. Potera, C. 1999. Forging a link between biofilms and disease. Science 283:18371839.

93. Proft, T., P. D. Webb, V. Handley, and J. D. Fraser. 2003. Two novel superantigens found in both group A and group C Streptococcus. Infect Immun 71:1361-1369.

94. Ramachandran, G. N. 1988. Stereochemistry of collagen. Int J Peptide Protein Res. 31:1-16.

95. Rasmussen, M., and L. Björck. 2001. Unique regulation of SclB-a novel collagen-like surface protein of Streptococcus pyogenes. Infect Immun 40:14271438.

96. Rasmussen, M., A. Eden, and L. Björck. 2000. SclA, a novel collagen-like surface protein of Streptococcus pyogenes. Infect Immun 68:6370-6377.

97. Rasmussen, M., M. Jacobsson, and L. Björck. 2003. Genome-based identification and analysis of collagen-related structural motifs in bacterial and viral proteins. J Biol Chem 278:32313-32316.

98. Rennard, S. I., G. W. Hunninghake, P. B. Bitterman, and R. G. Crystal. 1981. Production of fibronectin by the human alveolar macrophage: mechanism for the recruitment of fibroblasts to sites of tissue injury in interstitial lung diseases. Proc Natl Acad Sci U S A 78:7147-7151.

99. Reuter, M., C. C. Caswell, S. Lukomski, and P. F. Zipfel. 2010. Binding of the human complement regulators CFHR1 and factor $\mathrm{H}$ by streptococcal collagenlike protein 1 (Scl1) via their conserved $\mathrm{C}$ termini allows control of the complement cascade at multiple levels. J Biol Chem 285:38473-38485.

100. Rivera-Hernandez, T., J. Hartas, Y. Wu, Y. P. Chuan, L. H. Lua, M. Good, M. R. Batzloff, and A. P. Middelberg. 2013. Self-adjuvanting modular viruslike particles for mucosal vaccination against group A Streptococcus (GAS). Vaccine. 31:1950-1955.

101. Roberts, A. L., K. L. Connolly, C. D. Doern, R. C. Holder, and S. D. Reid. 2010. Loss of the group A Streptococcus regulator Srv decreases biofilm formation in vivo in an otitis media model of infection. Infect Immun 78:48004808.

102. Salsas-Escat, R., P. S. Nerenberg, and C. M. Stultz. 2010. Cleavage site specificity and conformational selection in type I collagen degradation. Biochemistry 49:4147-4158. 
103. Scales, B. S., and G. B. Huffnagle. 2013. The microbiome in wound repair and tissue fibrosis. J Pathol 229:323-331.

104. Schierle, C. F., M. De la Garza, T. A. Mustoe, and R. D. Galiano. 2009. Staphylococcal biofilms impair wound healing by delaying reepithelialization in a murine cutaneous wound model. Wound Repair Regen 17:354-359.

105. Schwarz-Linek, U., M. Höök, and J. R. Potts. 2006. Fibronectin-binding proteins of gram-positive cocci. Microbes Infect 8:2291-2298.

106. Schwarz-Linek, U., M. Höök, and J. R. Potts. 2004. The molecular basis of fibronectin-mediated bacterial adherence to host cells. Mol Microbiol 52:631-641.

107. Shinde, A. V., C. Bystroff, C. Wang, M. G. Vogelezang, P. A. Vincent, R. O. Hynes, and L. Van De Water. 2008. Identification of the peptide sequences within the EIIIA (EDA) segment of fibronectin that mediate integrin $\alpha_{9} \beta_{1^{-}}$ dependent cellular activities. J Biol Chem 283:2858-2870.

108. Singh, P., C. Chen, S. Pal-Ghosh, M. A. Stepp, D. Sheppard, and L. Van De Water. 2009. Loss of integrin $\alpha_{9} \beta_{1}$ results in defects in proliferation, causing poor re-epithelialization during cutaneous wound healing. J Invest Dermatol 129:217-228.

109. Singh, P., C. L. Reimer, J. H. Peters, M. A. Stepp, R. O. Hynes, and L. Van De Water. 2004. The spatial and temporal expression patterns of integrin $\alpha_{9} \beta_{1}$ and one of its ligands, the EIIIA segment of fibronectin, in cutaneous wound healing. J Invest Dermatol 123:1176-1181.

110. Skwarczynski, M., K. A. Kamaruzaman, S. Srinivasan, M. Zaman, I. C. Lin, M. R. Batzloff, M. F. Good, and I. Toth. 2012. An M-proteinderivedconformational peptide epitopevaccine candidate against Group A Streptococcus. Current drug delivery 10:39-45.

111. Steer, A. C., T. Lamagni, N. Curtis, and J. R. Carapetis. 2012. Invasive group a streptococcal disease: epidemiology, pathogenesis and management. Drugs 72:1213-1227.

112. Stevens, D. L. 1992. Invasive group A Streptococcus infections. Clin. Infect. Dis. 14:2-11.

113. Stewart, P. S., and M. J. Franklin. 2008. Physiological heterogeneity in biofilms. Nat Rev Microbiol 6:199-210.

114. Sutinen, M., T. Kainulainen, T. Hurskainen, E. Vesterlund, J. P. Alexander, C. M. Overall, T. Sorsa, and T. Salo. 1998. Expression of matrix metalloproteinases (MMP-1 and -2) and their inhibitors (TIMP-1, -2 and -3) in oral lichen planus, dysplasia, squamous cell carcinoma and lymph node metastasis. Br J Cancer 77:2239-2245.

115. Sylvestre, P., E. Couture-Tosi, and M. Mock. 2002. A collagen-like surface glycoprotein is a structural component of the Bacillus anthracis exosporium. Mol Microbiol 45:169-178.

116. Talay, S. R., P. Valentin-Weigand, P. G. Jerlstrom, K. N. Timmis, and G. S. Chhatwal. 1992. Fibronectin-binding protein of Streptococcus pyogenes: sequence of the binding domain involved in adherence of streptococci to epithelial cells. Infect Immun 60:3837-3844. 
117. Talay, S. R., P. Valentin-Weigand, K. N. Timmis, and G. S. Chhatwal. 1994. Domain structure and conserved epitopes of Sfb protein, the fibronectin-binding adhesin of Streptococcus pyogenes. Molecular Microbiology 13:531-539.

118. Talay, S. R., A. Zock, M. Rohde, G. Molinari, M. Oggioni, G. Pozzi, C. A. Guzman, and G. S. Chhatwal. 2000. Co-operative binding of human fibronectin to Sfbl protein triggers streptococcal invasion into respiratory epithelial cells. Cell Microbiol 2:521-535.

119. Taooka, Y., J. Chen, T. Yednock, and D. Sheppard. 1999. The integrin $\alpha_{9} \beta_{1}$ mediates adhesion to activated endothelial cells and transendothelial neutrophil migration through interaction with vascular cell adhesion molecule-1. J Cell Biol 145:413-420.

120. Tart, A., M. Walker, and J. Musser. 2007. New understanding of the group A Streptococcus pathogenesis cycle. Trends Microbiol 15:18-25.

121. To, W. S., and K. S. Midwood. 2011. Plasma and cellular fibronectin: distinct and independent functions during tissue repair. Fibrogenesis Tissue Repair 4:21.

122. Todd, S. J., A. J. G. Moir, M. J. Johnson, and A. Moir. 2003. Genes of Bacillus cereus and Bacillus anthracis encoding proteins of the exosporium. . Bacteriol 185:3373-3378.

123. Tognetti, L., C. Martinelli, S. Berti, J. Hercogova, T. Lotti, F. Leoncini, and S. Moretti. 2012. Bacterial skin and soft tissue infections: review of the epidemiology, microbiology, aetiopathogenesis and treatment: A collaboration between dermatologists and infectivologists. J Eur Acad Dermatol Venereol.

124. Top, F. H., Jr., L. W. Wannamaker, W. R. Maxted, and B. F. Anthony. 1967. $\mathrm{M}$ antigens among group A streptococci isolated from skin lesions. J Exp Med 126:667-685.

125. Webb, J. S., M. Givskov, and S. Kjelleberg. 2003. Bacterial biofilms: prokaryotic adventures in multicellularity. Curr Opin Microbiol 6:578-585.

126. Whatmore, A. M. 2001. Streptococcus pyogenes sclB encodes a putative hypervariable surface protein with a collagen-like repetitive structure. Microbiol 147:419-429.

127. Xu, C., Z. Yu, M. Inouye, B. Brodsky, and O. Mirochnitchenko. 2010. Expanding the family of collagen proteins: recombinant bacterial collagens of varying composition form triple-helices of similar stability. Biomacromolecules 11:348-356.

128. Xu, Y., D. R. Keene, J. M. Bujnicki, M. Höök, and S. Lukomski. 2002. Streptococcal Scl1 and Scl2 proteins form collagen-like triple helices. J Biol Chem 277:27312-27318.

129. Yamada, K. M. 2000. Fibronectin peptides in cell migration and wound repair. J Clin Invest 105:1507-1509.

130. Yamaguchi, M., Y. Terao, and S. Kawabata. 2012. Pleiotropic virulence factor - Streptococcus pyogenes fibronectin-binding proteins. Cell Microbiol 15:503511.

131. Yokasaki, Y., and D. Sheppard. 2000. Mapping of the cryptic integrin-binding site in osteopontin suggests a new mechanism by which thrombin can regulate inflammation and tissue repair. Trends Cardiovasc Med 10:155-159. 
132. Yoshizumi, A., J. M. Fletcher, Z. Yu, A. V. Persikov, G. J. Bartlett, A. L. Boyle, T. L. Vincent, D. N. Woolfson, and B. Brodsky. 2011. Designed coiled coils promote folding of a recombinant bacterial collagen. J Biol Chem 286:17512-17520.

133. Yu, Z., B. Brodsky, and M. Inouye. 2011. Dissecting a bacterial collagen domain from Streptococcus pyogenes: sequence and length-dependent variations in triple helix stability and folding. J Biol Chem 286:18960-18968.

134. Yu, Z., O. Mirochnitchenko, C. Xu, A. Yoshizumi, B. Brodsky, and M. Inouye. 2010. Noncollagenous region of the streptococcal collagen-like protein is a trimerization domain that supports refolding of adjacent homologous and heterologous collagenous domains. Protein Sci 19:775-785.

135. Yu, Z., R. Visse, M. Inouye, H. Nagase, and B. Brodsky. 2012. Defining requirements for collagenase cleavage in collagen type III using a bacterial collagen system. J Biol Chem 287:22988-22997.

136. Zabriskie, J. B., T. Poon-King, M. S. Blake, F. Michon, and M. Yoshinaga. 1997. Phagocytic, serological, and protective properties of streptococcal group A carbohydrate antibodies. Adv Exp Med Biol 418:917-919.

137. Zaman, M., A. B. Abdel-Aal, Y. Fujita, K. S. Phillipps, M. R. Batzloff, M. F. Good, and I. Toth. 2012. Immunological evaluation of lipopeptide group A Streptococcus (GAS) vaccine: structure-activity relationship. PLoS ONE 7:e30146.

138. Zaman, M., A. B. Abdel-Aal, Y. Fujita, Z. M. Ziora, M. R. Batzloff, M. F. Good, and I. Toth. 2012. Structure-activity relationship for the development of a self-adjuvanting mucosally active lipopeptide vaccine against Streptococcus pyogenes. J Med Chem 55:8515-8523. 


\section{West Virginia University \\ Health Sciences Center Graduate Programs Office Deficiency \& Shuttle Sheet Form}

TO:

Dr. Slawomir Lukomski

FROM: Lea Ann Defenbaugh, Health Sciences Graduate Programs Office, PO Box 9024

DATE: April 4, 2013

RE: Deficiencies and Shuttle Sheet Request Form (Necessary for Graduation)

\section{STUDENT'S NAME: Heaven A. Oliver-Kozup (700 925 774)}

The student named above has met all program specific requirements forgraduation fncluding publications.

SIGNATURE of GRADUATE PROGRAM DIRECTOR:

Qualifying Exams:

Preliminary:

(Or Assistant Dean if the director is the chair of the student's committee)

Research Proposal Defense:

Passed: $\mathbf{X}$

Date:

$7 / 18 / 08$

All conditions that must be met prior to the student's graduation are check-marked below.

Incomplete Grades for classes listed on attached sheet.

Transfer Credits Form (Student must see that Admissions \& Records (Downtown Campus), receives the original transcript).

Change of Status

Must be registered the semester he/she is to graduate

Complete "Application for Graduation and Diploma"

Submit Plan of Study

Submit Committee Approval form

Submit Qualifying Preliminary Exam form

Submit Research Proposal Defense form

Must have first-author manuscript, based on dissertation research, published or in-press (attach PMID and reprint or

PDF manuscript(s)

X_Submit updated CV prior to exit interview (pphillips@hsc.wvu.edu)

$\mathrm{X}$ - Complete the attached Alumni Sheet

X Schedule an EXIT interview with Dr. Minnear (contact Penny Phillips, pphillips@hsc.wvu.edu)

X Submit Electronic Dissertation or Thesis no later than Friday, May 3, 2013 at 5:00 PM

X _ Student submits completed ETD Doctoral or Master's Submission packet for final deadline date on

Friday, May 3, 2013 at 5:00 PM.

- Return within 24 hours of scheduled defense to Health Sciences Center Graduate Program's Office REGARDLESS of whether examination was held.

I participated in the final (X) Doctoral ( ) Thesis Defense for this student as scheduled and report my evaluation of the results as follows:

Committee's Signatures:

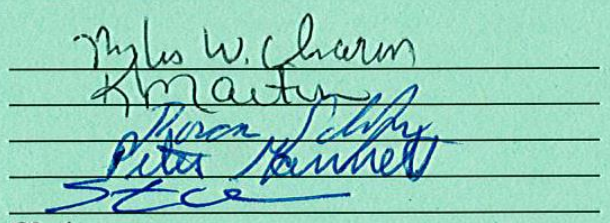

Chairperson
Names typed

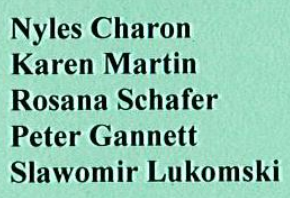

Passed Failed Date

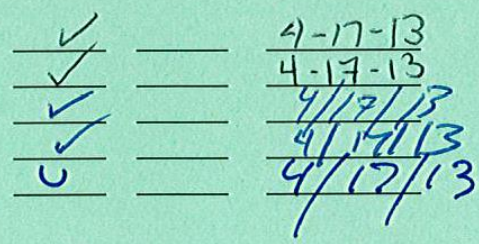

Degree and Major: PhD - Immunology and Microbial Pathogenesis

Dissertation Title: The Streptococcal Collagen-like Protein-1 (Scl1) is a Multifunctional Adhesin in Extracellular Matrix Interactions, Biofilm Formation, and Host Colonization by Group A Streptococcus. 